\title{
Growth and Properties of Topological Insulator Thin Films Based on the Bi2Se3 Compound
}

\author{
Sercan Babakiray
}

Follow this and additional works at: https://researchrepository.wvu.edu/etd

\section{Recommended Citation}

Babakiray, Sercan, "Growth and Properties of Topological Insulator Thin Films Based on the Bi2Se3 Compound" (2015). Graduate Theses, Dissertations, and Problem Reports. 5136.

https://researchrepository.wvu.edu/etd/5136

This Dissertation is protected by copyright and/or related rights. It has been brought to you by the The Research Repository @ WVU with permission from the rights-holder(s). You are free to use this Dissertation in any way that is permitted by the copyright and related rights legislation that applies to your use. For other uses you must obtain permission from the rights-holder(s) directly, unless additional rights are indicated by a Creative Commons license in the record and/ or on the work itself. This Dissertation has been accepted for inclusion in WVU Graduate Theses, Dissertations, and Problem Reports collection by an authorized administrator of The Research Repository @ WVU.

For more information, please contact researchrepository@mail.wvu.edu. 


\title{
Growth and Properties of Topological Insulator Thin Films Based on the $\mathrm{Bi}_{2} \mathrm{Se}_{3}$ Compound
}

\author{
Sercan Babakiray
}

\author{
Dissertation submitted \\ to the Eberly College of Arts and Sciences \\ at West Virginia University \\ in partial fulfillment of the requirements for the degree of \\ Doctor of Philosophy in \\ Physics
}

David Lederman, Ph.D., Chair

Pavel Borisov, Ph.D.

Xian-An Cao, Ph.D.

Yuri D. Glinka, Ph.D.

Tudor Stanescu, Ph.D.

Department of Physics and Astronomy

Morgantown, West Virginia

2015 


\title{
Abstract
}

\section{Growth and Properties of Topological Insulator Thin Films Based on the $\mathrm{Bi}_{2} \mathrm{Se}_{3}$ Compound}

\author{
Sercan Babakiray
}

This dissertation summarizes the growth, structural and electrical transport properties of topological insulators based on $\mathrm{Bi}_{2} \mathrm{Se}_{3}$ compound grown by molecular beam epitaxy. In the first three chapters, I review the theoretical background and experimental procedures necessary to understand the properties of topological insulators. The next three chapters cover the results of the structural characterization and electrical transport measurements. The final chapter summarizes the overall results and suggests future directions for research on $\mathrm{Bi}_{2} \mathrm{Se}_{3}$ compounds.

Topological insulators (TIs) are a new class of quantum matter with a bulk band gap and gapless metallic surface states [1-3]. The spin and momentum degrees of freedom are locked and are robust against non-magnetic perturbations. Here, I study the growth, structural and electronic properties of $\mathrm{Bi}_{2} \mathrm{Se}_{3}$ and $\mathrm{Mn}$ doped $\mathrm{Bi}_{2} \mathrm{Se}_{3}$ thin films.

$\mathrm{Bi}_{2} \mathrm{Se}_{3} / \mathrm{Bi}_{2-x} \mathrm{Mn}_{x} \mathrm{Se}_{3}$ thin films were grown on single crystal $\mathrm{Al}_{2} \mathrm{O}_{3}$ (0001) substrates by molecular beam epitaxy (MBE). Epitaxy was confirmed by reflection high energy electron diffraction (RHEED). Crystal orientation and lattice parameters were extracted from x-ray diffraction (XRD) measurements. The thin film thickness and roughness values were determined by fitting the x-ray reflectivity data (XRR) to a model based on optical scattering theory. To determine the Mn impurity site in $\mathrm{Bi}_{2-x} \mathrm{Mn}_{x} \mathrm{Se}_{3}$, extended x-ray absorption fine structure (EXAFS) measurements were performed at the Mn k-edge.

Finally, resistivity and Hall effect measurements were performed as functions of magnetic field and temperature. I focus on the weak antilocalization (WAL) and 2D magnetoconductance in the electrical transport measurements. 


\section{Contents}

Dedication ....................................... vii

Acknowledgments ..................... . . ix

List of Figures . . . . . . . . . . . . . . . . . . . xii

List of Tables . . . . . . . . . . . . . . . . . . xiii

1 Introduction 1

1.1 Introduction . . . . . . . . . . . . . . . . 1

1.1.1 Motivation .................... 1

1.1.2 Quantum Hall Effect (QHE) . . . . . . . . . . . . 2

1.1.3 Quantum Spin Hall Effect (QSHE) . . . . . . . . . . . . 3

1.1.4 Two Dimensional Topological Insulators . . . . . . . . . . . . 4

1.1.5 Three Dimensional Topological Insulators . . . . . . . . . 5

1.2 Theory of Topological Matter . . . . . . . . . . . . . . . 7

1.2.1 Berry Phase . . . . . . . . . . . . . . 7

1.2.2 The Dirac Equation . . . . . . . . . . . . . . . . . 9

1.2.3 Dirac Surface States . . . . . . . . . . . . . . . 13

1.2.4 Weak Antilocalization (WAL) . . . . . . . . . . 15

1.2.5 Electron-Electron Interaction (EEI) in Disordered Systems . . 18 
2 Growth and Characterization of $\mathrm{Bi}_{2} \mathrm{Se}_{3}$ and $\mathrm{Bi}_{2-x} \mathrm{Mn}_{x} \mathrm{Se}_{3}$ Thin Films 21

2.1 Thin Film Growth and Fabrication . . . . . . . . . . . . 21

2.1.1 Molecular Beam Epitaxy (MBE) _ . . . . . . . . . . . 21

2.1 .2 Photolithography . . . . . . . . . . . . . . . . 23

2.1 .3 Argon Ion Milling . . . . . . . . . . . . . . . . . . 26

2.2 Thin Film Characterization Techniques . . . . . . . . . . . 26

2.2.1 Reflection High Energy Electron Diffraction (RHEED) . . . 26

$2.2 .2 \quad$ X-ray Diffraction $(\mathrm{XRD}) \ldots \ldots \ldots \ldots$

$2.2 .3 \quad$ X-ray Reflectivity $(\mathrm{XRR}) \ldots \ldots \ldots \ldots \ldots$

2.2.4 Atomic Force Microscopy $(\mathrm{AFM}) \quad \ldots \ldots \ldots$. . . . . . . 31

2.2.5 X-ray Photoelectron Spectroscopy (XPS) . . . . . . . . . 33

2.2.6 Extended X-ray Absorption Fine Structure (EXAFS) . . . . . 35

$2.2 .7 \quad$ Hall Effect $\ldots \ldots \ldots \ldots \ldots \ldots$

3 Experimental Methods $\quad 40$

3.1 Sample Preparation and MBE Growth . . . . . . . . . . . 40

3.2 Structural Characterization $\ldots \ldots \ldots \ldots \ldots$. . . . . . . . 41

3.2.1 Reflection High Energy Electron Diffraction (RHEED) . . . . 41

3.2 .2 X-ray Diffraction (XRD) $\ldots \ldots \ldots \ldots \ldots \ldots$

3.2 .3 X-ray Reflectivity $(\mathrm{XRR}) \ldots \ldots \ldots \ldots$. . . . . . . . . 44

3.2.4 Atomic Force Microscopy (AFM) $\ldots \ldots \ldots \ldots \ldots$

3.2.5 X-ray Photoelectron Spectroscopy (XPS) . . . . . . . . 47

3.2.6 Extended X-ray Absorption Fine Structure (EXAFS) . . . . 47

3.3 Electrical Transport . . . . . . . . . . . . . . . . . . . . . 48 
4 Structural Properties of $\mathrm{Bi}_{2-x} \mathrm{Mn}_{x} \mathrm{Se}_{3}$ Thin Films Grown Via Molecular Beam Epitaxy $\quad 50$

4.1 Introduction . . . . . . . . . . . . . . . 50

4.2 Growth and Structural Characterization . . . . . . . . . . 51

$4.2 .1 \quad$ XPS Results . . . . . . . . . . . . . . . . . . . 56

$4.2 .2 \quad$ EXAFS Analysis . . . . . . . . . . . . . . . . . . 59

4.3 Electrical Characterization . . . . . . . . . . . . . . . . . . 65

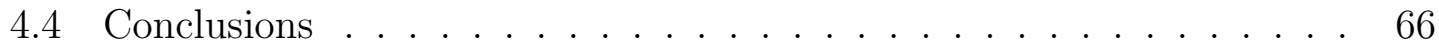

5 Single vs. Three Channel HLN Model for $\mathrm{Bi}_{2-x} \mathrm{Mn}_{x} \mathrm{Se}_{3}$ Thin Films

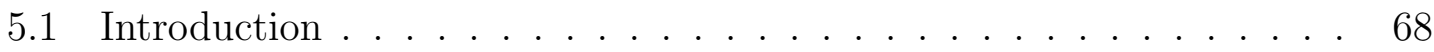

5.2 Growth and Structural Characterization _ . . . . . . . . . . 70

5.3 Carrier Density and Hall Mobility . . . . . . . . . . . . . . 72

5.4 Single Channel HLN Fit Results for 12 QL $\mathrm{Bi}_{2-x} \mathrm{Mn}_{x} \mathrm{Se}_{3}$ Thin Films . 77

$5.4 .1 \alpha$ and Number of Channels . . . . . . . . . . . . 77

5.4.2 Phase Coherence Length and $\alpha$ Coefficient . . . . . . . . 79

5.4.3 Temperature Dependence of $\alpha$ and Channel Separation . . . . 81

5.4.4 Temperature Dependence of $L_{\phi}$ and Decay Mechanism . . . 83

5.5 Three Channel HLN Fit Results for $12 \mathrm{QL} \mathrm{Bi}_{2-x} \mathrm{Mn}_{x} \mathrm{Se}_{3}$ Thin Films . 85

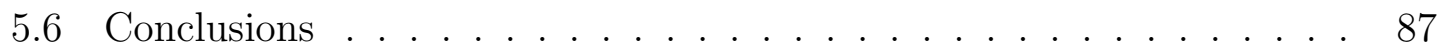

6 Low Temperature Transport Properties of $\mathrm{Bi}_{2} \mathrm{Se}_{3}$ Thin Films 88

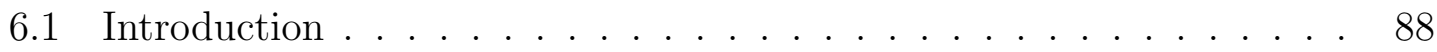

6.2 Sample Preparation and Experimental Methods . . . . . . . . . . . 89

6.3 HLN Formalism of Perpendicular Magnetoconductance . . . . . . . . 92 
6.4 Altshuler-Aronov Formalism of Parallel Magnetoconductance . . . . . 101

6.5 Temperature Dependence of Conductivity and Electron-Electron Interaction ......................... . . . 104

6.6 Summary and Conclusions . . . . . . . . . . . . . . . . 110

\section{Summary}

113

Bibliography

125 


\section{Dedication}

Dedicated to my mother, Narguzel Babakiray. I feel grateful for all the countless sacrifices she made for me and my brothers. 


\section{Acknowledgments}

This thesis becomes a reality with the kind support and help of many individuals. I would like to extend my sincere thanks to all of them.

First and foremost, I would like to express my special gratitude and thanks to my adviser. Prof. David Lederman expertly guided me through my graduate education. His enthusiasm for physics and research kept me constantly engaged with my work. Without his help and guidance this project would not be completed. His experience in material science, thin film research and vacuum technology were the key factors for my achievements at West Virginia University.

I am highly indebted to Dr. Yuri D. Glinka for measuring and analyzing the optical properties of our $\mathrm{Bi}_{2} \mathrm{Se}_{3}$ thin films. Without his hard work, an important part of this research would not be completed. His expertise in optics helped us to discuss main properties of surface states in topological insulators from optical point of view.

I also thank Dr. Alan Bristow and Derek Bas for measuring the injection currents in our $\mathrm{Bi}_{2} \mathrm{Se}_{3}$ thin films.

Special thanks to my committee members; Dr. Pavel Borisov, Dr. Xian-An Cao and Dr. Tudor Stanescu for accepting to be a part of my thesis committee.

An extra thank to Dr. Pavel Borisov. Dr. Borisov's mentoring and our technical discussions during weekly group meetings have been especially valuable for me. 
I would like to thank Dr. Matthew A. Marcus, Dr. Kartik Tarafder, Dr. Mikel Holcomb, and Dr. F. Bridges for taking and interpreting the EXAFS data at Advanced Light Source, Lawrence Berkeley National Laboratory. EXAFS was the key measurement in determining local structure in $\mathrm{Bi}_{2-x} \mathrm{Mn}_{x} \mathrm{Se}_{3}$ thin films.

In addition, thanks also goes to my coworkers, Trent Johnson, Toyanath Joshi, Amit KC, Rachel Henderson, Harley Hart and Dr. Wei Ding. I always felt very lucky working with a great team of people. I appreciate the way they helped me with my research.

My appreciation extends to West Virginia University Physics and Astronomy Department and especially to Sherry Puskar for her patience and constant help.

I am privileged to have friends from Turkey with whom I crossed paths with in Morgantown, WV. It was hardly possible for me to thrive in my doctoral work without the precious support of these personalities. I especially thank Ozcan and Berk for keeping me company during the final phase of writing my thesis.

Above all, I would like to thank my wife, my companion Seda. Your patience and calmness during ups and downs of my research always put be back on track. I can not imagine a more special soul-mate, thanks for being with me. Thank you for your constant love and support. But most importantly, thank you for believing in me.

This project would not be completed without the financial support from funding agencies. This work was supported by a Research Challenge Grant from the West Virginia Higher Education Policy Commission at WVU. Work at the ALS and the LBL was supported by the US Department of Energy. 


\section{List of Figures}

1.1 Cartoon depicting the $\mathrm{QHE} \ldots \ldots . \ldots . \ldots 2$

1.2 Illustration of QHE and QSHE . . . . . . . . . . . . . 3

1.3 Band inversion in HgTe quantum wells . . . . . . . . . . . . . 4

1.4 Dirac surface states in $\mathrm{Bi}_{2} \mathrm{Te}_{3} \ldots \ldots \ldots \ldots$

1.5 Spin orientation in momentum space . . . . . . . . . . . . . 12

1.6 Backscattering and localization effects . . . . . . . . . . 16

2.1 Picture of a typical MBE system . . . . . . . . . . . . . . . 23

2.2 Photolithography with positive and negative photoresists . . . . . 25

2.3 Typical RHEED setup . . . . . . . . . . . . . . . . 27

2.4 RHEED oscillations for GaAs . . . . . . . . . . . . . . . 28

2.5 Standard x-ray diffractometer . . . . . . . . . . . . . . . 30

2.6 Schematics for x-ray diffraction . . . . . . . . . . . . 31

2.7 Geometrical illustration of diffraction conditions . . . . . . . . . . . 32

2.8 AFM schematics . . . . . . . . . . . . . . . . . 33

2.9 Typical XPS schematics . . . . . . . . . . . . . . . . 34

2.10 Absorption edges in EXAFS . . . . . . . . . . . . . 36

2.11 EXAFS spectra . . . . . . . . . . . . . . . 36

2.12 Typical geometry in a Hall experiment . . . . . . . . . . . . . . . . . 39 


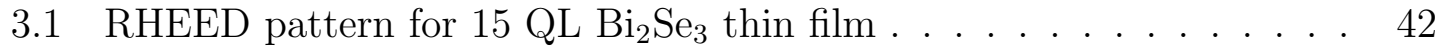

3.2 XRD pattern for $15 \mathrm{QL} \mathrm{Bi}_{2} \mathrm{Se}_{3}$ thin film . . . . . . . . . 43

3.3 Cartoon representing the XRR fit models . . . . . . . . . . . . 45

3.4 XRR data for $15 \mathrm{QL} \mathrm{Bi}_{2} \mathrm{Se}_{3}$ film . . . . . . . . . . . . . 46

3.5 AFM image of a typical $\mathrm{Bi}_{2} \mathrm{Se}_{3}$ film after $\mathrm{MgF}_{2}$ capping . . . . . . 46

3.6 Six contact Hall bar used in transport measurements . . . . . . . . . 49

4.1 RHEED patterns for $30 \mathrm{QL} \mathrm{Bi}_{2-x} \mathrm{Mn}_{x} \mathrm{Se}_{3}$ thin films . . . . . . . 52

4.2 AFM images of $30 \mathrm{QL} \mathrm{Bi}_{2-x} \mathrm{Mn}_{x} \mathrm{Se}_{3}$ thin films . . . . . . . . 53

4.3 Low angle x-ray reflectivity (XRR) data for $30 \mathrm{QL} \mathrm{Bi}_{2-x} \mathrm{Mn}_{x} \mathrm{Se}_{3}$ thin

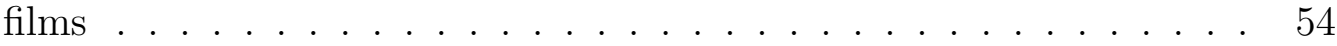

4.4 Out of plane XRD data for $\mathrm{Bi}_{2-x} \mathrm{Mn}_{x} \mathrm{Se}_{3}$ thin films $\ldots \ldots \ldots . . \quad 55$

4.5 Rocking curves for $\mathrm{Bi}_{2-x} \mathrm{Mn}_{x} \mathrm{Se}_{3}$ thin films $\ldots \ldots \ldots \ldots$

4.6 XPS data for a Mn-doped $\mathrm{Bi}_{2} \mathrm{Se}_{3}$ film . . . . . . . . . . . 57

4.7 EXAFS data for a Mn-doped $\mathrm{Bi}_{2} \mathrm{Se}_{3}$ thin film . . . . . . . . . 61

4.8 Unit cell representing a $\mathrm{Mn}$ doped $\mathrm{Bi}_{2} \mathrm{Se}_{3}$ thin film . . . . . . . . 62

4.9 Resistivity and Hall effect results for $30 \mathrm{QL} \mathrm{Bi}_{2-x} \mathrm{Mn}_{x} \mathrm{Se}_{3}$ thin films . 65

5.1 XRD data for $\mathrm{Bi}_{2} \mathrm{Se}_{3}$ and $\mathrm{Bi}_{2-x} \mathrm{Mn}_{x} \mathrm{Se}_{3}$ films . . . . . . . . 70

5.2 XRR data and fit for $12 \mathrm{QL} \mathrm{Bi}_{2-x} \mathrm{Mn}_{x} \mathrm{Se}_{3}$ films $\ldots \ldots \ldots \ldots . \ldots 71$

5.3 Hall effect data for $\mathrm{Bi}_{2} \mathrm{Se}_{3}$ thin films. . . . . . . . . . . . . . 72

5.4 Hall effect data for $12 \mathrm{QL} \mathrm{Bi}_{2-x} \mathrm{Mn}_{x} \mathrm{Se}_{3}$ thin films . . . . . . . . 74

5.5 HLN formula results for $12 \mathrm{QL} \mathrm{Bi}_{2-x} \mathrm{Mn}_{x} \mathrm{Se}_{3}$ thin films $\ldots \ldots \ldots 80$

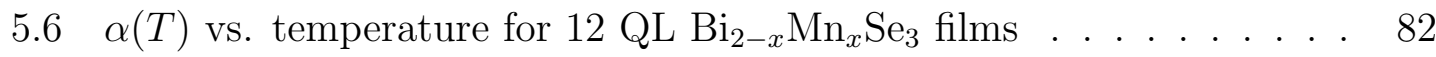

$5.7 L_{\phi}$ vs. temperature for $12 \mathrm{QL} \mathrm{Bi}_{2-x} \mathrm{Mn}_{x} \mathrm{Se}_{3}$ films $\ldots \ldots \ldots . . . \quad 84$

5.8 Three channel HLN fit results for $12 \mathrm{QL} \mathrm{Bi}_{2-x} \mathrm{Mn}_{x} \mathrm{Se}_{3}$ films . . . . . 86 
6.1 RHEED data for $12-25 \mathrm{QLs} \mathrm{Bi}_{2} \mathrm{Se}_{3}$ films $\ldots \ldots \ldots \ldots \ldots$

6.2 RHEED oscillations for 12-25 $\mathrm{QLs} \mathrm{Bi}_{2} \mathrm{Se}_{3}$ films . . . . . . . . . . . 91

6.3 Characterization data for 12-25 QLs $\mathrm{Bi}_{2} \mathrm{Se}_{3}$ films . . . . . . . . . . 93

6.4 Magnetoconductance data and fits for 12-25 $\mathrm{QLs} \mathrm{Bi}_{2} \mathrm{Se}_{3}$ films based on three channels transport model . . . . . . . . . . . . . . . 94

6.5 Sandwich structure of $\mathrm{Bi}_{2-x} \mathrm{Mn}_{x} \mathrm{Se}_{3}$ films with $x=0.026 \ldots \ldots .97$

$6.6 L_{\phi}$ vs. temperature for $12-25 \mathrm{QLs} \mathrm{Bi}_{2} \mathrm{Se}_{3}$ films . . . . . . . . 100

6.7 Parallel MC data for $12-25 \mathrm{QLs}^{\mathrm{Bi}} \mathrm{Se}_{3}$ films . . . . . . . . . . 105

6.8 Temperature dependence of conductivity for 12-25 $\mathrm{QLs} \mathrm{Bi}_{2} \mathrm{Se}_{3}$ films . 108

6.9 Change in screening parameter $\Delta \widetilde{F}_{b}$ vs. thickness for 12-25 $\mathrm{QLs} \mathrm{Bi}_{2} \mathrm{Se}_{3}$

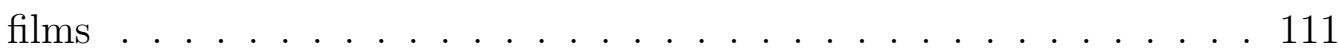




\section{List of Tables}

2.1 Steps in photolithography process . . . . . . . . . . . . 24

4.1 XPS measurement results for $12 \mathrm{QL} \mathrm{Bi}_{2-x} \mathrm{Mn}_{x} \mathrm{Se}_{3}$ film with $x=0.13 \quad 59$

4.2 EXAFS fit results for $30 \mathrm{QL} \mathrm{Bi}_{2-x} \mathrm{Mn}_{x} \mathrm{Se}_{3}$ film with $x=0.063 \ldots 63$

6.1 XRR fit results for 12-25 $\mathrm{QLs}^{\mathrm{Bi}} \mathrm{Bi}_{2} \mathrm{Se}_{3}$ thin films . . . . . . . . . 95

6.2 Typical semiconductor transport parameters at $T=2 \mathrm{~K} \ldots \ldots . \quad 99$ 


\section{Chapter 1}

\section{Introduction}

\section{$1.1 \quad$ Introduction}

\subsubsection{Motivation}

The existence of TI surface states was first recognized theoretically and predicted to appear in mercury telluride, bismuth antimony, bismuth selenide and bismuth telluride compounds $[1,4-7]$. The surface states of a topological insulator are spinpolarized and robust against non-magnetic perturbations [8]. This unique property makes TIs attractive candidates for solid state and spintronic devices. Also, it has been predicted that TIs can display exotic physics such as quantum anomalous Hall

effect [9], Majorana fermions [10] and unusual magneto-optical Kerr and Faraday effects [11], when the time reversal symmetry is broken due to magnetic impurities. Impurity doping tends to deteriorate the crystal structure, however, which causes the Fermi level to lie within the conduction band of the surface states. This in turn makes the interpretation of transport data difficult, because conduction occurs both in the surface states and bulk conduction bands. Therefore, the motivation 


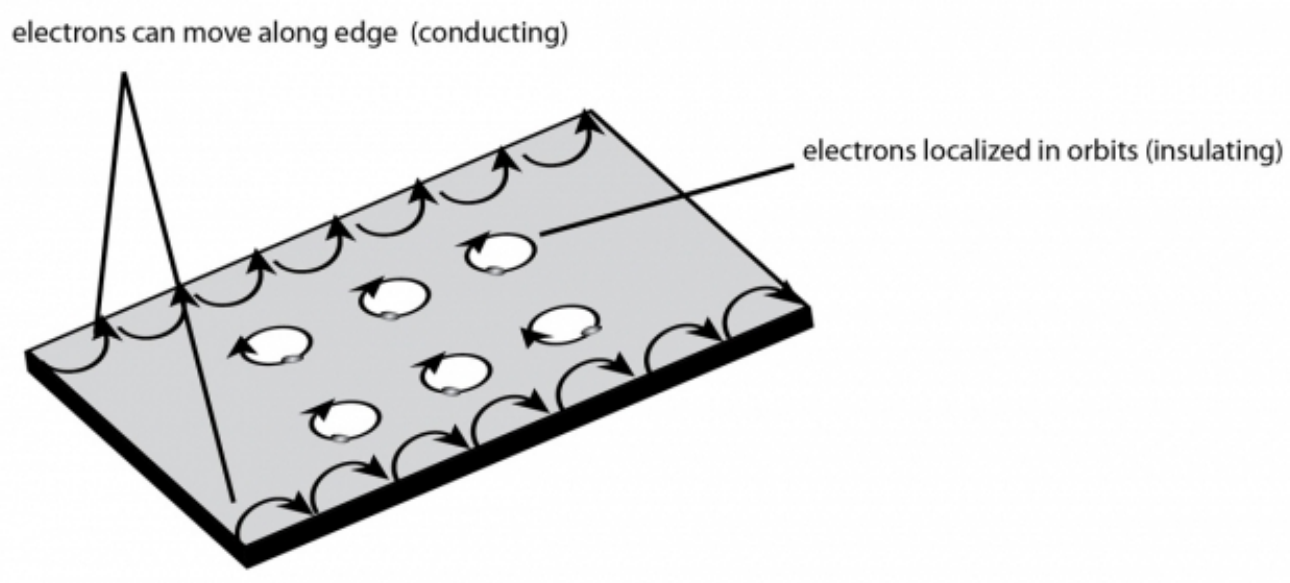

Figure 1.1: Cartoon depicting the QHE with the edge states on the boundary and the insulating states in the bulk of the material. From reference [12].

behind this work is to understand the relation between the structural and electronic transport properties of topological insulators.

\subsubsection{Quantum Hall Effect (QHE)}

Prior to 1980, all condensed matter were classified by spontaneous symmetry breaking. In 1980, Klitzing et al. discovered new quantum state of matter that only depends on the topology of the surface [13]. In a two dimensional material that is subject to a strong magnetic field, electrons move in circular cyclotron orbits associated with the Lorentz force. On the edge of the sample, electrons skip to complete these orbits, thus creating a one dimensional current channel. The states located towards the middle of such a material do not sustain current flow, and they thus behave as an insulator as shown in Fig. 1.1. As a result, the Hall conductance is quantized with rational multiples of $e^{2} / h[13]$. The quantization of Hall conductance originates from Landau quantization of a 2D electron gas. The application of mag- 


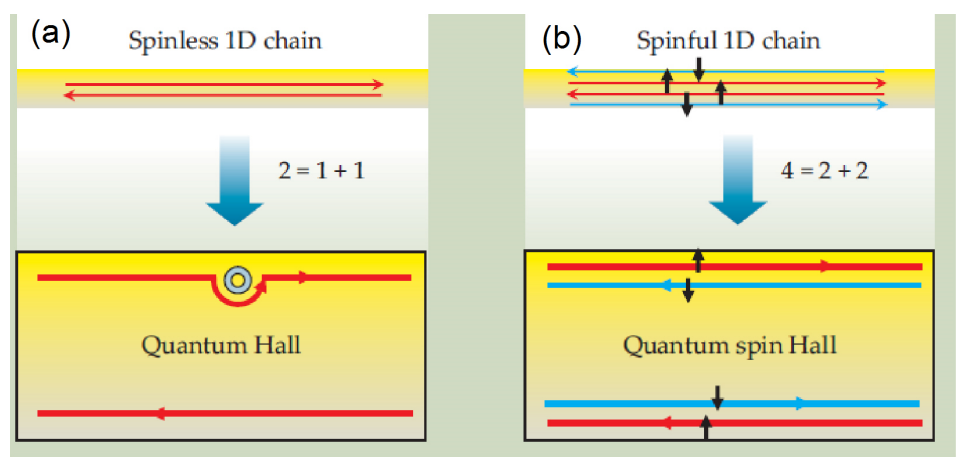

Figure 1.2: (a) Cartoon illustrating the spinless QHE. The numbers $2=1+1$ corresponds to the two degrees of freedom; a forward mover and a backward mover. (b) QSHE with spinful 1D chain of electrons. There are four basic channels which are spatially separated in a QSH bar. $4=2+2$ represents the movers at upper edge containing a forward mover with spin up and a backward mover with spin down, for the lower edge state. This figure applies for two-dimensional (2D) transport that has separate channels. The top part of the figure is $1 \mathrm{D}$, the bottom is part $2 \mathrm{D}$. From reference [8].

netic field shifts the energy of quantized Landau levels (LLs) up and down which cross the Fermi level at exact discrete numbers corresponding to the edge states. Whenever a crossing occurs, a plateau appears in the Hall conductance. The beauty of the QHE is that it is independent of sample dimensions and preparation process.

\subsubsection{Quantum Spin Hall Effect (QSHE)}

It has been predicted that QHE can occur in 2D systems without the application of an extrinsic magnetic field [14-16]. In essence, the spin-orbit coupling creates an intrinsic magnetic field that couples to the spin of electrons. This momentumdirection dependent coupling defines the motion of electrons in a unique fashion. Electrons in two edge channels move with opposite momentum. The difference between QHE and QSHE is shown in Fig. 1.2. For the QHE states, symbolic equation $2=1+1$ illustrates the the electrons moving in forward and backward directions on opposite sides of the Hall bar, resulting in two degrees of freedom, in total. These 


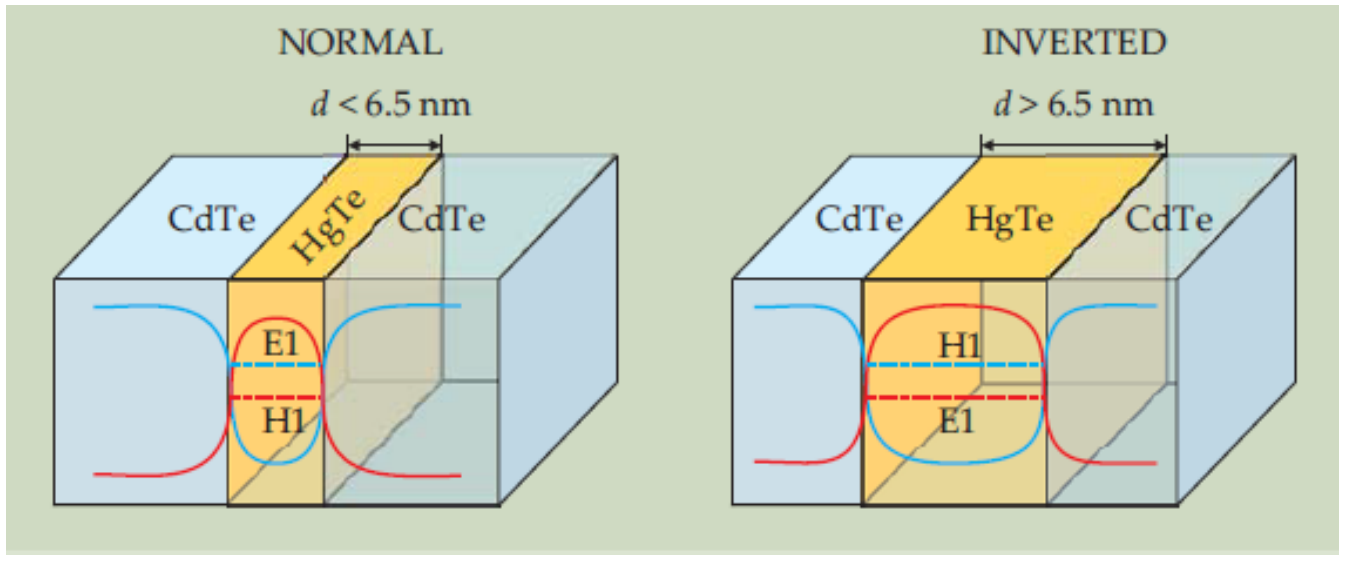

Figure 1.3: HgTe quantum well structure. Blue curve represents the potential energy well for electrons in conduction band and red curve is the barrier for holes in valence band. Both electrons and holes are free to move within CdTe layers but they are confined in the HgTe quantum well. E1 and $\mathrm{H} 1$ represent the conduction band minimum and valence band maximum respectively. When the layer thickness of quantum well $d \leq 6.5 \mathrm{~nm}$, the bands look like a regular insulator. On the other hand when $d>6.5 \mathrm{~nm}$, the bands are inverted (on the right) and resemble a QSH insulator. Adapted from reference [8].

QHE spinless states do not have time-reversal symmetry. Because the electrons in QHE forward and backward channels travel in opposite directions, backscattering is forbidden. Therefore, these states are robust and electrons go around impurities without scattering. On the other hand, QSHE is the superposition of two QHE systems in which electrons in edge states move in opposite direction creating a net forward spin flow at the top edge and backward spin flow at the bottom. Consequently, the equation $4=2+2$ demonstrates the four degrees of freedom in the system.

\subsubsection{Two Dimensional Topological Insulators}

In typical semiconductors, the conduction band is separated from the valence band by an energy gap. However, in systems with strong spin-orbit coupling, the 
p-orbital band is pushed above the s-orbital band, causing the so-called "band inversion". Bernevig, Hughes and Zhang proposed a mechanism to search for topological protection in two dimensional quantum wells (QWs) of mercury telluride. They modelled a semiconductor heterojunction which sandwich a mercury telluride layer (HgTe) in between two cadmium telluride ( $\mathrm{CdTe}$ ) layers [4]. When the thickness of $\mathrm{HgTe}$ layer is below $6.5 \mathrm{~nm}$, the structure is a trivial insulator with a positive energy gap. If the HgTe layer thickness is above $6.5 \mathrm{~nm}$, strong spin orbit coupling inverts the conduction and valence bands (Fig. 1.3), and the system behaves like a QSH insulator. Soon after these theoretical predictions were made, experimental verification of QSHE was achieved by Molenkamp's group [5] on HgTe heterostructures grown via molecular beam epitaxy (MBE). They measured the conductance as a function of gate voltage and tuned the electrical conductivity from $n$ - to $p$ - type, passing through the insulating phase. For a typical trivial insulator, there is zero conductance inside the band gap. However for a QSH insulator the conductance for a pair of edge states is equal to $2 e^{2} / h$, with a contribution of $e^{2} / h$ for each channel. For thin QWs, the insulating phase was detected with extremely small conductance values at low temperatures. However, the thicker QW samples showed a quantized value of conductance very close to $2 e^{2} / h$. Also, the variation of the width of the samples did not change this quantized value of conductance, proving that the effect must be associated with the edge states.

\subsubsection{Three Dimensional Topological Insulators}

Fu and Kane predicted that bismuth based semiconducting alloys such as $\mathrm{Bi}_{1-x} \mathrm{Sb}_{x}$ can be three dimensional (3D) topological insulators due to presence of strong spinorbit coupling (SOC) in bismuth element [1]. Similar to the the presence of spin po- 

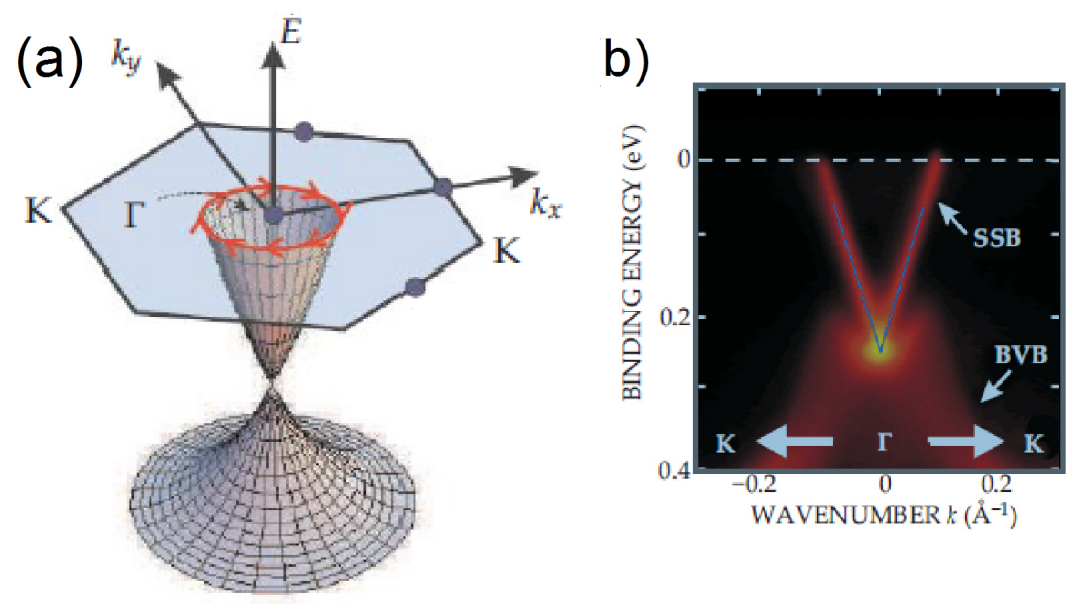

Figure 1.4: (a) Spin-momentum locking on the surface states of a 3D topological insulator (b) ARPES data showing the dispersion relation for a pure $\mathrm{Bi}_{2} \mathrm{Te}_{3}$ sample. Linar dispersion relation of surface state bands (SSB) appears above the parabolic dispersion of bulk valence bands (BVB). Dashed line is the Fermi level and blue lines crosses at the tip of the cone (Dirac point). From reference [8]

larized current in the 1D edge channels in a QSH insulator, a 3D topological insulator has spin polarized current on its surfaces. The strong SOC results in a Hamiltonian which is formally identical to the relativistic Dirac equation which gives a linear dispersion relation for surface states. The dispersion of the surface states forms a Dirac cone with its origin at $k=0$ and the surface states are protected by time reversal symmetry (TRS). The experimental observation of the Dirac surface states was first achieved using angle-resolved photoemission spectroscopy (ARPES) measurements performed on $\mathrm{Bi}_{1-x} \mathrm{Sb}_{x}$ by Hasan's group at Princeton [6]. Despite the observation of Dirac-like linear dispersion relations, the surface states still were mixed with bulk states in $\mathrm{Bi}_{1-x} \mathrm{Sb}_{x}$. Later on, search for a wider band gap 3D topological insulators ended with the discovery of second generation of materials such as $\mathrm{Bi}_{2} \mathrm{Te}_{3}$ and $\mathrm{Bi}_{2} \mathrm{Se}_{3}$ which are also known as excellent thermoelectric materials [17,18]. Spin-momentum locking of surface states was confirmed by using spin-integrated angle-resolved pho- 
toemission spectroscopy (SARPES) [19] as shown in Fig. 1.4a. Pure Dirac states were first observed in 3D $\left(\mathrm{Bi}_{1-x} \mathrm{Sn}_{x}\right)_{2} \mathrm{Te}_{3}$ where $x=0.67$ [20], with the data shown in the Fig. 1.4b. The formation of a single Dirac cone with a linear dispersion was realized for the massless fermions. The parabolic dispersion of bulk band electrons were also confirmed via ARPES measurements performed on these $\left(\mathrm{Bi}_{1-x} \mathrm{Sn}_{x}\right)_{2} \mathrm{Te}_{3}$ single crystals.

\subsection{Theory of Topological Matter}

The energy eigenstates $E_{n}(k)$ of a system with periodic potential are given by solutions of Schrodinger equation. Bloch functions $u_{n}(k)$ which are the solution to the Bloch Hamiltonian $H(k)$ extend over the Brillouin zone with a crystal momentum $k$. The band structure and the dispersion are well defined in terms of this crystal momentum.

\subsubsection{Berry Phase}

Classification of topological matter is related to the topological band theory and can be understood in terms of the Berry Phase [21]. If a system is adiabatically allowed to vary around a closed path, the Bloch wavefunctions $u_{n}(k)$ pick up a geometric phase called the Berry Phase. The time evolution of these Bloch wavefunctions is given by the time-dependent Schrodinger equation

$$
H\left(k(t)\left|u_{n}\left(k_{0}\right), t_{0} ; t>=i \hbar \frac{\partial}{\partial t}\right| u_{n}\left(k_{0}\right), t_{0} ; t>,\right.
$$


where the quantum mechanical expression of time dependent Bloch state is given by $[22]$

$$
\left|u_{n}\left(k_{0}\right), t_{0} ; t>=\exp \left[-\frac{i}{\hbar} \int_{0}^{t} E_{n}\left(k\left(t^{\prime}\right) d t^{\prime}\right)\right] \exp \left(i \gamma_{n}(t)\right)\right| u_{n}(k(t)>.
$$

The two terms on the right side of the equation correspond to dynamical and geometric phase factors, respectively. After substituting Eq. (1.2) into Eq. (1.1), one obtains the geometric phase

$$
\gamma_{n}(t)=i<u_{n}(k(t)) \mid \nabla_{k} u_{n}(k(t))>\frac{d}{d t} k(t) .
$$

In the parameter space, this can be expressed as a path integral

$$
\gamma_{n}(t)=\int_{C} d k \cdot A_{n}(k)
$$

where $A_{n}(k)$ is a vector potential expressed as

$$
A_{n}(k)=i<u_{n}(k(t))\left|\nabla_{k}\right| u_{n}(k(t))>,
$$

which is known as the Berry Connection. Making a gauge transformation of the form

$$
\left|u_{n}(k(t))>\rightarrow e^{i \chi(k)}\right| u_{n}(k(t))>
$$

where $\chi(k)$ represents an arbitrary phase, gives the gauge dependent Berry Connection

$$
A_{n}(k) \rightarrow A_{n}(k)-\nabla_{k} \chi(k) .
$$


For an adiabatically slow process, the change in geometric phase $\gamma_{n}(t)$ is given by

$$
\chi(k(t=\tau))-\chi(k(t=0))
$$

between initial and final points. Assuming a cyclic evolution between two points along a closed path $C($ i.e., $k(0)=k(\tau))$,

$$
\chi(k(\tau))-\chi(k(0))=2 m \pi,
$$

where $m$ is an integer. So for a closed path $C$, the geometric phase is independent of the gauge and called as 'Berry Phase' which is

$$
\gamma_{n}=\oint_{C} d k \cdot A_{n}(k) .
$$

The Berry phase plays an important role in transport properties of Dirac surface states and will be further discussed later in relation to the the weak antilocalization in topological insulators.

\subsubsection{The Dirac Equation}

In 1928, Paul Dirac developed a relativistic equation for a spin $1 / 2$ particle ;

$$
H=c \mathbf{p} . \alpha+m c^{2} \beta
$$


where $m$ is the rest mass of the particle, $\mathbf{p}$ is the momentum, $\alpha$ and $\beta$ are the Dirac matrices satisfying

$$
\begin{gathered}
\alpha_{i}^{2}=\beta^{2}=1 \\
\alpha_{i} \alpha_{j}=-\alpha_{j} \alpha_{i} \\
\alpha_{i} \beta=-\beta \alpha_{i}
\end{gathered}
$$

The other pair of important matrices are known as Pauli matrices $\sigma_{i}(i=x, y, z)$ which also obey the anticommutation relation

$$
\sigma_{i} \sigma_{j}=2 \delta_{i j}
$$

with

$$
\sigma_{x}=\left(\begin{array}{ll}
0 & 1 \\
1 & 0
\end{array}\right), \sigma_{y}=\left(\begin{array}{cc}
0 & -i \\
i & 0
\end{array}\right), \sigma_{z}=\left(\begin{array}{cc}
1 & 0 \\
0 & -1
\end{array}\right) .
$$

In 1D, Dirac matrices $\alpha_{x}$ and $\beta$ are any two of Pauli matrices and in 2D they are equal to each other. In $1 \mathrm{D}$ we have

$$
\alpha_{x}=\sigma_{x}, \beta=\sigma_{z}
$$

and similarly in $2 \mathrm{D}$,

$$
\alpha_{x}=\sigma_{x}, \alpha_{y}=\sigma_{y}, \beta=\sigma_{z}
$$


Similar analysis for $3 \mathrm{D}$ results in $4 \times 4$ Dirac matrices which can also be written in terms of the Pauli matrices

$$
\alpha_{i}=\left(\begin{array}{cc}
0 & \sigma_{i} \\
\sigma_{i} & 0
\end{array}\right), \beta=\left(\begin{array}{cc}
\sigma_{0} & 0 \\
0 & -\sigma_{0}
\end{array}\right) \text {, }
$$

where $\sigma_{0}$ are $2 \times 2$ identity matrices. It becomes straightforward to solve the Hamiltonian in Eq. (1.11) by using these matrices. The corresponding energy eigenvalues are;

$$
E^{2}=m^{2} c^{4}+p^{2} c^{2},
$$

and in 3D there are two solutions for positive $\left(E_{+}\right)$and negative $\left(E_{-}\right)$energies with

$$
E_{ \pm}= \pm \sqrt{m^{2} c^{4}+p^{2} c^{2}}
$$

Negative solutions of Dirac equation suggested the existence of an antiparticle with a negative mass. In 1932, Carl D. Anderson detected the positron experimentally and he was awarded with the Nobel prize soon after. Despite the remarkable discovery of antimatter, the Dirac equation is inadequate for defining the topological matter as it is invariant under a transformation of mass $m \rightarrow-m$ by replacing $\beta \rightarrow-\beta$. A quadratic term is added to the modified Dirac equation, which makes a correction to the shape of Dirac cone. This modified Dirac equation can be written as,

$$
H=\nu p . \alpha+\left(m \nu^{2}-B \mathbf{p}^{2}\right) \beta,
$$

where $-B \mathbf{p}^{2}=-B\left(p_{x}^{2}+p_{y}^{2}+p_{z}^{2}\right)$ is the quadratic correction to the band gap $\mathrm{m} \nu^{2}$. The quantities $\nu, \mathrm{m}$ and $B$ have dimensions of speed, mass and reciprocal mass, respectively. The sign of mass $m$ or $m \nu^{2} \beta$ defines the spin distribution of the ground 

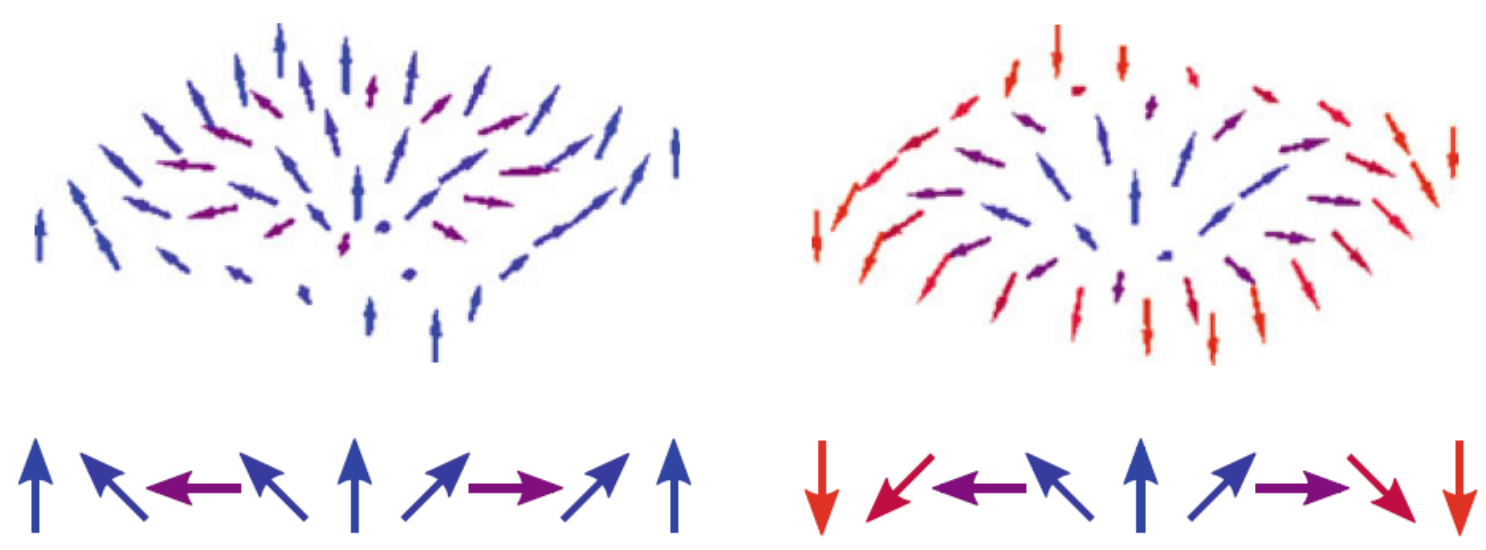

Figure 1.5: Left: Topologically trivial system with spins parallel to each other at $p=0$ and $p=\infty$. Right: Topologically nontrivial system with anti-parallel spin orientations at $p=0$ and $p=\infty$. Spin orientations along $p_{x}$ axis shown at the bottom left and right for the corresponding cases. Taken from reference [22].

state at $p=0$, whereas for large $p,-B \mathbf{p}^{2} \beta$ or the sign of $B$ matters. If $m B<0$, the increase in $p$ results in spin rotation from $z$ - direction to the direction of $\mathbf{p}$, then again back to the original $z$-direction. In this case, spins are parallel to each other and a topologically trivial configuration is formed (Fig. 1.5(left). On the other hand, if $m B>0$ and if $p$ increases along the $\mathrm{x}$-direction, then the spin rotates from $z$ - to $x$-direction of $\mathbf{p}$ at $p_{c}^{2}=m \nu^{2} / B$ and then to opposite z-direction at large $p$. This creates a topologically non-trivial state where spins at $p=0$ and $p=\infty$ are anti-parallel to each other as shown in Fig. 1.5(right). Therefore, the orientation of spin at $p=0$ and $p=\infty$ can be different for the cases of $m B>0$, and $m B<0$ and defines two topologically distinct cases. For the purpose of topological matter, $m B>0$ is the important case to be analyzed. The solution of Dirac equation for the case $m B>0$ gives helical edge states near the edge for a two dimensional TI and gives surfaces states for a 3D TI. 


\subsubsection{Dirac Surface States}

The solution of surface states for a system with $p_{x}=0$ and $p_{y}=0$ can be derived from the time-independent Schrodinger equation

$$
H(z)|\Psi\rangle=E|\Psi\rangle
$$

with [22]

$$
H(z)=C+D_{\perp} \hbar^{2} \partial_{z}{ }^{2}-i \nu_{\perp} \hbar^{2} \partial_{z} \alpha_{z}+\left(M+B_{\perp} \hbar^{2} \partial_{z}{ }^{2}\right) \beta .
$$

where the term $C+D_{\perp} \hbar^{2} \partial_{z}{ }^{2}=\epsilon(p)$, breaks the particle-hole symmetry between the conduction band and valence band. In the case of ${D_{\perp}}^{2}>B_{\perp}{ }^{2}$, the band gap closes and the system is metallic, whereas $D_{\perp}{ }^{2}<B_{\perp}{ }^{2}$ represents the insulating state. Therefore, it is important to have an effective Hamiltonian for the case $D_{\perp}{ }^{2}<B_{\perp}{ }^{2}$ in order to characterize Dirac surface states. We pick exponential trial wave functions as;

$$
\left|\Psi_{1}\right\rangle=\left(\begin{array}{c}
a_{1} \\
0 \\
b_{1} \\
0
\end{array}\right) e^{\lambda z}, \quad\left|\Psi_{2}\right\rangle=\left(\begin{array}{c}
0 \\
a_{2} \\
0 \\
b_{2}
\end{array}\right) e^{\lambda z}
$$

Matching boundary conditions yields

$$
\left(\begin{array}{cc}
M+B_{+} \lambda^{2} & -i \nu_{\perp} \lambda \\
-i \nu_{\perp} \lambda & -M+B_{-} \lambda^{2}
\end{array}\right)\left(\begin{array}{l}
a_{1} \\
b_{1}
\end{array}\right)=E\left(\begin{array}{l}
a_{1} \\
b_{1}
\end{array}\right)
$$

and

$$
\left(\begin{array}{cc}
M+B_{+} \lambda^{2} & i \nu_{\perp} \lambda \\
i \nu_{\perp} \lambda & -M+B_{-} \lambda^{2}
\end{array}\right)\left(\begin{array}{l}
a_{2} \\
b_{2}
\end{array}\right)=E\left(\begin{array}{l}
a_{2} \\
b_{2},
\end{array}\right)
$$


where $B_{ \pm}=B_{\perp} \pm D_{\perp}$. For $a_{1}$ and $b_{1}$, the secular determinant equation yields $\lambda= \pm \lambda_{1}$ and $\pm \lambda_{2}$. Since the wave functions vanish at $z \rightarrow 0$ and $z \rightarrow-\infty$ for topological insulators, we get

$$
\left|\Psi_{1}\right\rangle=\left(\begin{array}{c}
a_{1} \\
0 \\
b_{1} \\
0
\end{array}\right)\left(e^{\lambda_{1} z}-e^{\lambda_{2} z}\right)
$$

where $\lambda_{1}$ and $\lambda_{2}$ are given by

$$
\begin{aligned}
& \lambda_{1}=\frac{1}{2} \sqrt{\frac{\nu_{\perp}^{2}}{B_{+} B_{-}}}+\sqrt{\frac{1}{4} \frac{\nu_{\perp}^{2}}{B_{+} B_{-}}-\frac{M}{B_{\perp}}}, \\
& \lambda_{2}=\frac{1}{2} \sqrt{\frac{\nu_{\perp}^{2}}{B_{+} B_{-}}}-\sqrt{\frac{1}{4} \frac{\nu_{\perp}^{2}}{B_{+} B_{-}}-\frac{M}{B_{\perp}}},
\end{aligned}
$$

and similarly by setting $a_{2}=-a_{1}$ and $b_{2}=b_{1}$

$$
\left|\Psi_{2}\right\rangle=\left(\begin{array}{c}
0 \\
-a_{1} \\
0 \\
b_{1}
\end{array}\right)\left(e^{\lambda_{1} z}-e^{\lambda_{2} z}\right)
$$

with the eigenvalues $E=\frac{-D_{\perp} M}{B_{\perp}}$. Now we can construct the effective Hamiltonian for a system in general case $p_{x}=p_{y} \neq 0$ using the $p_{x}=p_{y}=0$ basis,

$$
H_{e f f}=\left(\begin{array}{cc}
\left\langle\psi_{1}|| H|| \psi_{1}\right\rangle & \left\langle\psi_{1}|| H|| \psi_{2}\right\rangle \\
\left\langle\psi_{2}|| H|| \psi_{1}\right\rangle & \left\langle\psi_{2}|| H|| \psi_{2}\right\rangle
\end{array}\right)=\epsilon_{0}(p)+\nu_{e f f}(p \otimes \sigma)_{z}
$$


The spin and momentum are locked in this effective model, which is the most important feature of Dirac surface states. To find the eigenstates, polar coordinates are introduced with $p=p_{x}^{2}+p_{y}^{2}$ and $\phi_{p}=\tan ^{-1}\left(p_{y} / p_{x}\right)$, so that $E_{ \pm}(p)=\epsilon_{0}(k) \pm \nu_{e f f} p$ yields

$$
\left|\Psi_{ \pm}\right\rangle=\frac{1}{\sqrt{2}}\left(\begin{array}{c} 
\pm e^{-i \phi_{p}} \\
i
\end{array}\right)
$$

The Berry phase around a closed loop will be

$$
\gamma_{ \pm}=\oint_{C} d \phi_{p}\left\langle\psi_{ \pm}\left|i \frac{\partial}{\partial \phi_{p}}\right| \psi_{ \pm}\right\rangle=\pi
$$

The accumulation of $\pi$ Berry phase is the key for understanding the weak antilocalization (WAL) behavior of surface states in a topological insulator.

\subsubsection{Weak Antilocalization (WAL)}

Weak antilocalization (WAL) occurs in materials that have intrinsic spin-orbit coupling. In TIs, large spin orbit coupling in the bulk results in spin-momentum locking of surface states. The transport experiments demonstrate that the TIs are in the diffusive regime where the mean free path is short and the mobility is low. In the diffusive regime, the electrons are scattered by defects and other electrons which changes their paths as they continue their motion. As a result of spin-momentum locking, whenever an electron scatters of an impurity its momentum changes and the spin rotates accordingly. As the electron closes a loop after scattering, the spin will be rotated by and angle of $\pi$. Similarly an electron scattering in the opposite rotational direction will have its spin rotated by $-\pi$. The phase difference between these two paths is $2 \pi$ which corresponds to an accumulation of $\pi$ Berry phase. There- 

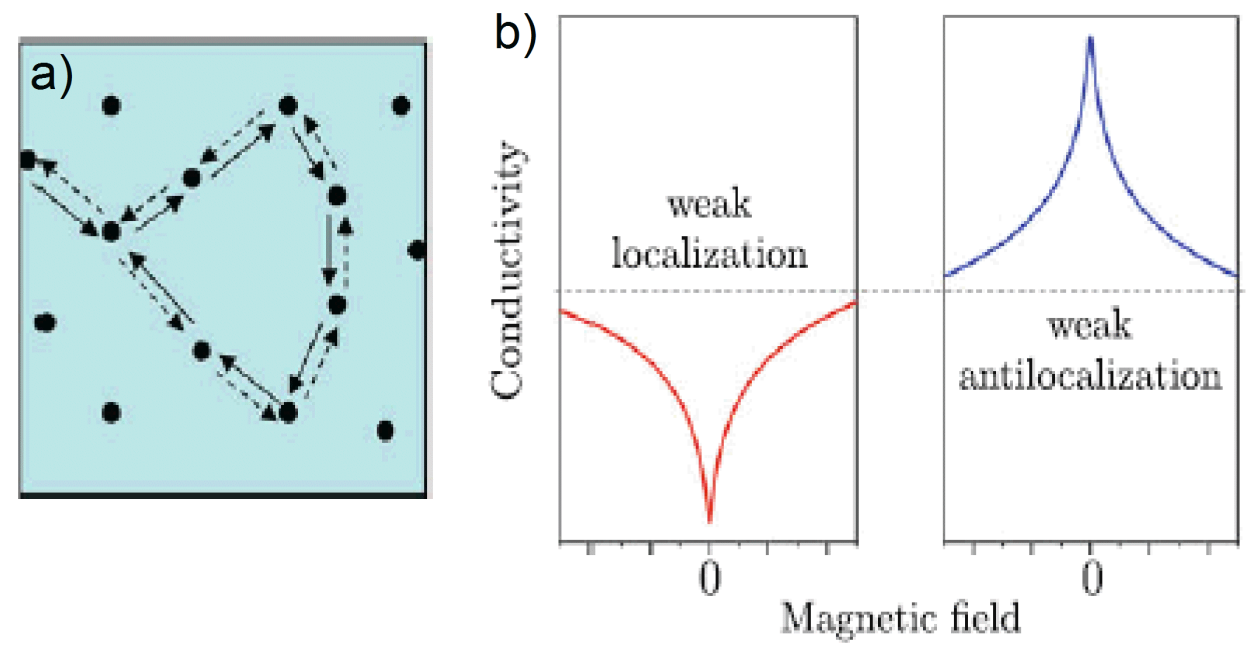

Figure 1.6: a) Illustration of the electron trajectory completing a clockwise or counterclockwise closed loop. Spin reorients itself with the direction of momentum at any point around the loop b) Left : Weak localization (WL) and positive magnetoconductance with the cusp, Right: Typical weak antilocalization (WAL) curve showing negative magnetoconductance with the cusp. Dashed line represents the Drude conductivity. From reference [22].

fore, the two paths interfere destructively and suppress the backscattering. The suppression of backscattering gives the conductivity an enhancement which can be destroyed by applying a magnetic field. In materials without spin orbit coupling, constructive interference enhances the backscattering and results in weak localization (WL), an opposite effect to WAL. The application of a magnetic field introduces a random Aharonov-Bohm phase shift into the system and disturbs the electronic phase coherence. In this case, there is no accumulation of $\pi$ Berry phase and all quantum localization effects are quenched. A negative magnetoconductance evolves as a function of field for WAL, and positive magnetoconductance for WL effects (Fig. 1.6). There are two important length scales related to the localization effects. Phase coherence length $L_{\phi}$, and spin-orbit scattering length $L_{S O}$. The distance that the electrons take to rearrange their spins after a scattering is called the spin-orbit 
scattering length $L_{S O}$ and it is equal to zero $\left(L_{S O}=0\right)$ in the infinite spin-orbit scattering limit as the spin immediately readjusts itself with the direction of momentum instantaneously. The phase coherence length $L_{\phi}$ is the distance that the electrons travel before they lose their coherence. The relative competition between these two scales determines the type of localization effect occurs in a material. For the surface states of TI, $L_{\phi} \gg L_{S O}$ and always results in WAL. Magnetic impurity doping can cause $L_{S O}>L_{\phi}$ and results in WL in some TI systems. In magnetically doped TIs WAL - WL crossover can be explained by the relative ratio of the gap opening to the Fermi energy. The Berry phase is given by [23]

$$
-i \int_{0}^{2 \pi} d \phi\left\langle\psi_{k}(r)\left|\frac{\partial}{\partial \phi}\right| \psi_{k}(r)\right\rangle=\pi\left(1-\frac{\Delta}{2 E_{F}}\right)
$$

which yields $\pi$ for WAL $\left(\Delta / 2 E_{F}=0\right)$, and $2 \pi$ for WL $\left(\Delta=2 E_{F}\right)$. The ratio $\Delta / 2 E_{F}$ can be tuned via doping or gating to investigate the transition in magnetically doped systems.

For a diffusive system with spin orbit interaction, 2D magnetoconductance was studied by Hikami, Larkin and Nagaoka (HLN). The quantum correction to the Drude conductivity is given by HLN equation [24],

$$
\Delta \sigma_{\perp}(B)=\alpha \frac{e^{2}}{2 \pi^{2} \hbar}\left[\psi\left(\frac{\hbar}{4 e L_{\phi}^{2} B}+\frac{1}{2}\right)-\ln \left(\frac{\hbar}{4 e L_{\phi}^{2} B}\right)\right],
$$

where $\alpha$ is a preconstant, $\psi$ is the digamma function and $L_{\phi}$ is the phase coherence length which is related to the dephasing field, with $B_{\phi}=\hbar /\left(4 e L_{\phi}^{2}\right)$. There are three important cases in HLN theory:

i) If there is neither spin orbit interaction nor magnetic scattering occurs, then $\alpha=1$ corresponding to the orthogonal case which results in weak localization (WL) 
ii) If magnetic scattering is strong, $\alpha=0$, which is called the unitary case.

iii) If there is no magnetic scattering and the spin orbit interaction is strong, $\alpha=-0.5$, which is referred to as the symplectic case.

The surface of a topological insulator can be treated with HLN theory as a two dimensional random scattering potential. Strong spin orbit coupling always results in dominant WAL with a prefactor $\alpha \approx-0.5$. Since the spin orbit scattering is sufficiently strong, $\Delta R=-R^{2} \Delta \sigma$ is positive and always results in positive magnetoresistance (negative magnetoconductance).

\subsubsection{Electron-Electron Interaction (EEI) in Disordered Sys- tems}

The effect of electron electron collisions in disordered systems has been extensively studied to understand the temperature and field dependence of conductivity $[25,26]$. The low temperature dephasing time $\left(\tau_{\phi}\right)$ in the quantum interference regime has been studied to analyze the different scattering mechanisms in disordered conductors [27]. The short dephasing time observed in disordered conductors is a major obstacle to achieve high speed operation of solid state devices. Typically, low field magnetoconductance is used to extract the dephasing times (or coherence lengths). There are two important sources of dephasing in a disordered conductor: i) electron-phonon interactions which dominate at high temperatures temperatures, and ii) electron-electron interactions which dominate at low temperatures ( typically 1-10 $\mathrm{K}$ in 2D disordered metals) The theory developed by Altshuler and Aronov predicts that the dephasing at low temperatures in dirty metals is dominated by electron-electron scattering. An electron's interaction with the fluctuating electromagnetic fields from nearby electrons causes an enhanced scattering and increases 
the resistance [26]. A sudden change in the charge distribution in a disordered metal can not be screened immediately. Since the electrons are in diffusive regime, they need time to screen the charge distribution. A slow diffusion process leads to a reduced screening. As a result, an effective electron electron interaction (EEI) builds up due to the exchange interaction between electron pairs [27]. This introduces a correction to the resistance of the system at low temperatures. Even though weak localization has a similar effect on the resistance, the physical interpretation is different. It is important to note that the Altshuler-Aronov mechanism leads to a result that is mathematically identical to the HLN formalism. The reason is that both mechanisms rely on interference of states due to scattering in two-dimensions. For a disordered conductor, the dephasing rate (2D) [25],

$$
\tau_{\phi}^{-1}=k_{B} T \frac{e^{2} R_{\square}}{2 \pi \hbar^{2}} \ln \left(\frac{\pi \hbar}{e^{2} R_{\square}}\right)^{2 / 3}
$$

where $R_{\square}$ is the sheet resistance. The $2 \mathrm{D}$ theory predicts a dephasing rate of $\tau_{\phi}{ }^{-1} \approx$ $T$ (or $L_{\phi} \approx T^{-0.5}$ since $\mathrm{L}_{\phi}={\sqrt{D \tau_{\phi}}}_{\text {) }}$ in good agreement with the experimental observations of the transport measurements of TI thin films. In zero field, the EEI and interference corrections to the conductivity $\sigma(T, H=0)$ are additive to the lowest order and can be written as [28],

$$
\Delta \sigma=-\frac{\Delta R}{R} \frac{1}{R_{\square}}=\frac{e^{2}}{2 \pi^{2} \hbar}\left[\alpha p+\left(1-\frac{3}{4} \widetilde{F}\right)\right] \ln \left[\frac{T}{T_{0}}\right]
$$

where $T_{0}$ is a temperature parameter, $p$ is the temperature index of Thouless length (ie: thermal length) $L_{T h}$, with $p$ depending on the collision mechanism. The preconstant $\alpha=1$ for an orbitally non degenerate free electron gas. The second term $\frac{3}{4} \widetilde{F}$ is the Coulomb interaction (or EEI) term with $\widetilde{F}$ related to the Hartree screening 
length. The experiments with TIs demonstrated that the EEI correction is also logarithmic with decreasing temperature. The application of perpendicular magnetic field quenches the quantum corrections and leaves only the electron-electron interaction term. Therefore, it is important to analyze the conductivity in the zero and non-zero fields to separate the two contributions for the case of TI thin films. 


\section{Chapter 2}

\section{Growth and Characterization of $\mathrm{Bi}_{2} \mathrm{Se}_{3}$ and $\mathrm{Bi}_{2-x} \mathrm{Mn}_{x} \mathrm{Se}_{3}$ Thin}

\section{Films}

\subsection{Thin Film Growth and Fabrication}

\subsubsection{Molecular Beam Epitaxy (MBE)}

Molecular beam epitaxy is a technique for growing thin films of semiconductors [29], metals [30], and insulators [31]. Very low background pressures $\left(\mathrm{P} \approx 10^{-10}\right.$ Torr) allow a contaminant-free environment for the growth of high quality thin films. Materials of interest are stored in non-reactive crucibles that reside in Knudsen cells (k-cells) which operate under thermal processes. The crucibles are surrounded by resistive filaments which heat and evaporate the material. Temperature of a k-cell is read via a thermocouple with a precision approximately $\pm 0.1^{\circ} \mathrm{C}$. The molecular beam of elements evaporates from k-cells and arrives at surface of a substrate and 
crystallizes. The growth rate is low enough (typically 0.1 monolayer/s) to allow surface migration of species at the substrate surface. As a result, atomically smooth and sharp surfaces can be achieved.

The mean free path of a molecule is defined as the average distance traveled between the successive collisions. In a high vacuum system the mean free path of molecules is given by [32]:

$$
L_{b}^{-1}=\sqrt{2} \pi n_{b} d_{b}^{2}+\pi n_{g} d_{b g}^{2} \sqrt{1+v_{g}^{2} / v_{b}^{2}},
$$

with $d_{b g}=\left(d_{b}+d_{g}\right) / 2$ and where $n_{b}, d_{b}$, and $v_{b}$ are concentration, diameter and average velocity of the molecules in the molecular beam and $n_{g}, d_{g}$, and $v_{g}$ are concentration, diameter and average velocity of the molecules of the residual gas in the reaction chamber. In a typical MBE chamber, the mean free path is on the order of a thousand meters, and much larger than the diameter of the chamber itself. Therefore, molecules do not collide and interact until they hit the substrate. Moreover, the ultra-high vacuum conditions allows the utilization of various in-situ techniques, including RHEED [33], Auger electron spectroscopy (AES) [34], spectroscopic ellipsometry [35], and laser interferometry [36] among others.

What distinguishes MBE from other deposition techniques is the precise control over the beam fluxes and growth conditions. Mechanical shutters in front of beam sources are used to control incoming molecular beams and make it possible to arrange different ways of growing a multilayered structure, or ensures a precise doping in a material. In the growth of undoped $\mathrm{Bi}_{2} \mathrm{Se}_{3}$ and $\mathrm{Bi}_{2-x} \mathrm{Mn}_{x} \mathrm{Se}_{3}$ thin films, we used a co-deposition method and opened all shutters simultaneously. 


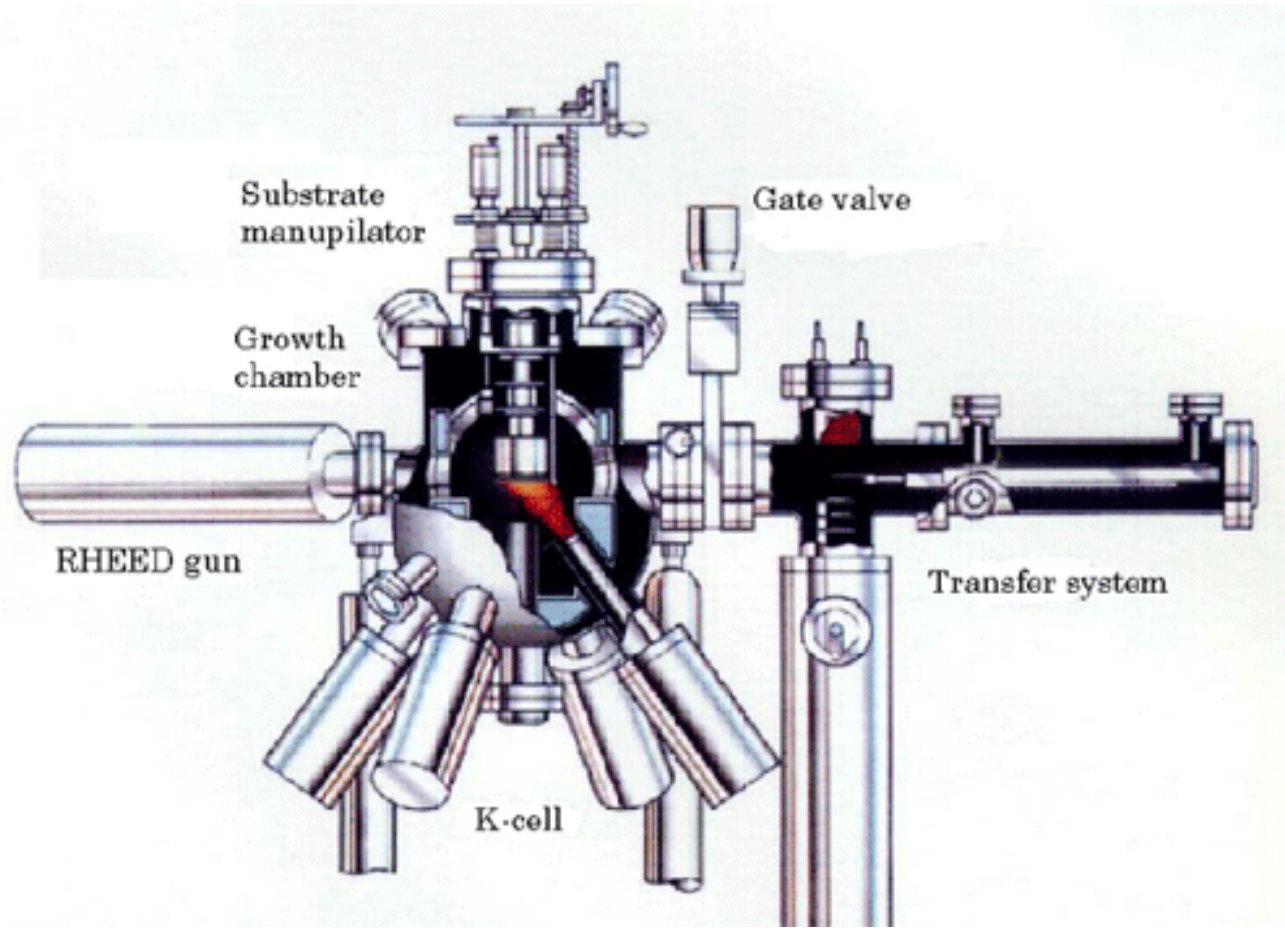

Figure 2.1: Picture of a typical MBE chamber. RHEED apparatus, k-cells for source materials, manipulator, a gate valve to isolate UHV chamber from the transfer entry lock can be seen in the figure. Pump and quartz crystal microbalance are not displayed [37].

\subsubsection{Photolithography}

Photolithography is a technique used to remove parts of the film which are not necessary for the measurement. To start with, a clean surface is assured by sonicating the film typically around 30 seconds in acetone. Then an appropriate photoresist is applied on the sample surface with spin coating method. The film is spun at high speed to distribute the photoresist evenly on the surface. After spin coating, the sample is baked on a hot plate at $95{ }^{\circ} \mathrm{C}$ for four minutes. The baking process assures the hardening of photoresist and improves adhesion to the film. There are two types of photoresists that can be used as shown in Fig. 2.2, positive and negative one. It is necessary to use the positive photoresist if an exact copy of the pattern is desired on 


\begin{tabular}{|cc|}
\hline Photolithography step & Time $(\mathrm{s})$ \\
\hline Ultrasonicator & 30 \\
Spin coater & $60(\mathrm{~s}=2160 \mathrm{rpm})$ \\
Soft bake & $240\left(\mathrm{~T}=95{ }^{\circ} \mathrm{C}\right)$ \\
UV exposure & 111 \\
Developer & 60 \\
\hline
\end{tabular}

Table 2.1: Details of photolithography process used in patterning $\mathrm{Bi}_{2} \mathrm{Se}_{3}$ and $\mathrm{Bi}_{2-x} \mathrm{Mn}_{x} \mathrm{Se}_{3}$ thin films.

sample surface. In these resists, exposure to UV light changes the chemical structure of the photoresist polymer and it becomes more soluble in developer. The negative resists work in the opposite way: they stay on the parts where they are exposed to UV light and the developer removes only unexposed portions. After finishing spin coating and baking process with a positive photoresist, the sample is mounted on the photolithography equipment with a specially designed mask. After aligning the sample with the desired pattern, the automatic exposure unit is set for a certain time. Pressing start button causes the shutter to open and sample is exposed to UV light for specific amount of time. After exposure, the sample is rinsed in developer for 60 seconds. This washes away all photoresist that was exposed to UV light and leaves the rest. Then the sample is rinsed in DI water for a few seconds. After this process, a pattern is clearly seen on the sample surface. We patterned our films with a specially designed mask, and used a positive photoresist (AZ 5214). All films were exposed to UV light for 111 seconds. The parameters related to the patterning process are summarized in Table 2.1. 


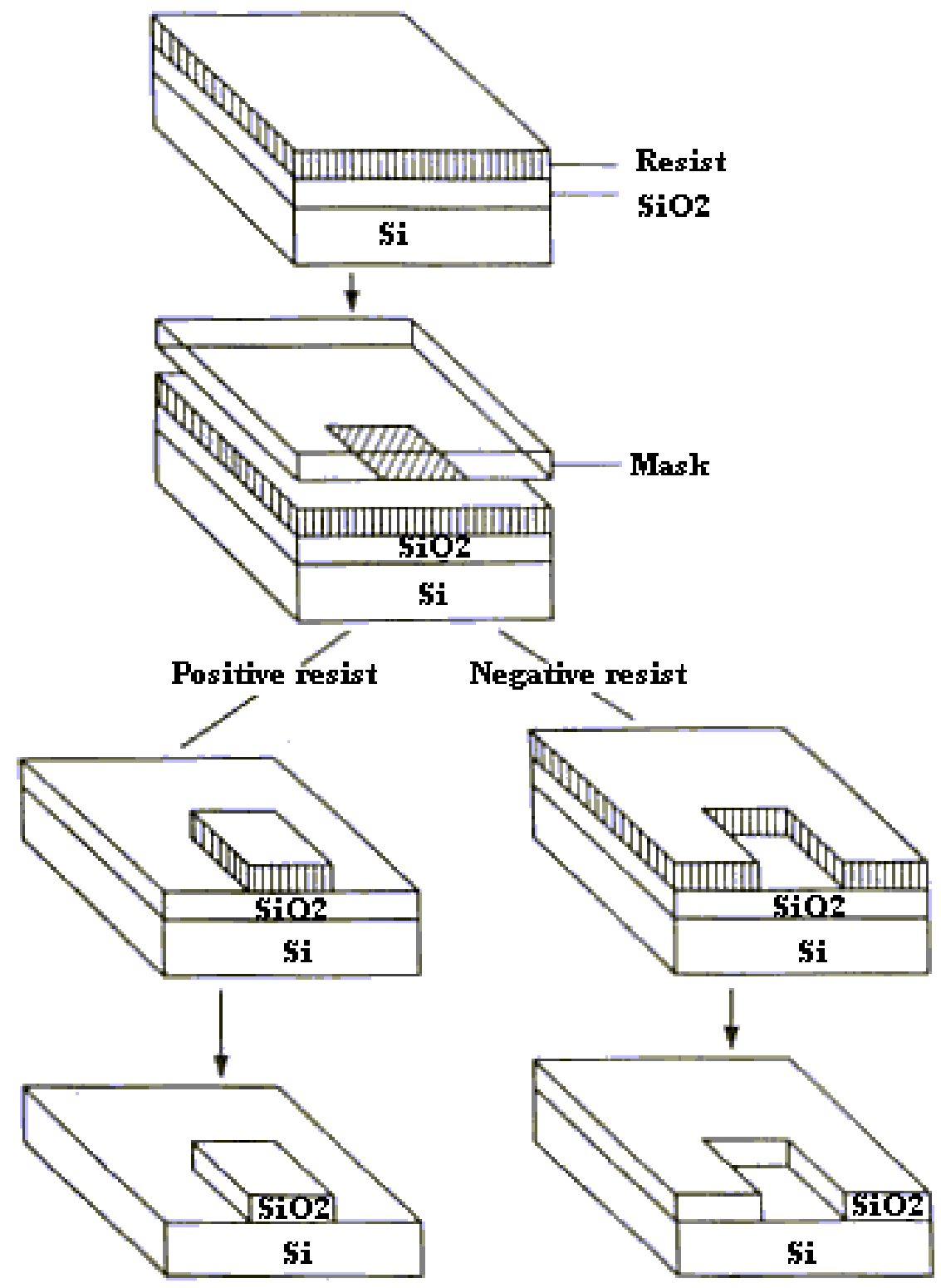

Figure 2.2: Patterning with positive and negative photoresists. From reference [38]. 


\subsubsection{Argon Ion Milling}

Following developing process in photolithography, it becomes important to etch away the unwanted parts of the film. The etching process must be considered very carefully since a remaining portion of an unwanted film would result in electrical shorts through the contacts. There are two different etching processes. In wet etching, a specific acid or base solution is used to remove the extra material. The advantage of this technique is the time efficiency. The films can be etched in 1-3 seconds. However, it is very difficult to estimate the etching periods for different materials. Therefore, significant damage on the electrical contacts may occur. The second etching technique is known as dry etching. Dry etching involves bombardment of the sample surface with an ionized inert gas such as argon. Typically a series of grids at a specific potential is used to accelerate Ar ions. A neutralizer is used to neutralize Ar ions to reduce charging effects. The beam is directed on the sample surface and removes unwanted material atoms from the surface. We used Ar ion milling to etch away the conducting parts of our films after the developing step. All of the $\mathrm{Bi}_{2} \mathrm{Se}_{3}$ and $\mathrm{Bi}_{2-x} \mathrm{Mn}_{x} \mathrm{Se}_{3}$ thin films were fabricated, patterned, and etched in the same way.

\subsection{Thin Film Characterization Techniques}

\subsubsection{Reflection High Energy Electron Diffraction (RHEED)}

RHEED is an in-situ characterization technique used to study the surface quality of an MBE grown thin film. An electron beam with an energy in the $5-40 \mathrm{keV}$ range is aimed at the sample's surface with a very shallow angle of incidence (typically 1 ${ }^{\circ}$ ) which interacts with the atoms on the topmost layers of a thin film. The grazing 
angle of incidence results in a very small diffraction contribution from the planes that are perpendicular to the film surface, whereas the diffraction from the planes parallel to the film surface can be detected with high precision [39]. The wavelength of the electrons in the incident beam is given by the de Broglie wavelength, which can be expressed as [32]

$$
\lambda \approx \frac{12.247}{\sqrt{V\left(1+10^{-6} V\right)}} \AA
$$

where $V$ is the accelerating voltage of electron beam in volts. The typical electron wavelengths lie between 0.06 and $0.2 \AA$.

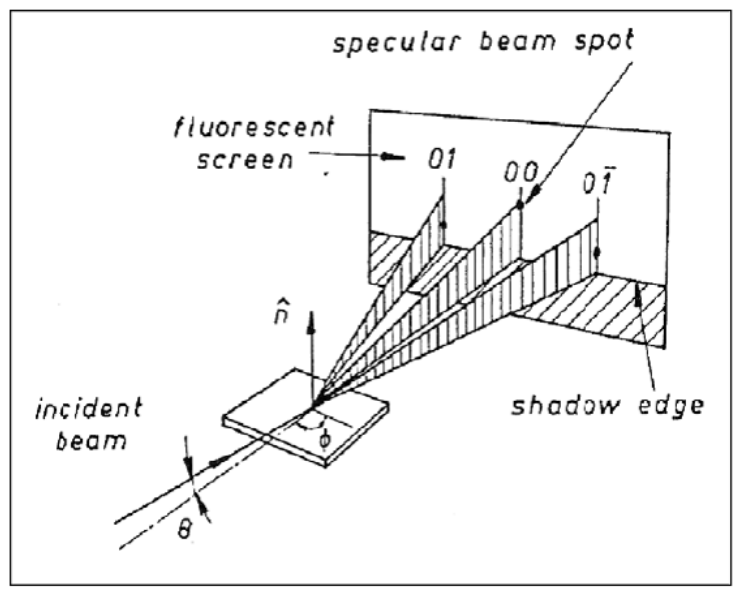

Figure 2.3: Typical RHEED schematic where phosphorous screen is located at $180^{\circ}$ relative to the electron beam gun. From reference [32].

The term " layer-by-layer" growth (Frank-van der Merwe) is a special type of growth mode in which atoms start to form next atomic monolayer after completion of the previous one. During this process, the specular beam of the diffraction pattern oscillates in time, a phenomenon widely known as RHEED oscillations. III-V group of compound semiconductors are known to grown in epitaxial fashion on appropriate substrates. A well known example is the growth of GaAs, for which RHEED 
oscillations are shown in Fig. 2.4. Each oscillation corresponds to the growth of a monolayer of GaAs.

There are two ways to achieve layer-by-layer growth and obtain RHEED oscillations [40]. The first method involves operating the source shutters in succession, so as to deposit different layers in the material sequentially. Another method involves opening the source shutters simultaneously. The growth $\mathrm{Bi}_{2} \mathrm{Se}_{3}$ occurs in a special way called quintuple layer epitaxy [41] which consists of Se-Bi-Se-Bi-Se atomic layers successively. Therefore, at formation of each quintuple layer there is one corresponding RHEED oscillation with the period equals to the time necessary to complete a full quintuple layer. I used the second method, and achieved quintuple layer by quintuple layer growth (ql-by-ql) of pure $\mathrm{Bi}_{2} \mathrm{Se}_{3}$ thin films by co-depositing bismuth and selenium.

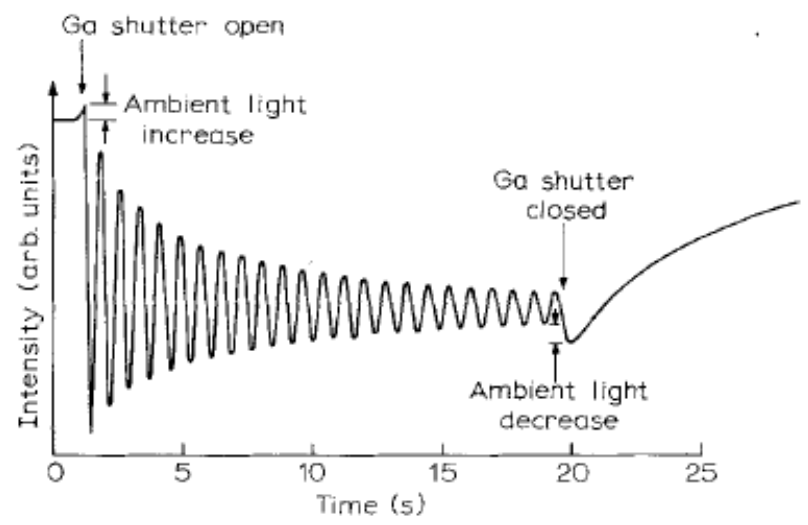

Figure 2.4: RHEED intensity of oscillations for GaAs thin film. The period of oscillations exactly corresponds to the growth of a single monolayer (i.e. a complete layer of $\mathrm{Ga}+\mathrm{As}$ atoms.). From reference [40]. 


\subsubsection{X-ray Diffraction (XRD)}

$\mathrm{X}$-ray diffraction is one of the most common techniques used to study the crystal structure of a material. It was first discovered by Max von Laue in 1912, and it is now widely used in nearly all areas of material science research. X-ray diffraction is a direct tool to study materials structure due to the fact that wavelength of x-rays are on the order of one Angstrom and comparable to the lattice spacing of most materials.

Crystallinity in a material guarantees the formation of stacking layers in which atoms arrange themselves in a specific ordering. Therefore, the atomic ordering enables the reflection and interference between scattered x-rays from the atomic planes. [42] When a monochromatic beam of x-rays is directed to a crystalline sample, it results in reflections and constructive interference of $\mathrm{x}$-rays from adjacent planes if as Bragg's condition is met, such that [43]:

$$
2 d_{h k l} \sin \theta=\lambda,
$$

where $d_{h k l}$ is the lattice spacing in reciprocal space, $\theta$ is the angle of reflection between the sample surfaces normal and outgoing x-rays, and $\lambda$ is the wavelength of $\mathrm{x}$-rays. Equivalently, one can write

$$
\frac{\mathbf{S}-\mathbf{S}_{0}}{\lambda}=\mathbf{H}_{h k l},
$$

where $\mathbf{S}$ and $\mathbf{S}_{0}$ are the unit vectors for reflected and incident beams and $\mathbf{H}_{h k l}$ is the reciprocal lattice vector perpendicular to $h k l$ plane. In order for diffraction to occur, the reciprocal lattice vector should coincide with the momentum transfer vector of the scattered x-rays. Since x-ray scattering from the lattice is mostly elastic, the initial and final momenta of the scattered photons have the same magnitude 


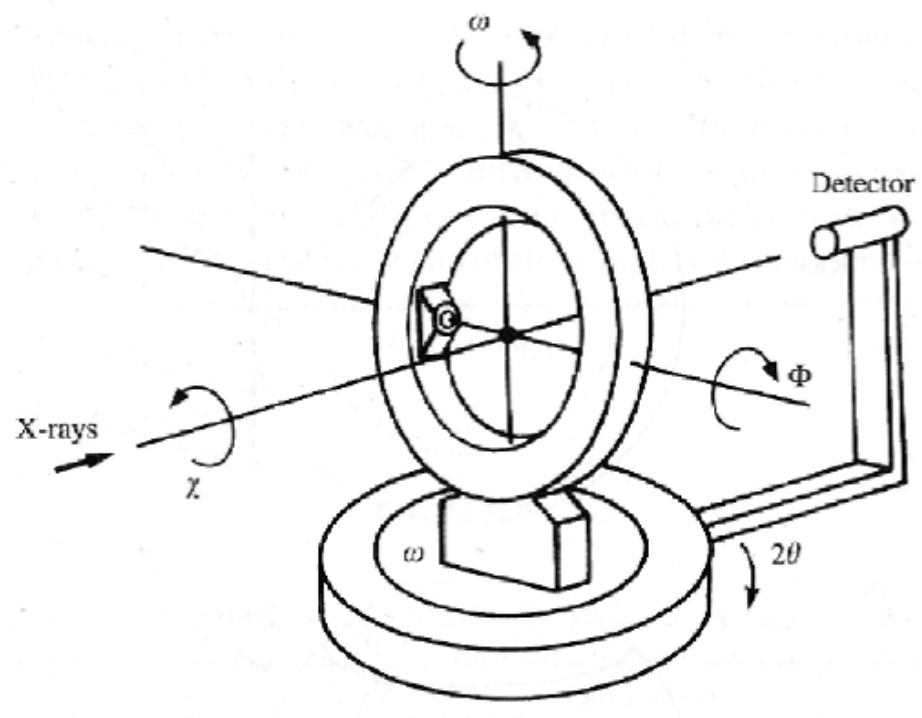

Figure 2.5: Four circle x-ray diffractometer. From reference [44].

but differ in direction. This implies that the reciprocal lattice vector must connect two points in reciprocal space that lie on a sphere of radius $k=2 \pi / \lambda$, such that $\mathbf{H}_{h k l}=\vec{k}_{f}-\vec{k}_{i}=\Delta \vec{k}$. (Figs. 2.6 and 2.7) To measure the out-of-plane x-ray diffraction from a crystal, a sample is mounted on a diffractometer as shown in Fig. 2.5. The angle $\chi$ is set to $\chi=90^{\circ}$ where the reference $\chi=0^{\circ}$ is defined at the position where the surface normal is vertical. However, in-plane XRD measurements require setting $\chi$ to a specific value in order to detect corresponding reflection from the crystal. The relations between diffraction angles, lattice structure and lattice constants can be derived for different crystal structures [42].

\subsubsection{X-ray Reflectivity (XRR)}

X-rays become very sensitive to the interlayer thickness and roughness when the angle of incidence is less than $10^{\circ}[45,46]$.

After reflecting off the surface of a sample, x-rays interfere with each other due 


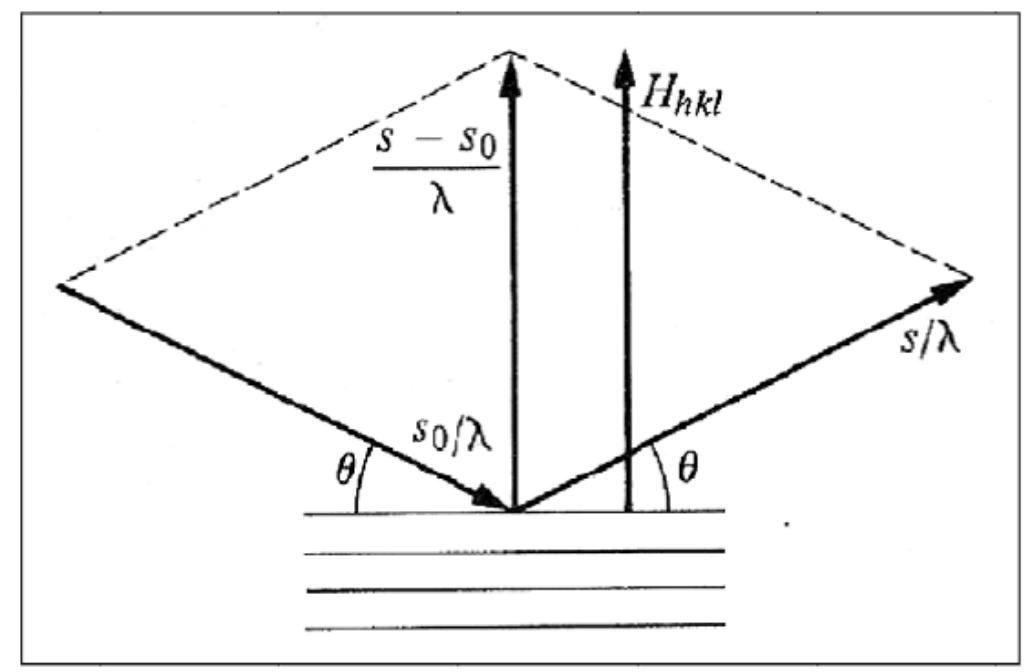

Figure 2.6: Schematic of x-ray diffraction. $S$ and $S_{0}$ are the incident and scattered x ray unit vectors. $H_{h k l}$ is reciprocal lattice vector perpendicular to $h k l$ plane. From reference [42].

to scattering from different interfaces. As a result, an oscillatory pattern is observed in the $\theta-2 \theta$ scan known as Kiessig fringes [47]. Parratt developed a recursive algorithm by using an optical approach based on the Fresnel equations [48]. In 1980, L. Nevot and P. Croce defined an exponential decay factor proportional to the square of the vertical roughness at each interface in order to take into account the interlayer roughness. [49]. In this work, I used Parratt's algorithm to estimate thickness and interface roughness parameters for all of our thin films.

\subsubsection{Atomic Force Microscopy (AFM)}

Atomic force microscopy is a widely used technique to study the surface topography of thin films. Usually a cantilever with a nanosize tip is held at a position above the sample surface. The tip is attached to a piezoelectric material which results in vertical and horizontal displacements when a voltage is applied. A laser beam is 


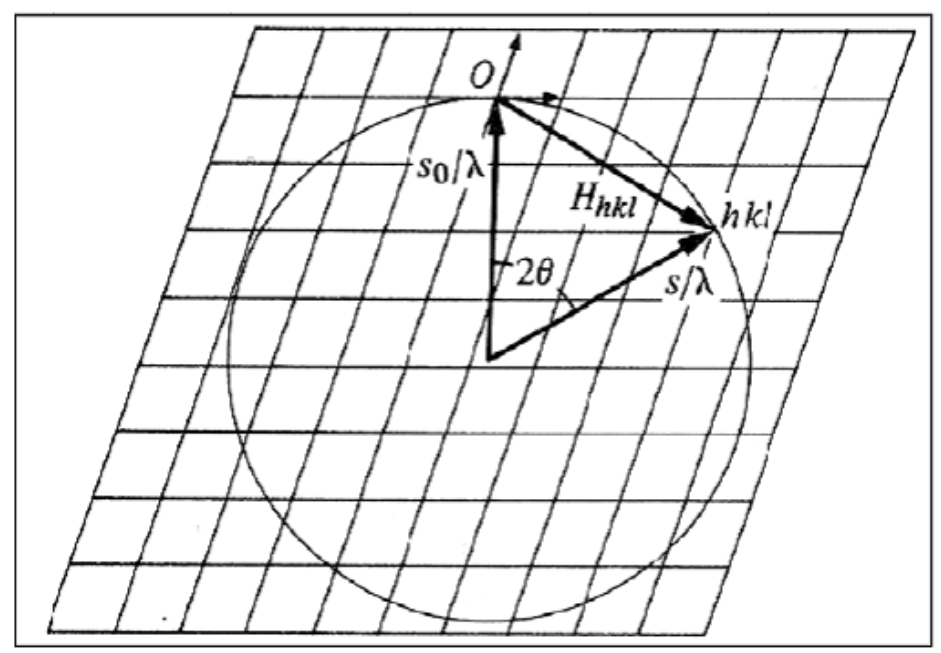

Figure 2.7: Diffraction condition is satisfied for any point hkl crossing the Ewald sphere. From reference [42].

reflected off from the back of the cantilever and this beam generates a voltage in a diode array detector. A displacement of the beam on the array is interpreted as a vertical displacement of the tip. Therefore, diode array can be used as a standard feedback mechanism to adjust the force or the distance between the cantilever and the surface.

In contact mode, the interaction between the tip and sample surface causes a deflection in the tip's position and this is adjusted by use of piezoelectric material. The amount of the vertical displacement adjusted recorded to map the topography of sample surface. This technique, also known as contact mode scanning, has several drawbacks especially for soft samples. The tip contaminates over time and produces artifacts and can damage the sample surface in contact.

In tapping mode, the cantilever is driven at its natural frequency, typically around $50-400 \mathrm{kHz}$. Due to this oscillating character, the tip comes in contact with sample surface only sporadically. The interaction between sample surface and the tip causes a change in oscillation amplitude. The topography of the sample surface can be 

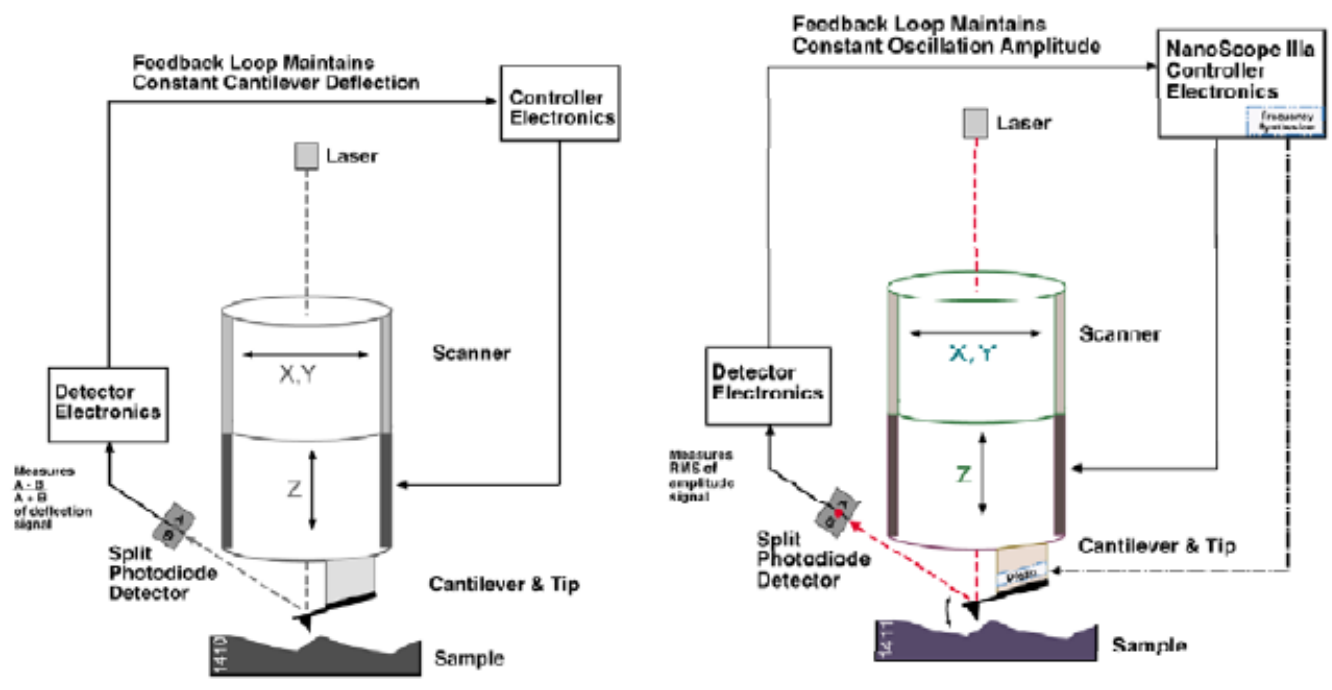

Figure 2.8: Schematics for two AFM modes: Contact mode (left) and tapping mode (right). From reference [50].

extracted by measuring the changes in oscillation amplitude using diode array. The two different modes of AFM setup are shown in Fig. 2.8.

\subsubsection{X-ray Photoelectron Spectroscopy (XPS)}

X-ray photoelectron spectroscopy (XPS) is used to estimate the stoichiometric composition of materials. An x-ray beam is directed to the sample and it knocks out some electrons from the material. Information about the atomic species in material can be directly learned by detecting the energy spectrum of these ejected electrons. Similar to other electron spectroscopy techniques, XPS is also a surface sensitive technique due to the short mean free paths of electrons in solids. The photo-emission process during XPS can be described by the photoelectric effect, so that

$$
E_{b}=h v-E_{k}-\phi
$$




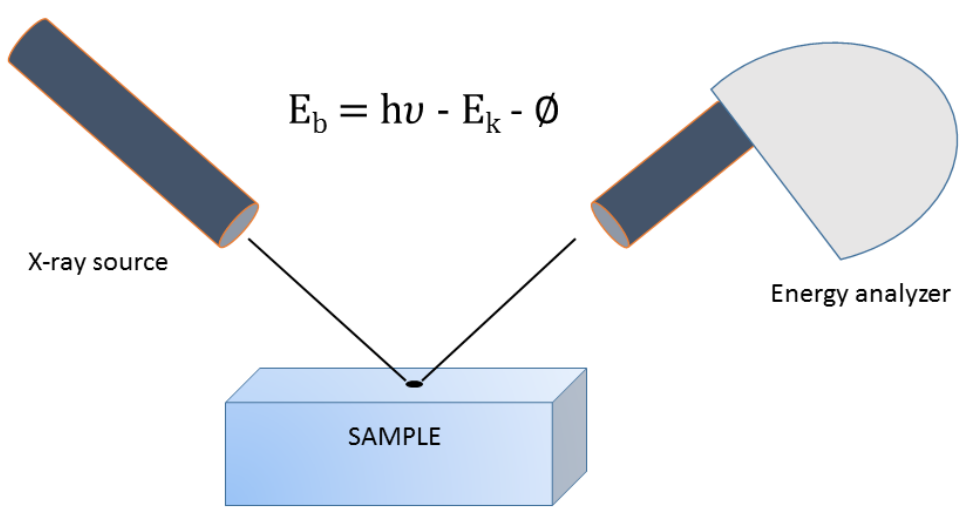

Figure 2.9: Illustration of a typical XPS setup.

where $E_{b}$ is the binding energy of electrons, $h v$ is the photon energy, $E_{k}$ is the kinetic energy of electrons measured by spectrometer, and $\phi$ is the spectrometer work function, respectively. The sensitivity of XPS to excite core shell electrons in a material allows accurate identification of chemical composition. The quantitative comparison of stoichiometry is achieved by comparing relative peak intensities of different core levels. Also, information about the chemical bonding states is obtained by measuring energy shifts of core level peaks. Finally, the areas under the peak intensities are integrated and scaled with a sensitivity factor to determine the coverage of a chemical species in the material. A typical XPS setup is shown in Fig. 2.9.

XPS analysis was used to determine the concentration of $\mathrm{Mn}$ in $\mathrm{Bi}_{2-x} \mathrm{Mn}_{x} \mathrm{Se}_{3}$ thin films. We acquired the XPS spectrum for corresponding Bi-4f, Se-3d, Mn-2p states and estimated the Mn concentration by comparing it to the Bi coverage in our films. 


\subsubsection{Extended X-ray Absorption Fine Structure (EXAFS)}

Extended x-ray absorption fine structure (EXAFS) is a widely used spectroscopy technique to probe the local structure of an atom in a material by means of analyzing the x-ray absorption spectrum. [51]. In an EXAFS experiment, the absorption of radiation is measured as a function of energy and it is defined as

$$
\mu(E)=\ln \left(\frac{I_{1}}{I_{2}}\right),
$$

where $\mu(E)$ is the absorption coefficient, $I_{1}$ and $I_{2}$ represents the intensities of incoming and transmitted waves. The main mechanism for the EXAFS is the photoelectric effect. When the energy of incoming x-rays match the energy difference between a core level (energy of 1s(K-shell), 2s(L-shell) or 2p(M-shell)) and the Fermi level, the absorption increases rapidly (Fig. 2.10). Incoming x-ray photon energy is absorbed and core electron is excited above the Fermi level with kinetic energy. Emitted photoelectrons propagate in the crystal lattice and interacts with the surrounding atoms. Forward propagating waves and the back-scattered waves interfere which results in an oscillatory pattern in the absorption coefficient $\mu(E)$ known as EXAFS spectra. These oscillations (Fig. 2.11) can be analyzed to obtain information about structural parameters such as bond lengths and Debye-Waller factors. 


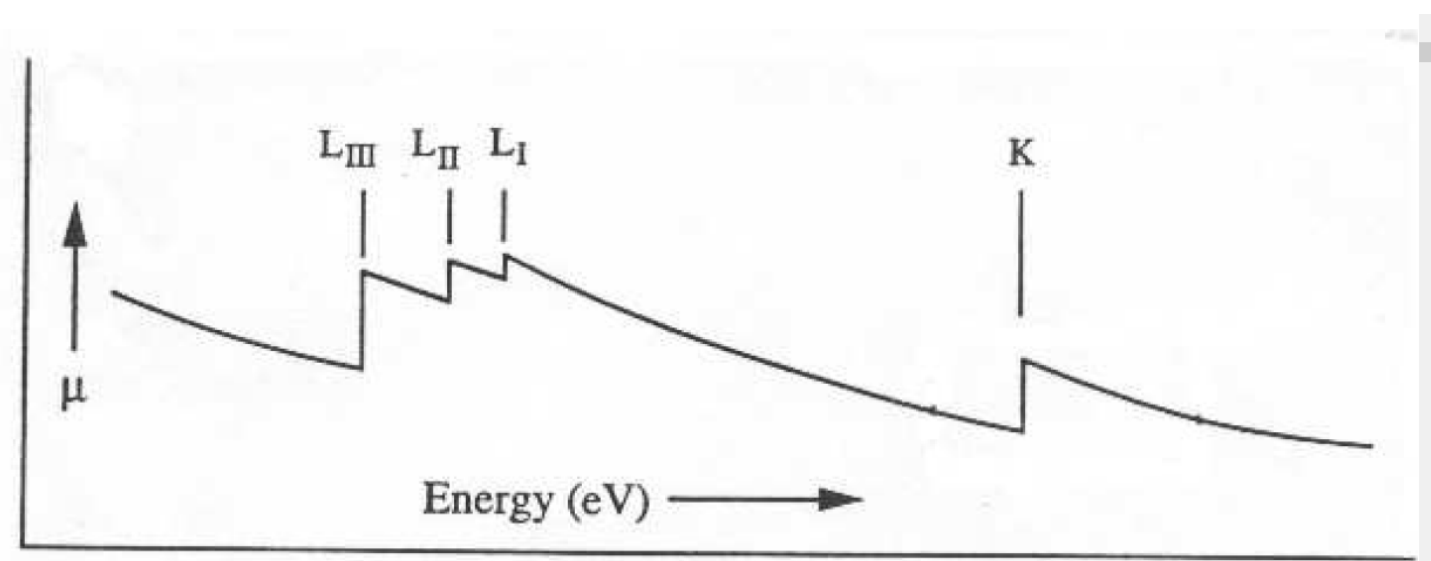

Figure 2.10: Energy dependence of x-ray absorption coefficient, Ref. [52].

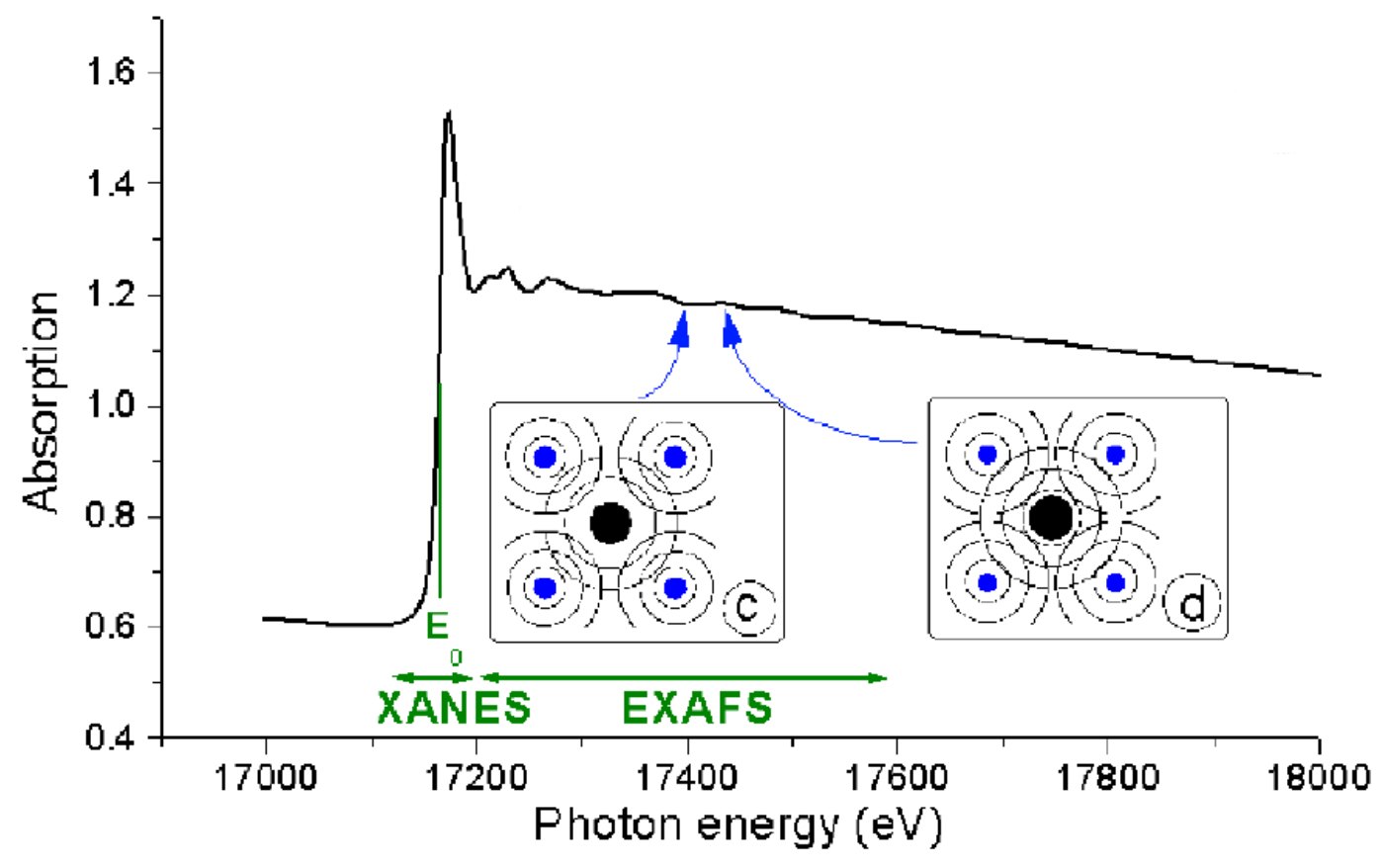

Figure 2.11: Interference in an EXAFS experiment, Ref. [52]. 
The EXAFS function is given by

$$
\chi(E)=\frac{\mu(E)-\mu_{i}(E)}{\Delta_{\mu_{i}}\left(E_{0}\right)},
$$

where $\mu(E)$ is absorption coefficient, $E_{0}$ is absorption edge energy, $\Delta_{\mu_{i}}$ absorption edge step and $\mu_{i}$ is an atomic background related to the absorption on free ion in the material, which excludes the oscillations due to interference. Also, it is convenient to define the wavevector $k$ of photoelectron and the EXAFS function $\chi(k)$. In order to treat the the oscillations at high $k$ values, the function $\chi(k)$ is weighted by a factor of $k^{p}$, where $p$ takes values of 1,2 or 3 . Indeed, $\chi(k)$ function includes all of the possible paths for the photoelectron propagation. When a photoelectron is emitted from an atom, it starts propagating and the wave associated with it is reflected from a neighboring atom. The electron can bounce back and scatter from the original atom or another atom nearby. So the photoelectron can scatter many times before it returns to the original atom. All the possible paths (trajectories) contribute to the EXAFS function $\chi(k)$, and the total $\chi(k)$ can be written as

$$
\chi(k)=S_{0}^{2} \mathcal{R} \sum_{\text {shell }} \frac{N_{\text {shell }} F_{\text {eff }}}{k R_{\text {shell }}^{2}} \exp (-2 r / \lambda) \sin \left(2 k R_{\text {shell }}+\phi_{\text {eff }}+\phi_{c}\right) \exp \left(-2 k^{2} \sigma^{2}\right)
$$

where $N_{\text {shell }}$ is the number of atoms in each shell, $S_{0}^{2}$ is amplitude, $R_{\text {shell }}$ is the distance between central and surrounding atom, $\sigma^{2}$ is Debye-Waller factor representing the disorder in neighboring distance, $\lambda$ is mean free path, $\rho$ is central atom loss factor, $F_{\text {eff }}$ is effective backscattering amplitude, $\phi_{e f f}$ is a phase correction, $\phi_{c}$ is the phase shift of central atom, and $\mathcal{R}$ is the the total loss factor for central atom. The data and fits of EXAFS spectrum can be expressed in terms of the Fourier transform of $\chi(k)$, which is $\chi(R)$. The peak and oscillations in $|\chi(R)|$ are used to identify the 
coordination spheres or scatterers as well as the nearest neighbor atoms and their distances from the absorbing atom. In this study, EXAFS was used to determine the position of Mn atoms in the crystal lattice of $\mathrm{Bi}_{2-x} \mathrm{Mn}_{x} \mathrm{Se}_{3}$ thin films.

\subsubsection{Hall Effect}

Hall effect is the standard technique to investigate the carrier type, carrier density and mobility in metals and semiconductors. In 1879, Edwin Hall discovered that when a current runs through a conductor in a uniform magnetic field, the charges accumulate on the sides of the sample generating a voltage difference. Measuring this voltage difference enables the determination of electronic properties of the material.

A typical Hall measurement geometry is shown in Fig. 2.12. An electric field is applied with $E_{y}$ causing a current $j_{y}$ in the y direction. The magnetic field $\mathrm{H}$ is applied in out of plane of the sample. If the charges are electrons, they will be deflected in $-x$ direction and accumulates on the sides of the sample due to the Lorentz force [53]

$$
\vec{F}_{L}=\frac{-e}{c} \mathbf{v} \times \mathbf{H} .
$$

Immediately, an electric field is formed to counteract this Lorentz force. Therefore, there is only current $j_{y}$, in y direction in the equilibrium case as shown in Figure 2.12. The magnetoresistance is defined as

$$
\rho=E_{y} / j_{y},
$$

and the Hall coefficient, which relates the transverse electric field to the magnetic field, is written as

$$
R_{H}=\frac{E_{x}}{j_{y} H} .
$$




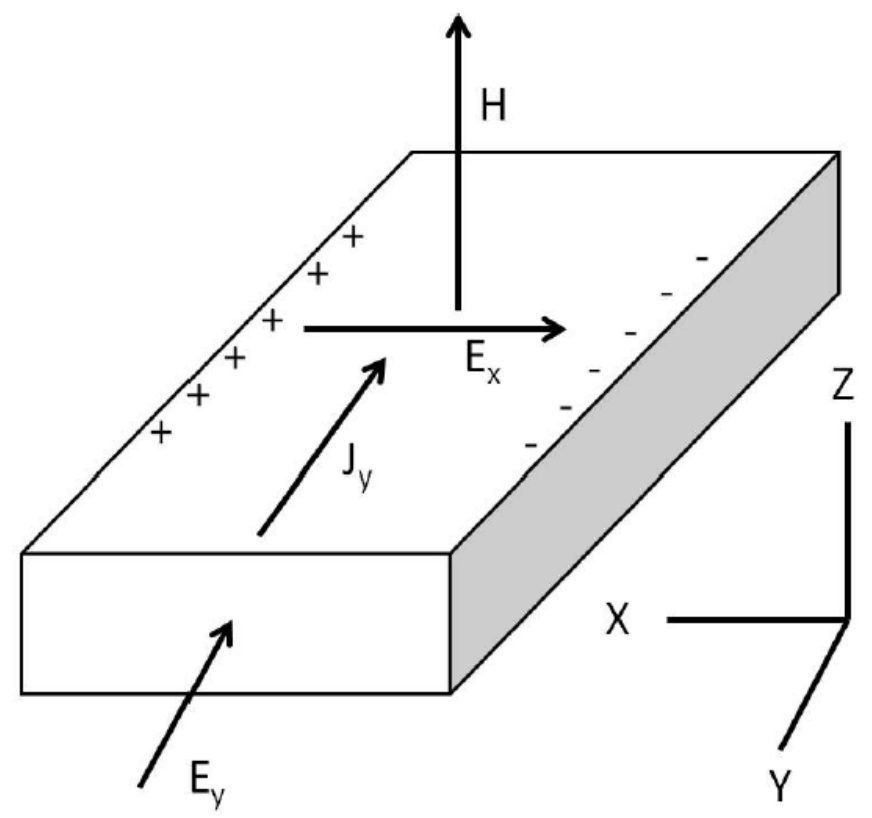

Figure 2.12: Standard geometry for a Hall experiment.

The sign of the Hall coefficient indicates the type of majority of carriers. After a little algebra, one obtains another form of Hall coefficient for the steady state condition,

$$
R_{H}=\frac{1}{n e}
$$

The mobility then becomes

$$
\mu=\frac{1}{\left(\rho R_{H}\right)}
$$

This analysis is used to calculate the type of majority carriers, their density and mobility. The Hall effect was measured for all $\mathrm{Bi}_{2} \mathrm{Se}_{3}$ and $\mathrm{Bi}_{2-x} \mathrm{Mn}_{x} \mathrm{Se}_{3}$ thin films by standard six probe configuration. The carrier density and the mobility were estimated with the procedure mentioned above. Hall measurements were carried out as functions of temperature and magnetic field. 


\section{Chapter 3}

\section{Experimental Methods}

\subsection{Sample Preparation and MBE Growth}

$\mathrm{Al}_{2} \mathrm{O}_{3}$ single crystal substrates of size $10 \times 10 \mathrm{~mm}$ were cleaned in an ultrasonicator for 5 minutes in acetone, methanol and DI water. Prior to the growth, the substrates were annealed in air for 2 hours at $1200{ }^{\circ} \mathrm{C}$ to achieve an atomically smooth surface, as verified by atomic force microscopy. After the annealing process, the substrates were mounted on a $50.8 \mathrm{~mm}$ diameter molybdenum holder with two clips. A load lock chamber with a base pressure of typically $1 \times 10^{-7}$ Torr was used to transfer samples into the vacuum chamber. The load lock chamber was connected to a main transfer chamber which has a base pressure of $\approx 5.0 \times 10^{-9}$ Torr . This transfer chamber was connected to three other UHV chambers which are dedicated to the growth of different materials, such as topological insulators (TIs), transition metal fluorides and oxides. Next, the samples were transferred to the TIs chamber with the help of a mechanical transfer arm. During the growth, Bi (99.999\%), Se (99.999\%) and Mn (99.999\%) were simultaneously evaporated using commercial Knudsen cells. Material fluxes of the constituent Bi, Se and Mn were measured using 
an Inficon quartz crystal microbalance (QCM) controlled via an Inficon IC5 deposition controller placed at the position of the sample. The growth rate was determined by the Bi flux and kept at $0.6 \mathrm{QL} / \mathrm{min}$. The typical flux ranges for materials were, $\mathrm{Bi}$ $\approx 1 \times 10^{16} \mathrm{Se} \approx 1.5 \times 10^{17}$, and $\mathrm{Mn} \approx 1 \times 10^{14} \mathrm{atoms} / \mathrm{cm}^{2}$. The growth was performed in two steps [54]: the first 3 QLs were grown at a substrate temperature of $T_{s}=140$ ${ }^{\circ} \mathrm{C}$, while the subsequent ones after the substrate temperature was raised to $T_{s}=275$ ${ }^{\circ} \mathrm{C}$. The film surface quality was monitored in-situ using RHEED. The typical Se/Bi molar flux ratio value was $15 \pm 0.7$ [55]. After the growth of TI layer, the heater was turned off and the sample was left in the chamber to cool down for a day. To avoid oxidation due to exposure to air, all samples were transferred to another growth chamber without breaking vacuum where they were capped with $10 \mathrm{~nm}$ polycrystalline $\mathrm{MgF}_{2}$ layer grown at room temperature by electron beam evaporation [56]. When growing $\mathrm{Bi}_{2} \mathrm{Se}_{3}$, the thickness was varied, whereas for $\mathrm{Bi}_{2-x} \mathrm{Mn}_{x} \mathrm{Se}_{3}$, the film thickness was fixed and Mn concentration was varied. For undoped samples, films were grown with a thickness range of 6-50 QLs. Another batch of pure samples were grown with a thickness of 12,16, 20, 25 QLs to study the parallel magnetotransport in $\mathrm{Bi}_{2} \mathrm{Se}_{3}$ films. On the other hand, for $\mathrm{Mn}$ doped $\mathrm{Bi}_{2-x} \mathrm{Mn}_{x} \mathrm{Se}_{3}$ films, thickness was fixed to $30 \mathrm{QL}$ and $12 \mathrm{QL}$ in two batch of samples with $x$ values for Mn concentration kept in range $0<x<0.2$.

\subsection{Structural Characterization}

\subsubsection{Reflection High Energy Electron Diffraction (RHEED)}

All RHEED data was taken by using a k-space association data analysis software and an electron beam source from Staib. Corresponding beam voltage and 

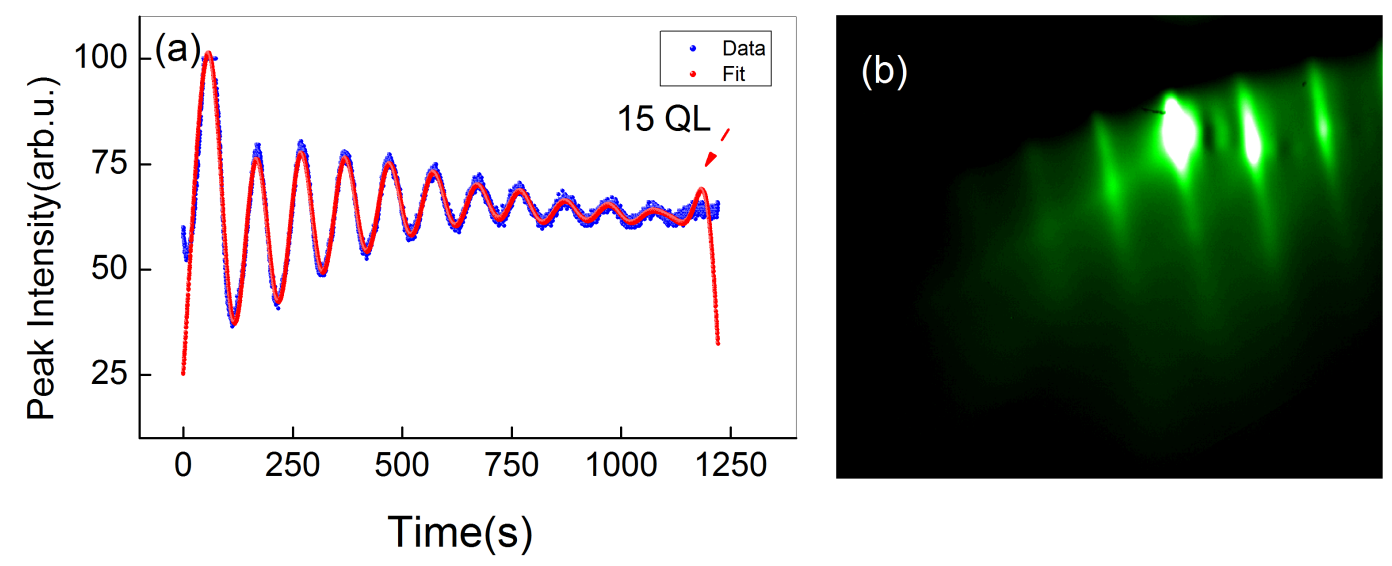

Figure 3.1: (a) RHEED oscillations for a $15 \mathrm{QL} \mathrm{Bi}_{2} \mathrm{Se}_{3}$ thin film. Each peaks corresponds to a completion of a quintuple layer. Note: First 3 QL layers are not shown in the figure. Blue and red circles represent the data and the fit, respectively. (b) Vertical streaks in RHEED image indicating a high quality, smooth surface.

currents were $18 \mathrm{keV}$ and 2.7 A successively. RHEED images of each substrate were taken prior to the film growth. The single crystalline structure of the substrate was confirmed for all samples. During the growth of $\mathrm{Bi}_{2} \mathrm{Se}_{3}$, RHEED oscillations were recorded as function of time. Typical RHEED oscillations and the corresponding RHEED pattern are shown in Fig. 3.1 for a 15 QL thin film. Oscillations corresponding to the first $3 \mathrm{QL}$ layer of $\mathrm{Bi}_{2} \mathrm{Se}_{3}$ were not captured due to the low growth temperatures. Another important finding of RHEED oscillations is the confirmation of film thickness (15 QL) by observing a single oscillation for each quintuple layer (Fig. 3.1a). The streaky RHEED images indicated a high quality, epitaxial film growth (Fig. 3.1b). Although RHEED oscillations were observed during the growth of pure $\mathrm{Bi}_{2} \mathrm{Se}_{3}$ films, indicating quintuple layer-by-layer growth, oscillations were not observed in Mn doped samples, $\mathrm{Bi}_{2-x} \mathrm{Mn}_{x} \mathrm{Se}_{3}$ with $x>0$. A similar RHEED analysis were performed for the $\mathrm{MgF}_{2}$ capping layer and its polycrystalline nature was confirmed. 

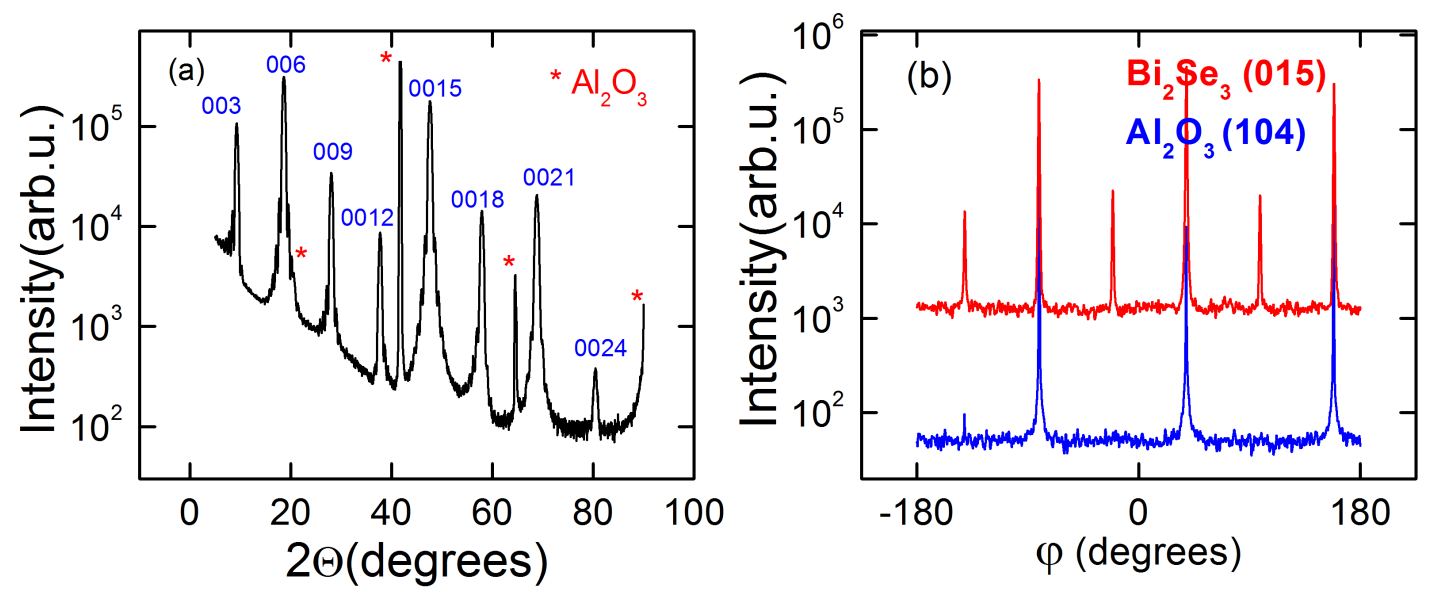

Figure 3.2: (a) X-ray diffraction (XRD) data for $15 \mathrm{QL} \mathrm{Bi}_{2} \mathrm{Se}_{3}$ thin film. Out of plane-(00l) peaks are labeled in the figure. Asterisks represent the peaks from substrate. (b) In-plane x-ray diffraction scan showing three and six peaks for $\mathrm{Al}_{2} \mathrm{O}_{3}$ (104) and $\mathrm{Bi}_{2} \mathrm{Se}_{3}$ (015) reflections, respectively.

\subsubsection{X-ray Diffraction (XRD)}

X-ray diffraction measurements were performed with a Rigaku RU-300 x-ray generator equipped with a Huber four circle diffractrometer using $\mathrm{CuK} \alpha$ radiation source with a wavelength of $1.5418 \AA$. X-rays were alligned with respect to the $\mathrm{Al}_{2} \mathrm{O}_{3}(006)$ and $\mathrm{Bi}_{2} \mathrm{Se}_{3}(0015)$ peaks. The out of plane $\theta-2 \theta$ scans were perfomed on each thin film to identify the growth direction and corresponding phases. The c-axis lattice parameter (using the hexagonal crystal structure basis) was calculated from out-of-plane (q momentum transfer vector perpendicular to the plane of the sample) x-ray measurements. Rocking curves of $\mathrm{Bi}_{2} \mathrm{Se}_{3}$ (0015) peak were measured to compare the quality of films relative to each other. Single crystal ordering was confirmed by observing (00l) family of planes (in the hexagonal basis) for all $\mathrm{Bi}_{2} \mathrm{Se}_{3}$ and $\mathrm{Bi}_{2-x} \mathrm{Mn}_{x} \mathrm{Se}_{3}$ thin films. In-plane XRD measurements were performed to check the epitaxial relation between the $\mathrm{Al}_{2} \mathrm{O}_{3}(104)$ and $\mathrm{Bi}_{2} \mathrm{Se}_{3}$ (015) peaks. The epitaxial relation between the film and the substrate was established with an additional twin 
domain formation. The in-plane lattice parameter $a$ was calculated by using in and out of plane x-ray diffraction data. An example of an XRD pattern for a $15 \mathrm{QL}$ sample is shown in Fig. 3.2. The rhombohedrally layered structure of $\mathrm{Bi}_{2} \mathrm{Se}_{3}$ was confirmed with the observation of $(00 l)$ peaks (Fig. 3.2a). No secondary phases were detected in this scans. Azimuthal XRD scans indicated a twin domain in $\mathrm{Bi}_{2} \mathrm{Se}_{3}$ growth for the $\mathrm{Bi}_{2} \mathrm{Se}_{3}(015)$ reflection $[57,58]$ (Fig. 3.2(b)). The out of plane (00l) ordering and the formation of twins were observed for all the samples in this study.

\subsubsection{X-ray Reflectivity (XRR)}

X-ray reflectivity measurements were performed with the same Rigaku RU-300 x-ray generator. Two circle diffractometer was used for reflectivity measurements with a resolution of $0.003 \AA^{-1}$. The data was analyzed using open source software GenX [59] based on Parratt's recursion algorithm mentioned in Chapter 2. The thickness and roughnesses of all layers were estimated by GenX fits. Figure 3.3 shows the models used in determining the layer thickness and roughness for $\mathrm{Bi}_{2} \mathrm{Se}_{3}$ and $\mathrm{Bi}_{2-x} \mathrm{Mn}_{x} \mathrm{Se}_{3}$ thin films. We used two different models to fit our data. The first one involved the fitting in the high $Q$ range including the first Bragg peak (003) while the second model was only used in low $Q$ range without the Bragg peak. For all samples, an interface layer at the top and/or bottom consisting of the average

electron density of the substrate and $\mathrm{Bi}_{2} \mathrm{Se}_{3}$ (for the bottom) or of $\mathrm{MgF}_{2}$ and $\mathrm{Bi}_{2} \mathrm{Se}_{3}$ (for the top) was included in order to simulate larger interface disorder parameters. Thickness and roughness were estimated from the scattering length density (SLD) and its derivative profiles [60]. An example of XRR data and fit can be seen in figure 3.4 for our $15 \mathrm{QL} \mathrm{Bi}_{2} \mathrm{Se}_{3}$ sample. The GenX fits to the XRR data looks in excellent agreement. The sample thickness was estimated to be $13.95 \mathrm{~nm}$ for this 15 

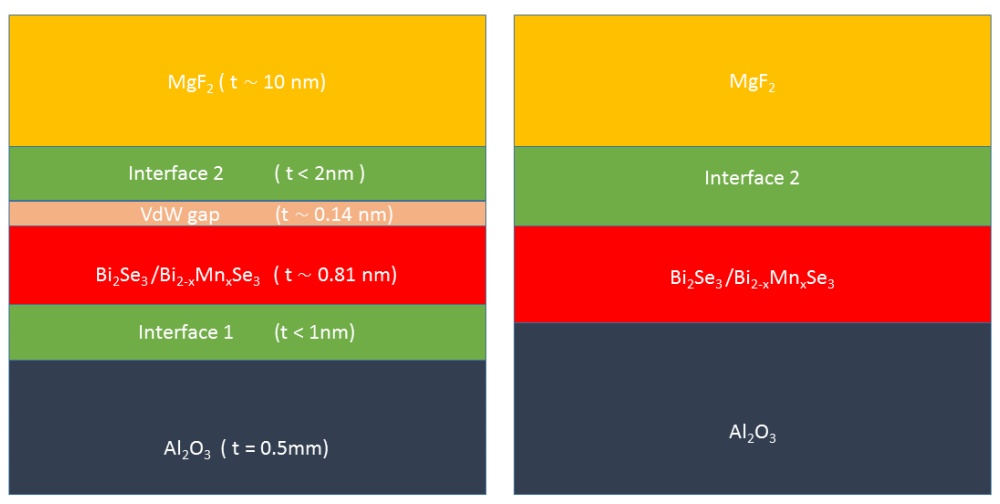

Figure 3.3: XRR fit models for $\mathrm{Bi}_{2} \mathrm{Se}_{3}$ and $\mathrm{Bi}_{2-x} \mathrm{Mn}_{x} \mathrm{Se}_{3}$ thin films. Approximate thickness of each layer are labeled (left) for the first model which was used in high $Q$ range. The model seen on the right was used in low $Q$ range.

QL sample. Similarly, the thickness of $\mathrm{MgF}_{2}$ layer was $11.5 \mathrm{~nm}$ (Fig. 3.4b). The roughness values were estimated from the width of the first derivatives of SLD with the values of $0.17 \mathrm{~nm}$ and $1.47 \mathrm{~nm}$ for $\mathrm{Bi}_{2} \mathrm{Se}_{3}$ and $\mathrm{MgF}_{2}$ layers, respectively (Fig. $3.4 c)$.

\subsubsection{Atomic Force Microscopy (AFM)}

All AFM measurements were performed in tapping mode using Nanoscope IIIa controller. AFM centilavers were $125 \mu \mathrm{m}$ long, $2.1 \mu \mathrm{m}$ thick and had a radius of curvature less than $8 \mathrm{~nm}$ with resonance frequency of $160 \mathrm{kHz}$. Due to the capping layer of $\mathrm{MgF}_{2}$, interpretation of the AFM data involved consideration of both capping and the underlying $\mathrm{Bi}_{2} \mathrm{Se}_{3}$ layers. Figure 3.5 shows a typical surface topography of $\mathrm{MgF}_{2} / \mathrm{Bi}_{2} \mathrm{Se}_{3}$ layer on sapphire substrate. The triangular features are signatures of dislocations present in $\mathrm{Bi}_{2} \mathrm{Se}_{3}$ layer. The roughness of $\mathrm{MgF}_{2} / \mathrm{Bi}_{2} \mathrm{Se}_{3}$ layer was obtained from Nanoscope software program Nanoscope 5.31, which yielded a value of $0.9 \mathrm{~nm}$. 

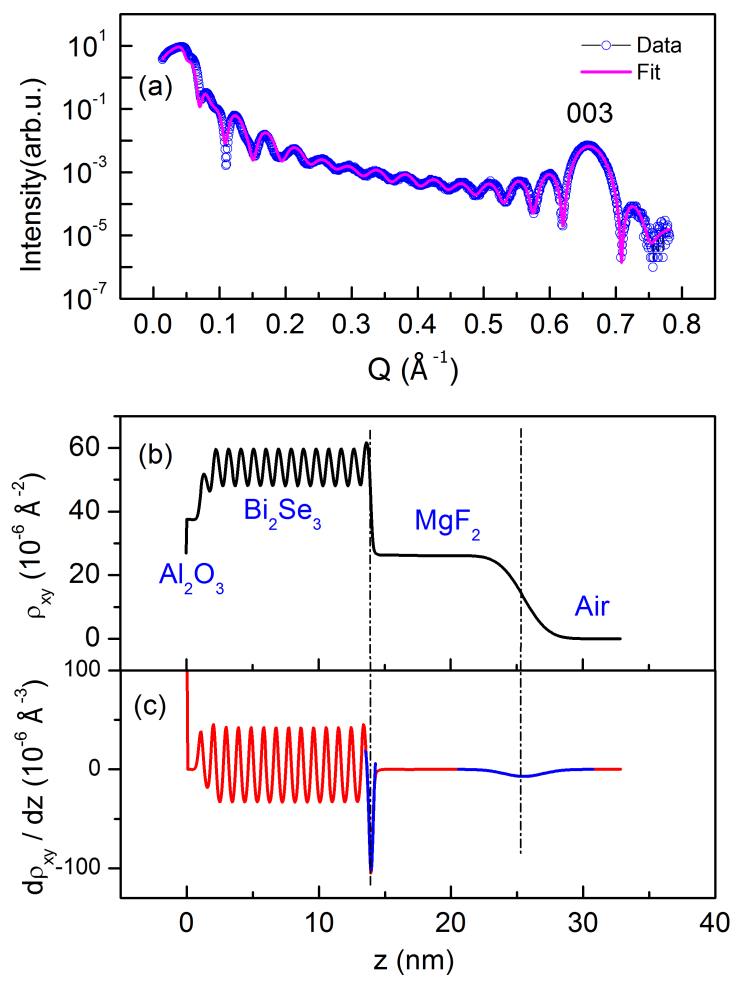

Figure 3.4: (a) X-ray reflectivity data and the fit for a $15 \mathrm{QL} \mathrm{Bi}_{2} \mathrm{Se}_{3}$ thin film. Blue circles and magenta line represent the data and the fit, respectively. The first Bragg peak of $\mathrm{Bi}_{2} \mathrm{Se}_{3}$ (003) labeled in figure at $Q=0.65 \AA^{-1}$. (b) SLD profiles extracted from the GenX fits with names of each layer. (c) First derivative of SLD data. Blue curves represents the Gaussian fits to obtain roughness values of $\mathrm{Bi}_{2} \mathrm{Se}_{3}$ and $\mathrm{MgF}_{2}$ layers.

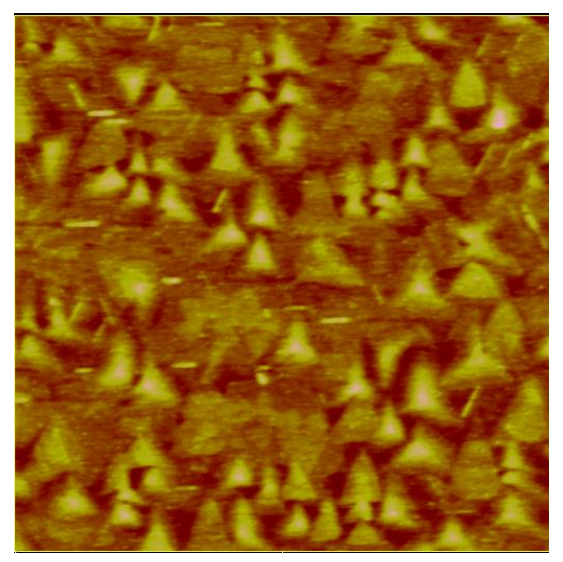

Figure 3.5: 2 x $2 \mu \mathrm{m}^{2}$, ex-situ AFM image of $15 \mathrm{QL} \mathrm{Bi}_{2} \mathrm{Se}_{3}$ film capped with a $\mathrm{MgF}_{2}$ layer. Estimated film roughness is $0.9 \mathrm{~nm}$. 


\subsubsection{X-ray Photoelectron Spectroscopy (XPS)}

XPS measurements were performed in order to confirm the nominal doping concentrations of $\mathrm{Mn}$ in $\mathrm{Bi}_{2-x} \mathrm{Mn}_{x} \mathrm{Se}_{3}$ thin films and also to determine the valency of $\mathrm{Mn}$ impurities. XPS depth profile analysis was performed using a Physical Electronics PHI 5000 Versa Probe XPS system with a monochromatic Al K $\alpha$ source (energy = $1486.7 \mathrm{eV}$ ) and a beam diameter of approximately $100 \mu \mathrm{m}$ equipped with a hemispherical detector oriented at a $45^{0}$ take-off angle withrespect to the sample surface. The $\mathrm{MgF}_{2}$ capping layer was sputtered off using Ar ions with an energy of $2 \mathrm{keV}$ and a current of $10 \mu \mathrm{A}$ for $6 \mathrm{~s}$ intervals. Between each interval, survey scans from 0 $\mathrm{eV}$ to $1400 \mathrm{eV}$ were performed. This was repeated until the $\mathrm{Mg}$ 1s and $\mathrm{F}$ 1s peaks almost disappeared. Once this was done, detailed scans of the Bi 4f, Se 3d, and Mn $2 p$ transitions were acquired. The binding energies were calibrated to the $\mathrm{C} 1 \mathrm{~s}$ peak coming from the surface of the sample before sputtering.

\subsubsection{Extended X-ray Absorption Fine Structure (EXAFS)}

In order to characterize the Mn impurity sites, EXAFS measurement was performed on a $30 \mathrm{QL} \mathrm{Bi}_{2-x} \mathrm{Mn}_{x} \mathrm{Se}_{3}$ sample with $x=0.063$. EXAFS data were taken at Advanced Light Source (ALS) beamline 10.3.2 at the Mn Kedge [61]. The sample was set at a grazing angle to the beam of $2^{0}$ in order to increase the signal from the thin $\mathrm{Bi}_{2-x} \mathrm{Mn}_{x} \mathrm{Se}_{3}$ layer. The beam spot in the horizontal was $12 \mu \mathrm{m}$, making the footprint on the sample $350 \mu \mathrm{m}$ wide. The fluorescence signal was detected using an AmpTek drift-diode detector. The data were taken out to $k=11.4 \AA^{-1}$. The data were analyzed using Artemis software. 


\subsection{Electrical Transport}

Electrical transport measurements were performed in a PPMS EverCool-II system from Quantum Design with a magnetic field capability of 9 Tesla. The measurements were performed on both pure and Mn doped films. Six point Hall bar devices with well defined dimensions were patterned by photolithography process. The dimensions of the devices were $l \times w=1.37 \times 0.50 \mathrm{~mm}$ as shown in Fig. 3.6. After patterning and etching process of the films, Ag paste was used to form the electrical leads at each corner of the $1 \mathrm{~mm}$ size squares. Samples were mounted on a PPMS resistivity puck, and copper wires used to form electrical connections between the sample and the resistivity puck. An excitation current of $1 \mu \mathrm{A}$ was applied between current leads in the transport measurements. Resistivity vs. temperature was measured in zero and non-zero magnetic fields. Carrier transport measurements were performed with a minimum temperature of $T=2 \mathrm{~K}$. The sequences for the transport measurements were written in the PPMS EverCool-II software. During temperature dependent resistivity measurements, temperature was swept continuously, whereas during field dependent measurements, magnetic field was kept in persistent mode and the corresponding data was recorded in excel sheets. 


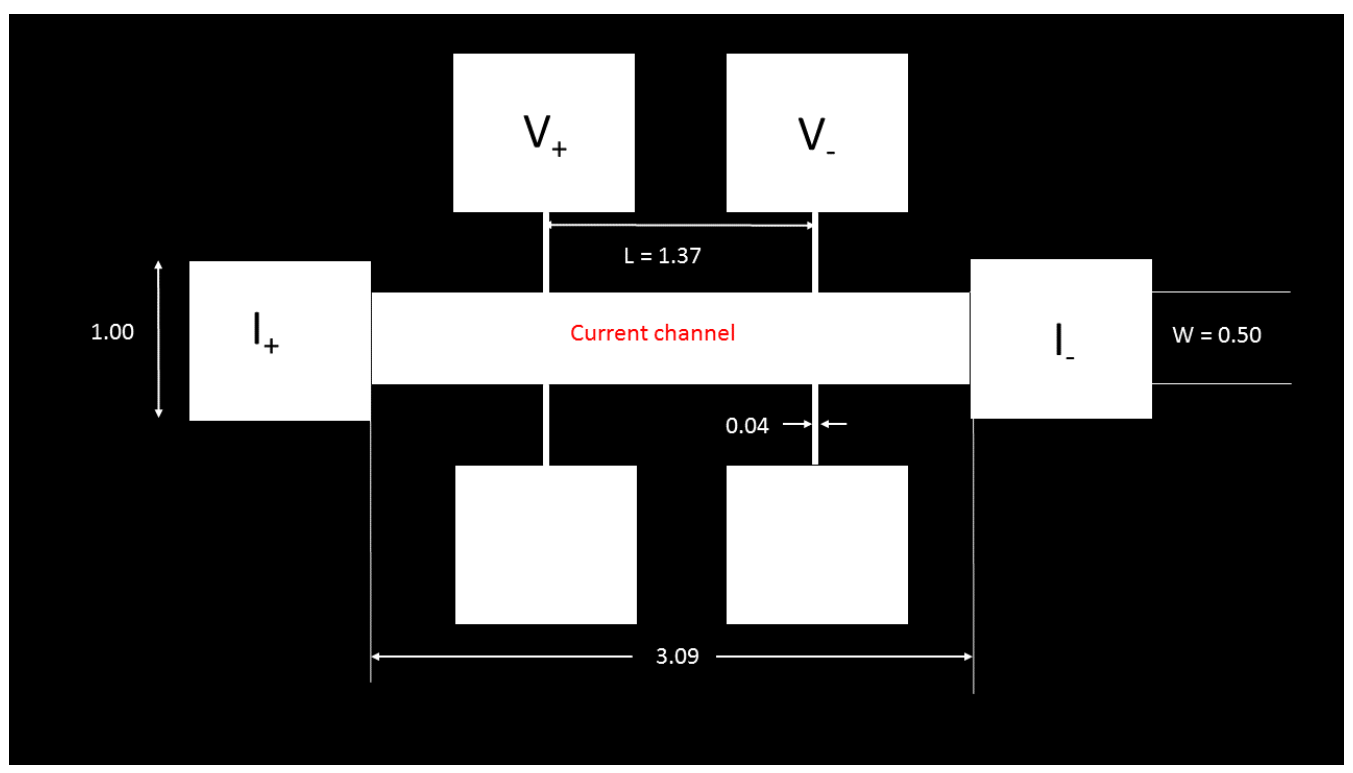

Figure 3.6: Dimensions of six contact Hall bar used in transport measurements. All units are in $\mathrm{mm}$. 


\section{Chapter 4}

\section{Structural Properties of}

\section{$\mathrm{Bi}_{2-x} \mathrm{Mn}_{x} \mathrm{Se}_{3}$ Thin Films Grown Via Molecular Beam Epitaxy}

\subsection{Introduction}

The effect of magnetic impurity doping in TIs have been the focus of several studies in nanoribbons, thin films and single crystals [62-65]. In some cases, WAL - WL transition is observed with the increasing doping concentration [66]. Another interesting paper discusses the carrier type conversion in $\mathrm{Mn}$ doped $\mathrm{Bi}_{2} \mathrm{Se}_{3}$ single crystals [67]. The goal of this chapter is to understand the structural changes in $\mathrm{Bi}_{2} \mathrm{Se}_{3}$ thin films induced by Mn doping. Determining valence state of Mn via XPS, and EXAFS, and monitoring crystallinity, roughness properties via RHEED, XRD, XRR motivates us to discuss the structural properties of our thin films. Resistivity and Hall effect were also discussed to obtain a relation between the structural and electronic behavior in $\mathrm{Mn}$ doped $\mathrm{Bi}_{2} \mathrm{Se}_{3}$ thin films. Our analysis was published in an 
article by Babakiray et al. 2015, in reference [68].

We report on the structural properties of $30 \mathrm{QL}$ thick $\mathrm{Mn}$ doped $\mathrm{Bi}_{2} \mathrm{Se}_{3}$ thin films grown by molecular beam epitaxy (MBE). X-ray photoelectron spectroscopy (XPS) data indicate that $\mathrm{Mn}$ is incorporated into the lattice with a valency similar to that of MnSe (2+). Using extended x-ray absorption fine structure (EXAFS) measurements, we show that the $\mathrm{Mn}$ is incorporated substitutionally into the Bi sites and that the distance to Se nearest neighbors is significantly smaller than the Bi-Se interatomic

distance. X-ray diffraction (XRD) data indicate that a lattice expansion along the [001] direction parallel to the growth direction, as a function of Mn concentration, also observed in Cr-doped $\mathrm{Bi}_{2} \mathrm{Se}_{3}$ thin films, is accompanied by a decrease in the lattice constant in the plane of the sample. These findings are consistent with the EXAFS data if Mn doping weakens the van der Waals bonding between adjacent Se planes. The changes in the carrier density and Hall mobility at low temperature as functions of Mn concentration are consistent with the structural characterization results, including the Mn valency.

\subsection{Growth and Structural Characterization}

$\mathrm{Bi}_{2-x} \mathrm{Mn}_{x} \mathrm{Se}_{3}$ thin films with a thickness of $30 \mathrm{QL}$ and $x=0,0.024,0.047,0.063$ were grown by MBE. The film surface quality was monitored in-situ using RHEED. In-situ RHEED patterns were taken after the growth finished at $T_{s}=275^{\circ} \mathrm{C}$. RHEED data indicated an increasing disorder with Mn concentration and showed transmission spots in the highly doped $x=0.063$ sample. Although increasing Mn concentration caused a degradation of the surface quality, streaky RHEED patterns confirmed the formation of an epitaxial film with a relatively smooth surface (See Fig. 4.1).

AFM images of surface topography obtained from the top of the corresponding 

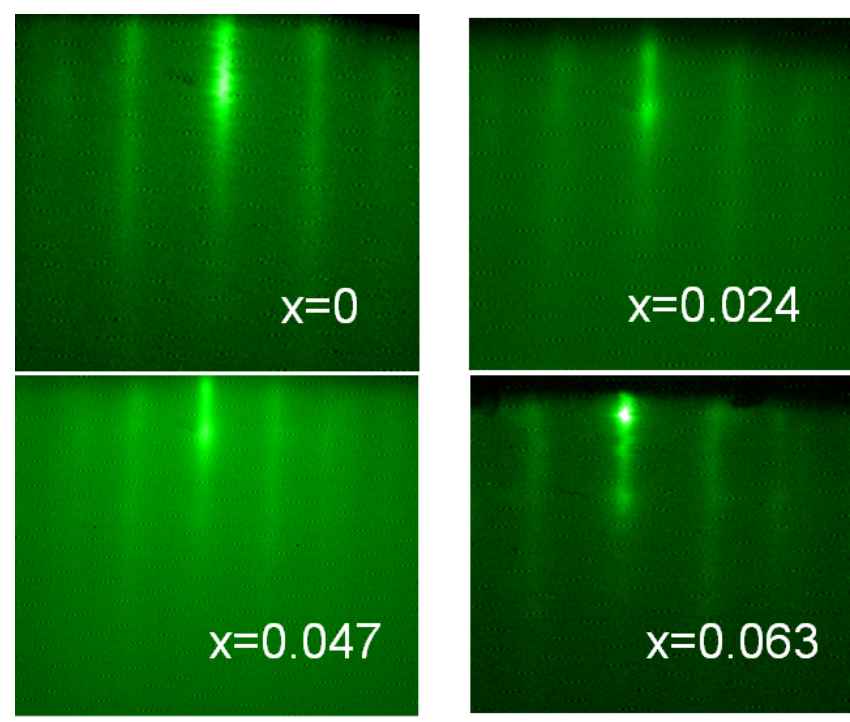

Figure 4.1: RHEED pattern for $30 \mathrm{QL} \mathrm{Bi}_{2-x} \mathrm{Mn}_{x} \mathrm{Se}_{3}$ films taken prior to capping by $\mathrm{MgF}_{2}$. Rough sample surface was observed in highly doped samples, especially for $x=0.063$ sample.

$\mathrm{MgF}_{2}$ capping layers had triangular structures for all samples which are likely related to the underlying $\mathrm{Bi}_{2-x} \mathrm{Mn}_{x} \mathrm{Se}_{3}$. AFM images of all the samples are shown in Fig. 4.2. The RMS surface roughnesses were obtained from Nanoscope software program Nanoscope 5.31, with the values of $2.2,2.8,3.8$, and $2.8 \mathrm{~nm}$ for $x=0,0.024,0.047$ and 0.063, respectively. Figure 4.3 shows the results of the XRR measurements for all of our samples. For the samples with higher Mn concentrations, the interface roughness at the $\mathrm{Bi}_{2-x} \mathrm{Mn}_{x} \mathrm{Se}_{3} / \mathrm{MgF}_{2}$ interface was larger, so in order to model this an interface layer between the $\mathrm{Bi}_{2-x} \mathrm{Mn}_{x} \mathrm{Se}_{3}$ and $\mathrm{MgF}_{2}$ layers with a scattering coherence length equal to the average of the two materials was introduced. The fits to the data yielded $\mathrm{Bi}_{2-x} \mathrm{Mn}_{x} \mathrm{Se}_{3}$ film thickness values of $31.5,30.7,29.3,28.5 \pm 0.2 \mathrm{~nm}$ with interface layer roughness of $1.9,4.6,4.8,5.2 \pm 0.1 \mathrm{~nm}$ for the $x=0,0.024,0.047,0.063$ samples, respectively (Fig. 4.3 and inset). This is indicative of the increasing roughness as a function of increasing $\mathrm{Mn}$ concentration. The roughness obtained from the fits shown in Fig. 4.3 between the $\mathrm{Bi}_{2-x} \mathrm{Mn}_{x} \mathrm{Se}_{3}$ and interface layer are plotted in the 

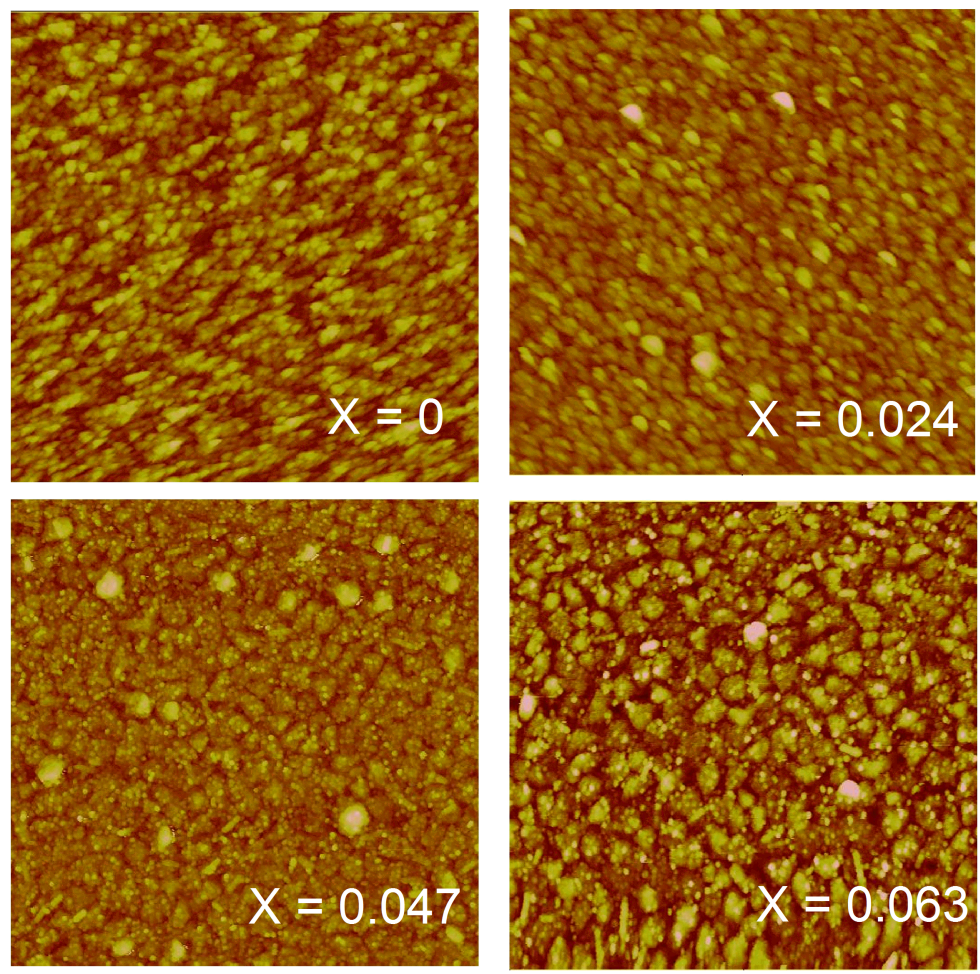

Figure 4.2: Ex-situ AFM images $(2 \mu \mathrm{m} \times 2 \mu \mathrm{m})$ of the $\mathrm{Bi}_{2-x} \mathrm{Mn}_{x} \mathrm{Se}_{3}$ films with different $x$ values.

inset to Fig. 4.3. This is another way of quantitatively characterizing the increase of the surface roughness of the $\mathrm{Bi}_{2-x} \mathrm{Mn}_{x} \mathrm{Se}_{3}$ films with increasing $\mathrm{x}$. These roughness values were consistent with the RMS roughness obtained from the AFM data.

XRD patterns for all samples are presented in Fig. 4.4a. The films were [001]oriented and the calculated out-of-plane hexagonal lattice parameter, c, increased (Fig. 4.4c) while the in-plane lattice constant, a, (Fig. 4.4d) decreased with increasing $\mathrm{x}$. The value of c was determined from the out-of-plane (001) peaks (hexagonal basis) while the value of a was determined from the position of the (015) peak and the value of c. A representative in-plane XRD scan for the $x=0.047$ is shown in Fig. 4.4b for $\mathrm{Bi}_{2} \mathrm{Se}_{3}$ (015) reflection. Similar scans were performed for all other films. A similar increase in c, with increasing $\mathrm{Cr}$ impurity concentration has been observed 


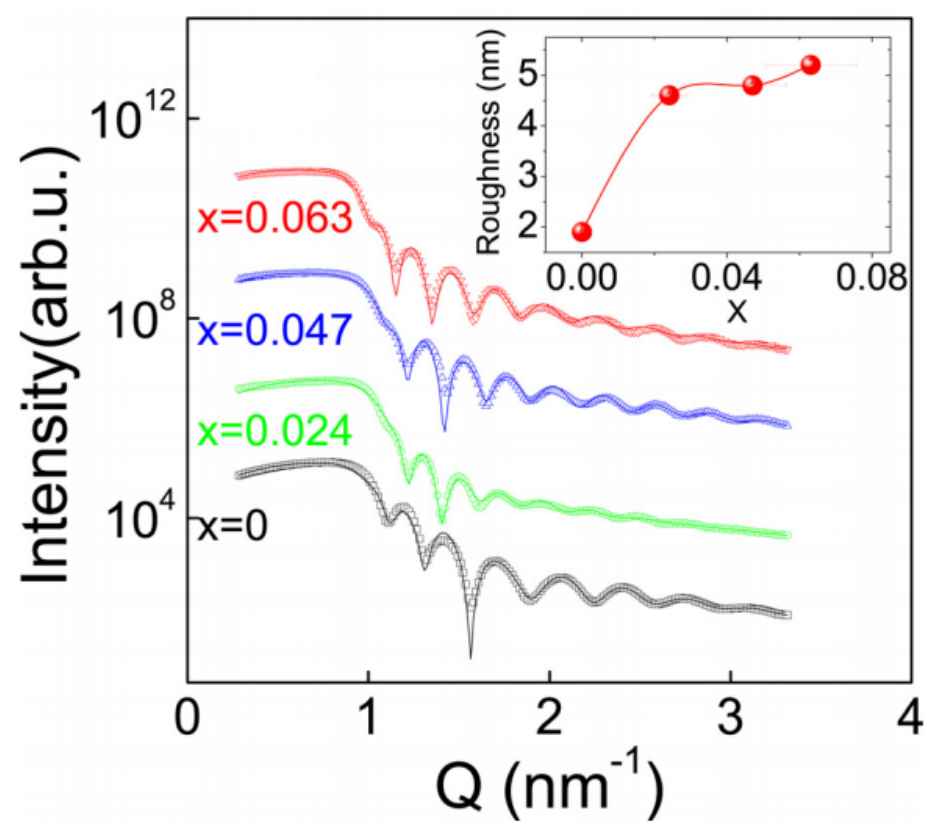

Figure 4.3: Low angle x-ray reflectivity data for the $\mathrm{Bi}_{2-x} \mathrm{Mn}_{x} \mathrm{Se}_{3}$ films as a function of momentum transfer $Q$. Circles are the data and solid curves are corresponding fits. Inset: RMS roughness at the $\mathrm{Bi}_{2-x} \mathrm{Mn}_{x} \mathrm{Se}_{3} / \mathrm{MgF}_{2}$ interface obtained from fits to the XRR data for the samples used in this study. Curve is a guide to the eye.

in $\mathrm{Bi}_{2-x} \mathrm{Cr}_{x} \mathrm{Se}_{3}$ thin films [64], where the increase in c was attributed to intercalated Cr. As discussed in more detail below, however, intercalation of Mn in the Mn-doped samples is unlikely because of the simultaneous decrease in a and the analysis of the EXAFS data described below.

To investigate the structural disorder in the films, rocking curve scans of the (00.15) peak were performed. The rocking curves are shown in Fig.4.5 and the calculated full width at half maximum (FWHM) are indicated in the figure. Because the rocking curve widths were larger in the Mn-doped samples in comparison to the pure sample, we conclude that crystallographic disorder increased with increasing Mn impurity level. Other samples grown with $x>0.063$ were not epitaxial; indeed, it was not possible to obtain RHEED or x-ray diffraction patterns for these samples which indicated that they were amorphous or polycrystalline. This trend is consistent 

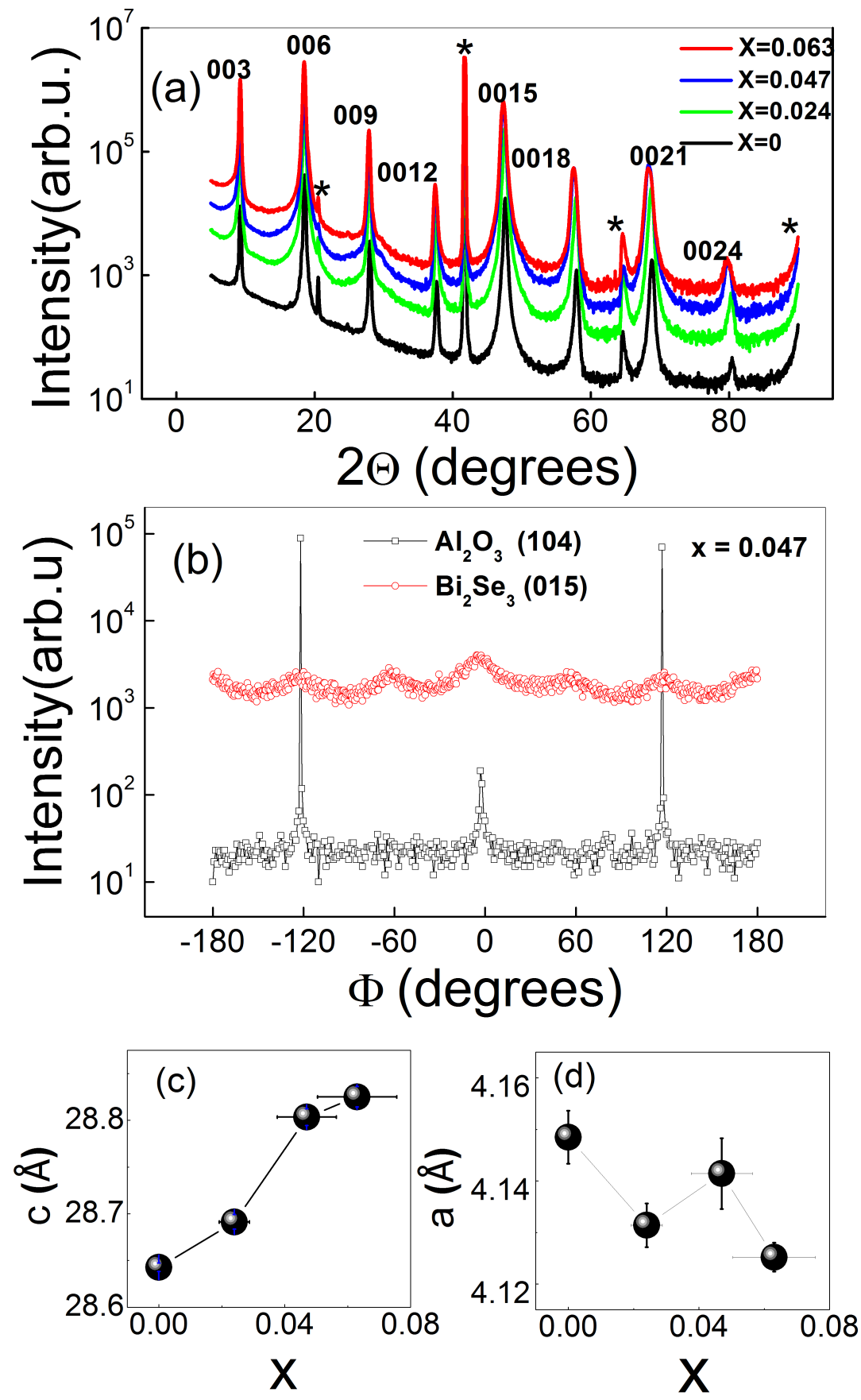

Figure 4.4: High angle XRD scan for $\mathrm{Bi}_{2-x} \mathrm{Mn}_{x} \mathrm{Se}_{3} / \mathrm{Al}_{2} \mathrm{O}_{3}$ samples. The (001) peaks are indicated. Asterisks indicate substrate peaks. (b) In-plane azimuthal XRD scan for $x=0.047$ sample. Six reflections from the $\mathrm{Bi}_{2} \mathrm{Se}_{3}$ (015) peak confirms the formation of twins. (c) Out-of-plane c-axis lattice constant as a function of x. (d) In-plane a-axis lattice constant as a function of $\mathrm{x}$. 

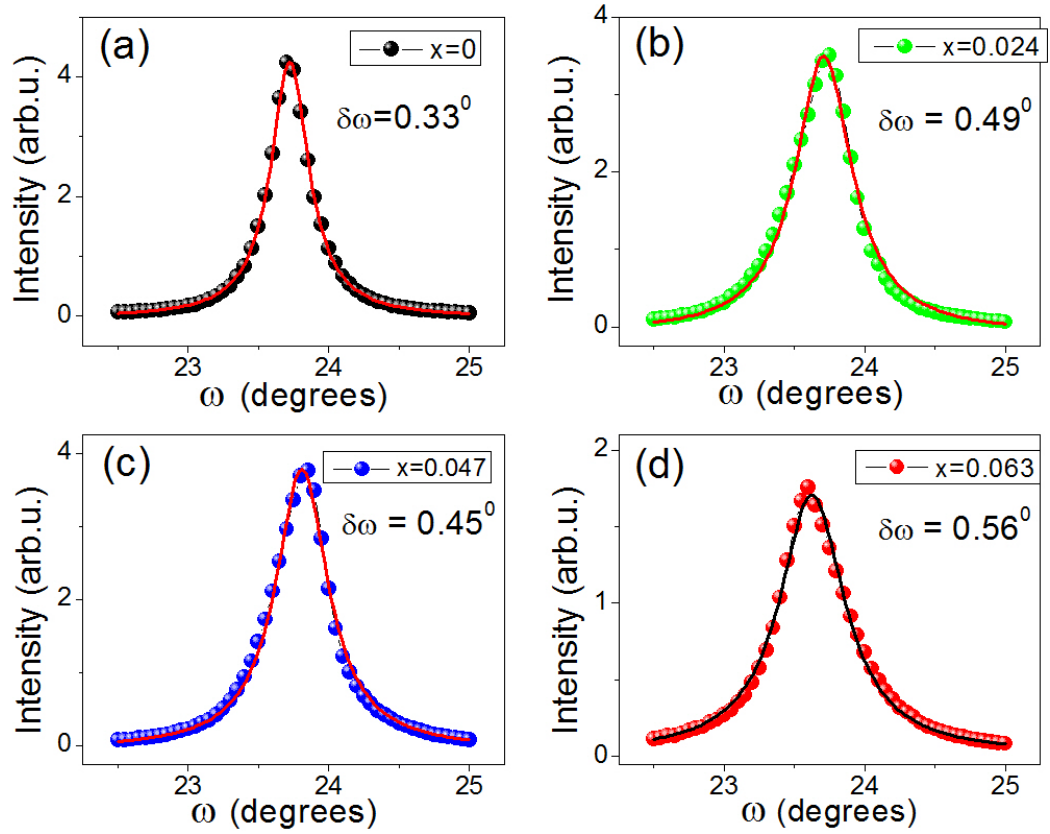

Figure 4.5: Rocking curves of (00.15) peak of $\mathrm{Bi}_{2} \mathrm{Se}_{3}$ for samples with different $\mathrm{Mn}$ concentrations $\mathrm{x}$. The solid curves are fits to a Gaussian peak function. The full widths at half maximum values $(\delta \omega)$ determined from the fits are indicated in the figure.

with other work on Mn-doped $\mathrm{Bi}_{2} \mathrm{Se}_{3}$ [69] and with measurements of $\mathrm{Bi}_{2-x} \mathrm{Cr}_{x} \mathrm{Se}_{3}$ thin films, where the crystalline structure quickly deteriorated with increasing $\mathrm{Cr}$ doping [64].

\subsubsection{XPS Results}

XPS measurements were performed on a $12 \mathrm{QL} \mathrm{Bi}_{2-x} \mathrm{Mn}_{x} \mathrm{Se}_{3}$ with $x=0.13$ grown under the same conditions as other samples. This sample was used to calibrate the nominal doping concentrations and to determine the valency of Mn impurities. In order to have a large signal from Mn impurities, the doping concentration was chosen to be $x=0.13$ with a sample thickness of $12 \mathrm{QL}$ which preserved the single phase of $\mathrm{Bi}_{2} \mathrm{Se}_{3}$. The corresponding XPS peaks of Bi-4f, Se-3d and Mn-2p are shown in 


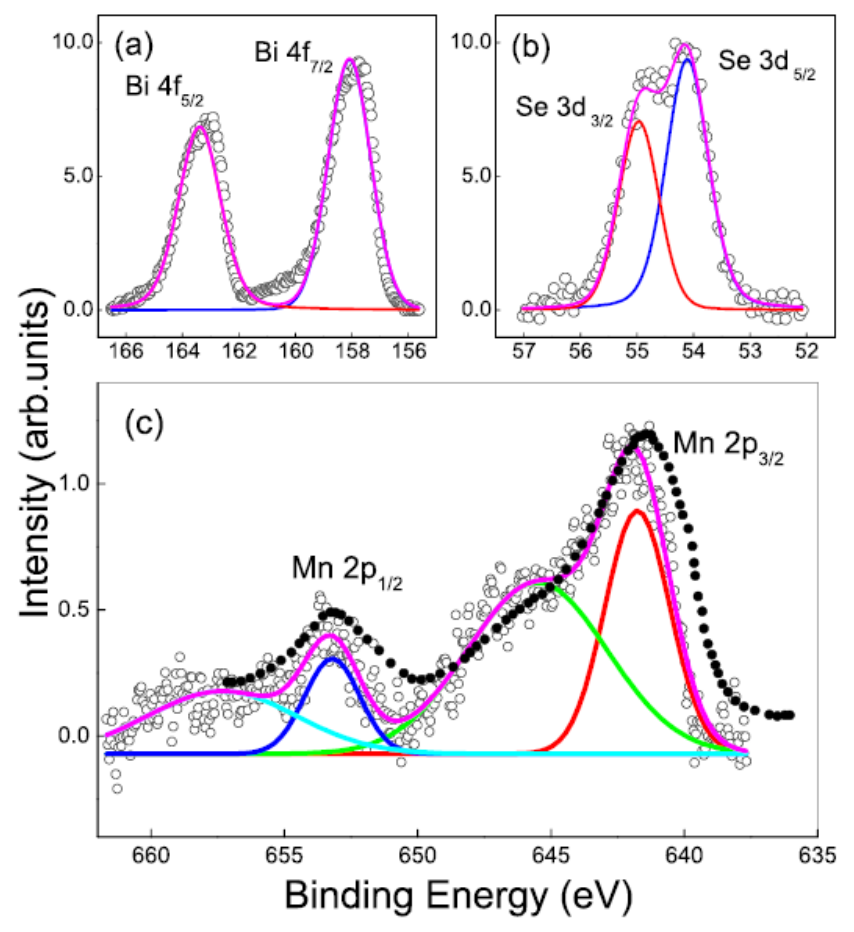

Figure 4.6: XPS scans of Bi-4f (a), Se-3d (b) and Mn-2p (c) core levels for the 12 QL thick $x=0.13$ sample. Data were acquired after sputtering off the $\mathrm{MgF}_{2}$ capping layer as described in the text. The magenta curves represent the fit to Gaussian lineshapes which are shown by the red, blue, and green curves. The red and blue curves represent the electron spectra contributions of $4 f_{5 / 2}$ and $4 f_{7 / 2}$ for $\mathrm{Bi}$, the $3 d_{3 / 2}$ and $3 d_{5 / 2}$ for Se, and $2 p_{3 / 2}$ and $2 p_{1 / 2}$ for Mn, respectively. For Mn (c), satellite peak contributions are also shown by the green and light blue curves. The black dots represent data for MnSe powder reported in Ref. [70].

Fig. 4.6. The peaks arising from different transitions were fit to Gaussian lineshapes, with the total contributions of all transitions (background subtracted) shown by the magenta curves in Fig. 4.6. The center of the peaks (binding energies EB) and widths of the peaks determined from the fits are shown in Table 6.2. Atomic concentrations of Mn were calculated by comparing the area under the curves of the Bi (Fig. 4.6(a)) and Mn (Fig. 4.6(c)) signals, and the calibration of the quartz crystal monitor was confirmed to be accurate to better than $20 \%$. Bi 4 f and Se 3d peaks were split due to spin-orbit coupling. The Bi $4 f_{7 / 2}$ and $4 f_{5 / 2}$ were separated with an energy of $5.4 \mathrm{eV}$ 
(Fig. 4.6(a)) while Se $3 d_{5 / 2}$ and $3 d_{3 / 2}$ seperation was $0.9 \mathrm{eV}$ (Fig. 4.6(b)) confirming previous results for $\mathrm{Bi}_{2} \mathrm{Se}_{3}$ cleaved in vacuum [71]. The shoulder arising around $\mathrm{E}_{B} \approx 160 \mathrm{eV}$ between $\mathrm{Bi} 4 \mathrm{f}$ peaks is typically attributed to Se 3p $3 / 2$ [72]. Fig. 4.6(c) shows the multiplet splitting of the Mn-2p spectrum. The red and blue curves in Fig. 4.6(c) are the contributions to the $2 p_{3 / 2}$ and $2 p_{1 / 2}$ peaks, while the green and light blue curves are satellite peaks, resulting mostly from charge transfer effects between ligand $p$ orbitals (in this case, the Se $3 p$ orbitals, assuming bonding with Se nearest neighbors) and metal d orbitals (i.e., Mn 3d orbitals), which are commonly observed in transition-metal compounds [73]. The position of the $2 p_{3 / 2}$ peak at a binding energy $\mathrm{E}_{B} \approx 641.8 \pm 0.1 \mathrm{eV}$ agrees well with the values found in the literature for $\mathrm{Mn}_{2} \mathrm{O}_{3}(641.7 \mathrm{eV})$ and MnSe $(641.8 \mathrm{eV})$. Moreover, the separation between the main $2 p_{3 / 2}$ and $2 p_{1 / 2}$ peaks of $11.5 \mathrm{eV}$, resulting from spin-orbit coupling in the Mn atom, is in excellent agreement with data from Mn compounds. [70,73-75] The presence of broad satellite peaks at $E_{B}=645.5$ and $657.3 \mathrm{eV}$ is considered a reference for the presence of $\mathrm{Mn}^{2+}$, which has been observed in $\mathrm{MnO},[74] \mathrm{MnTe},[76]$ and MnSe, [70] but not in $\mathrm{Mn}_{2} \mathrm{O}_{3}$, [75] which is a $\mathrm{Mn}^{3+}$ compound. In Fig. 4(c), we also plot data for MnSe powder digitized from Ref. [70] (background not removed). Our data is similar to the MnSe data in terms of the positions of the main and satellite Mn $2 \mathrm{p}$ peaks. The shoulder in the MnSe data near $640 \mathrm{eV}$ likely comes from $\mathrm{MnSe}_{2}$ impurities, since the $2 p_{3 / 2}$ peak for $\mathrm{MnSe}_{2}$ is known to occur at $640.5 \mathrm{eV}$. [77] The position of the $\mathrm{Mn}$ peak in our sample at $641.8 \mathrm{eV}$, together with an absence of a shoulder at 640.5, is strong evidence that $\mathrm{Mn}$ in our $\mathrm{Bi}_{2-x} \mathrm{Mn}_{x} \mathrm{Se}_{3}$ is in the $\mathrm{Mn}^{2+}$ state. Other work on XPS measurements of Cr-doped $\mathrm{Bi}_{2} \mathrm{Se}_{3}$ indicates that $\mathrm{Cr}$ impurities also tend to be in the divalent state, in agreement with our results. [78] 


\begin{tabular}{|ccc|}
\hline Core level & $\mathrm{E}_{B}(\mathrm{eV})$ & FWHM $(\mathrm{eV})$ \\
\hline Bi $4 f_{7 / 2}$ & 158.1 & 1.8 \\
Bi $4 f_{5 / 2}$ & 163.4 & 1.5 \\
Se $3 d_{5 / 2}$ & 54.0 & 0.8 \\
Se $3 d_{3 / 2}$ & 54.9 & 1.0 \\
Mn $2 p_{3 / 2}$ & 641.8 & 2.9 \\
(satellite) & 645.5 & 6.2 \\
Mn $2 p_{1 / 2}$ & 653.2 & 2.4 \\
(satellite) & 657.3 & 6.6 \\
\hline
\end{tabular}

Table 4.1: Results of XPS data with corresponding binding energies for each element and FWHM determined from Gaussian lineshapes. The uncertainities is less than \pm $0.1 \mathrm{eV}$. Second peaks under Mn $2 \mathrm{p}$ are the corresponding satellite peaks.

\subsubsection{EXAFS Analysis}

EXAFS measurements were performed on the $\mathrm{x}=0.063$ sample to determine the specific sites in the lattice where the Mn atoms are absorbed. Data were analyzed using the Artemis software [79] to fit the first two shells, with initial geometry derived from the undistorted crystal structure [80]. Fits were done on the real space Fourier transform (FT) of the response $k^{3} \chi(k), \chi(R)$, performed in the range of 2.5 $<k<10.0 \AA^{-1}$, as shown in Fig. 4.7. Three scenarios were considered: Mn going substitutionally in the Bi sites, Mn going into a Se site, and Mn cluster formation. For the Mn in the Bi site model, Se1.1 and Se2.1 refer to the Se1 and Se2 nearest neighbors of a $\mathrm{Bi}$ atom, corresponding to the Se1 and Se2 sites shown in Fig. 4.7(c). The data and the magnitude of real part of $\chi(R)$ with the best fitting results are 
represented by the curves in Fig. 4.7 with parameters shown in 4.2 . We only considered the nearest Bi sites when analyzing the $\mathrm{Mn}$ in Se1 site. For the case of $\mathrm{Mn}$ in Bi sites (red curves), the data were fit very well by including scattering from the Se1 and Se2 sites, with an R-factor of 0.0031. Interestingly, the data indicate that the Se nearest neighbors are much closer than if the sites were occupied by Bi. The Mn in Se1 site fit (blue curve) was qualitatively different from the data, showing a dip near the first-shell maximum characteristic of a Ramsauer-Townsend resonance resulting from scattering from the heavy atoms (a similar result is obtained for substitution in the Se2 site) [81]. For the Mn cluster model, the results indicate an anomalously low scattering amplitude $\left(S_{0}^{2}=0.18\right)$. Moreover, although the shape of the main peak appears to be approximately correct, the structure around it is not reproduced by this model in terms of the positions of the secondary maxima and minima. This leads to an R-factor that is significantly higher than for the Mn in Bi site model (0.051). Therefore,our results support doping into the group V element site, in agreement with a previous EXAFS study of Mn-doped GaAs, [82] and disprove the Mn segregation scenario. Our results are in agreement with recent EXAFS work on $\mathrm{Cr}$ doped $\mathrm{Bi}_{2} \mathrm{Se}_{3}$ single crystals where $\mathrm{Cr}$ was found to substitute primarily into the Bi sites with a significant local contraction of the Cr-Se bond (2.50 $\AA$ ) [83]. The Mn-Se bond lengths obtained from EXAFS were shorter than those corresponding to Bi-Se bonds in undoped $\mathrm{Bi}_{2} \mathrm{Se}_{3}$ ( $\mathrm{R}$ and Re, respectively, listed in table 4.2). In order to determine whether the bond lengths corresponding to the nearest neighbor paths determined from EXAFS are sensible, first-principles density functional theory (DFT) calculations were performed using the Vienna Ab-initio Simulation Package (VASP) $[84,85]$, which uses pseudopotentials together with the projector augmented wave approach. Starting with undoped $\mathrm{Bi}_{2} \mathrm{Se}_{3}$ lattice parameters $a=4.138 \AA$ and 


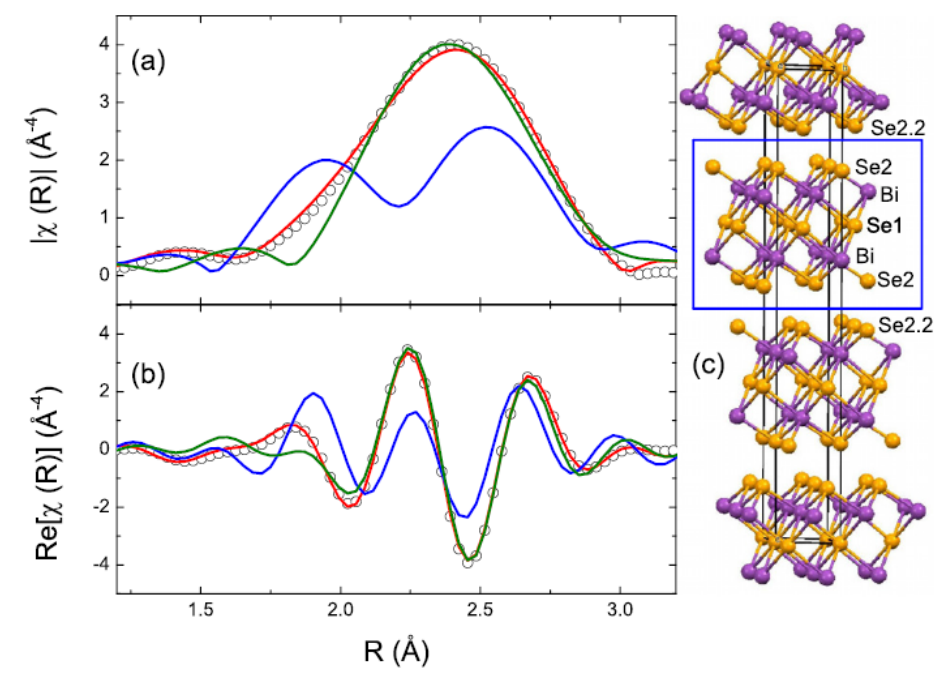

Figure 4.7: EXAFS Fourier transform function $\chi(R)$, of the response function $k^{3} \chi(k)$ near the Mn K $\alpha$ absorption edge for the $x=0.063$ sample in the range 1.2 Åto 3.2 $\AA$. The results of the fits to the magnitude (a) and real part (b) of $\chi(R)$ are shown the $\mathrm{Mn}$ atom in the $\mathrm{Bi}$ and Se1 sites (red and blue curves, respectively). The case of possible metallic Mn clustering corresponds to the green curve. The unit cell corresponding to three $\mathrm{Bi}_{2} \mathrm{Se}_{3}$ QLs is shown in (c). A single QL is outlined by the blue square and the $\mathrm{Bi}$ and Se atomic sites, composed of layers along the (vertical) c-axis are indicated. Note that the Se1 and Se2 sites are inequivalent. The Se2.2 sites are Se2 sites in adjacent QLs.

$c=28.64 \AA$, the unit cell was relaxed to obtain the minimum energy geometry. The forces on each of the atoms were calculated using the Hellmann-Feynman theorem, and were subsequently used to perform a conjugate gradient structural relaxation. The structural optimizations were continued until the forces on the atoms converged to less than $1 \mathrm{meV} / \AA$. This optimization was completely carried through for both undoped and doped materials. A sufficiently high-energy cutoff of $350 \mathrm{eV}$ was used in each calculation to obtain accurate results. The relaxed geometry of undoped $\mathrm{Bi}_{2} \mathrm{Se}_{3}$ yielded $a=4.184 \AA$ and $c=30.84 \AA$. 


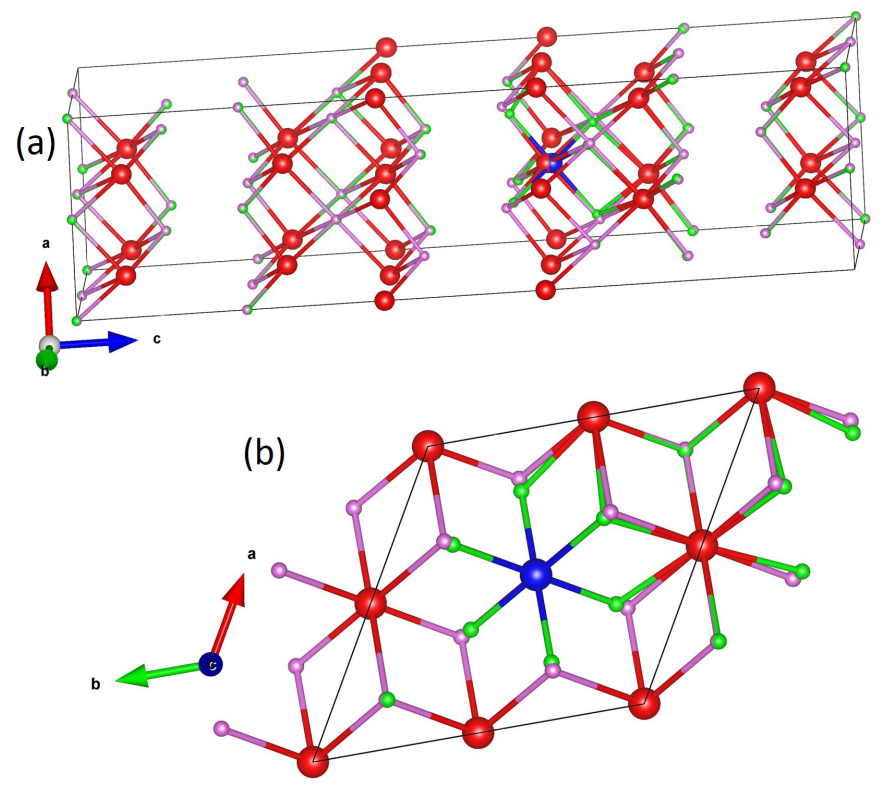

Figure 4.8: Unit cell used for the DFT calculations. Red spheres are Bi atoms, magenta spheres are Se atoms corresponding to pure $\mathrm{Bi}_{2} \mathrm{Se}_{3}$, green spheres are $\mathrm{Se}$ atoms corresponding to $\mathrm{Bi}_{2-x} \mathrm{Mn}_{x} \mathrm{Se}_{3}$, and the blue sphere is a $\mathrm{Mn}$ atom. The doped and undoped structures are superimposed to show the difference between them. (b) Perspective along the [001] direction (c-axis) of the quintuple layer containing the Mn impurity. Visualization rendered using the VESTA software package [86].

The calculated distance between first nearest Se-Bi neighbors was $2.88 \AA$ and $3.00 \AA$, which are within $3 \%$ of the experimental values determined for single crystals of $2.85 \AA$ and $3.07 \AA$, respectively [80]. The reason that the calculated lattice parameter disagreed significantly along the c-axis is the difficulty of the theory in calculating the Van der Waals interaction between adjacent Se-Se planes, but otherwise the bond length calculations were quite similar to our experimental observation. For the case of the Mn-doped system, we used a $2 \times 2 \times 1$ super-cell and replaced one out of $24 \mathrm{Bi}$ atoms by a Mn atom, as shown in Fig. 4.8a, corresponding to $x=0.084$. The lattice constants obtained for the Mn-doped structure were $a=4.173 \AA$ and 
4.2. GROWTH AND STRUCTURAL CHARACTERIZATION

\begin{tabular}{|cccccccc|}
\hline Site & R-factor & Path & $\mathrm{N}$ & $\sigma^{2}\left(\AA^{2}\right)$ & $S_{0}^{2}$ & $R(\AA)$ & $R_{e}(\AA)$ \\
\hline $\mathrm{Bi}$ & 0.0031 & $\mathrm{Se} 2.1$ & 3 & $0.013 \pm 0.007$ & $0.9 \pm 0.2$ & $2.66 \pm 0.03$ & 2.851 \\
& & $\mathrm{Se} 1.1$ & 3 & $0.013 \pm 0.006$ & $0.9 \pm 0.2$ & $2.78 \pm 0.03$ & 3.075 \\
Se1 & 0.53 & $\mathrm{Bi}$ & 6 & 0.025 & $0.1 \pm 0.2$ & $2.44 \pm 0.03$ & 3.074 \\
Mn Cluster & 0.051 & $\mathrm{Mn}$ & 12 & $0.008 \pm 0.003$ & $0.18 \pm 0.05$ & $2.80 \pm 0.02$ & 2.752 \\
\hline
\end{tabular}

Table 4.2: Results of fits to EXAFS data for $1.2 \AA<R<3.2 \AA$. For the Mn in Bi site, only nearest Se atoms at Se2 and Se1sites were considered, while for the Mn in Se1 site, only the nearest Bi sites were taken into account. The parameters $S_{0}^{2}$ and $R$ are the amplitudes and shell distances obtained from the fits, while $N$ and $R_{e}$ are the expected degeneracy for each shell (number of atoms) and bond distances for the $\mathrm{Bi}_{2} \mathrm{Se}_{3}$ structure, [80] respectively (not fitting parameters). For the Mn cluster model, the shortest bond distance for the a-Mn structure is listed. [89] The DebyeWaller scattering factor $\sigma^{2}$ was a fitting parameter for the fits using the $\mathrm{Mn}$ in $\mathrm{Bi}$ and Mn cluster models. The number of equivalent scatterers $\mathrm{N}$ was left as a fixed value during the fitting process. Uncertainties are as reported by Artemis.

$c=30.89 \AA$. Our calculations indicated that the Bi-Se bond lengths were $2.68 \AA$ and $2.75 \AA$, as shown in Fig. 4.8b, which agree well with the values of $2.66 \AA$ and $2.78 \AA$ determined from EXAFS data (Table 4.2). These results were also consistent with recent DFT calculations of the structure of Mn and other transition metal impurities near the surface of $\mathrm{Bi}_{2} \mathrm{Se}_{3}$, which found bond lengths of $2.60 \AA$ and $2.70 \AA$ for $\mathrm{Mn}$ in Bi sites just below the surface [87]. Those same calculations determined that the energies of other configurations, including interstitial sites for $\mathrm{Mn}$, are energetically unfavorable, and moreover, the calculated bond lengths from these other configurations were significantly different from the EXAFS values. Finally, the EXAFS results are also consistent with $\mathrm{Mn}$-Se bond lengths in $\mathrm{MnSe}$ and $\mathrm{MnSe}_{2}(2.71 \AA$ and 2.73 $\AA$, respectively) [88], thus providing additional evidence that, in our samples, the Mn atoms are predominantly incorporated substitutionally into the Bi sites.

In a similar study with $\mathrm{Cu}$ doped $\mathrm{Bi}_{2} \mathrm{Se}_{3}$ single crystals [90], it was shown that $\mathrm{Cu}$ can be either substituted into Bi sites or in the Van der Waals gap between two Se layers. Intercalation scenario can lead to superconductivity in these single 
crystals. Morevoer, it has been reported that $\mathrm{Cu}$ can also be absorbed into interstitial or intercalated sites in the $\mathrm{Bi}_{2} \mathrm{Se}_{3}$ lattice [91]. In the case of $\mathrm{Cr}$ doping, it has been claimed that the intercalation of $\mathrm{Cr}$ was consistent with the expansion of out of plane lattice parameter [64]. We also observed an increase in the $c$ lattice parameter which accompanied with a decrease in the in plane alattice constant as shown in figure $4.4 \mathrm{~b}, \mathrm{c}$. The volume of the unit cell was approximately constant with Mn concentration. Both XRD and EXAFS data implied the Mn substitutes for Bi and the increase in the out of plane lattice constant is not a result of intercalation scenario. We claim that the reason for the expansion of $c$ lattice parameter may be the weakening of Van der Waals interaction between the quintuple layers.

Our DFT calculations performed by Kartick Tarafder [92], gave the correct qualitative trend for the changes in lattice parameters. The experimental data for the sample $x=0.063$ showed an increase of $0.6 \%$ of $c$ lattice constant with a decrease of $0.7 \%$ for the $a$ lattice constant. On the other hand, DFT calculations yielded an expansion of $0.2 \%$ for out plane and $0.3 \%$ contraction for the in plane lattice parameters. The disagreement in numbers maybe due to the difficulty of calculating Van der Waals interaction by using ab-initio methods. We conclude that the the changes in lattice parameters and the EXAFS data with DFT calculations are consistent with Mn substitution for $\mathrm{Bi}$ in $\mathrm{Bi}_{2} \mathrm{Se}_{3}$ lattice. 


\subsection{Electrical Characterization}
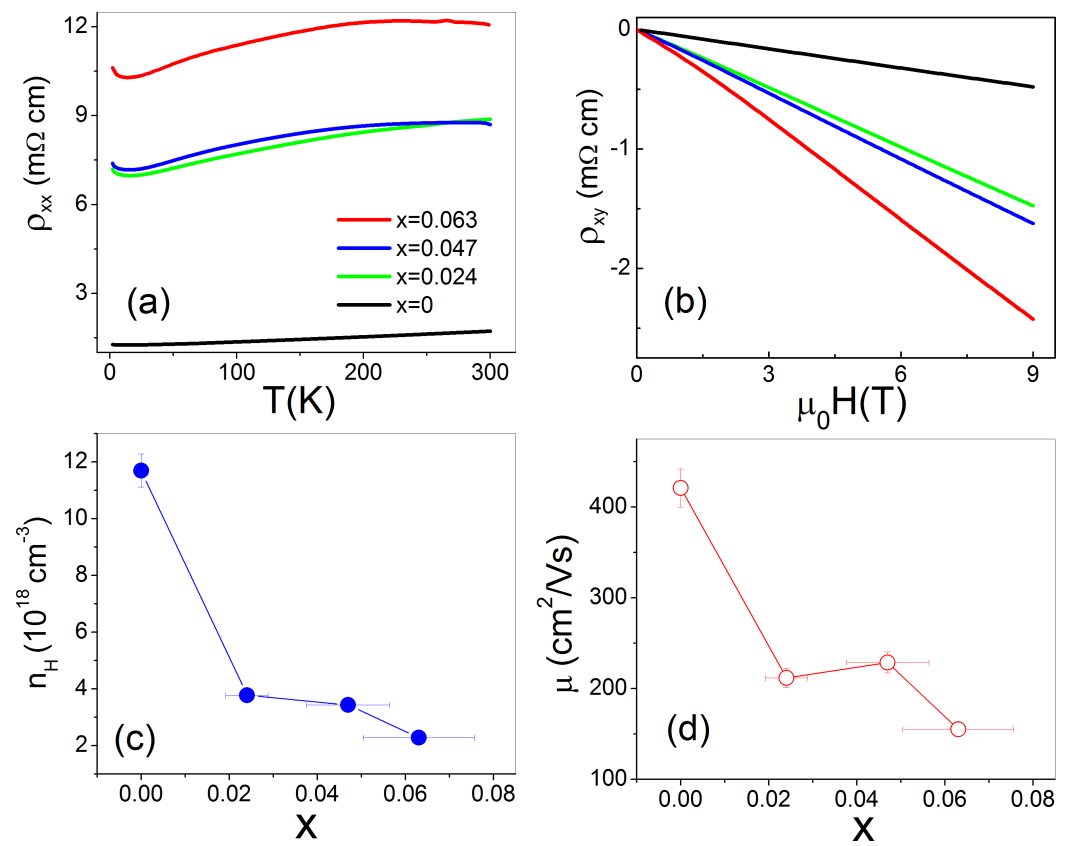

Figure 4.9: (a) Temperature dependence of longitudinal resistivities. (b) Hall resistivity data at $T=2 K$ for different $M n$ concentrations as a function of magnetic field. (c) Charge carrier density $n_{H}$ and (d) mobility $\mu$ as functions of Mn-doping concentration.

The longitudinal film resistivity $\rho_{x x}$ was measured as a function of temperature in zero field as shown in Fig. 4.9(a). The resistivity of the film with $x=0.063$ was approximately an order of magnitude larger than the resistivity of the undoped sample, as expected from the increased scattering from magnetic Mn impurities and a larger density of structural defects. Temperature dependences had a strong metallic character for all samples at high temperatures. At temperatures below $15 \mathrm{~K}$, however, the resistivity of all samples increased as the temperature was lowered. Similar findings have been reported [93] for $\mathrm{Bi}_{2} \mathrm{Se}_{3}$ films with thickness $\mathrm{t} \leq 10 \mathrm{QL}$, which 
have been explained by the Altshuler-Aronov-Lee (AAL) mechanism [94] where weak disorder results in a non-local effective enhanced electron-electron interaction. Carrier densities, $n_{H}$, were calculated from the linear slopes of the corresponding Hall resistivities measured at $T=2 \mathrm{~K}$ which are shown in Fig. 4.9b. All samples had n-type carriers and the bulk charge carrier density decreased with increasing $\mathrm{Mn}$ concentration, as shown in Fig. 4.9c. This indicates that the Mn impurities acted as acceptors that compensated the bulk carriers. This compensation could occur if the valence of $\mathrm{Mn}$ was $2+$ instead of $3+$, which is consistent with the structural data discussed above. The mobilities were calculated using the standard equation $\mu=\left(\mathrm{e} \rho_{x x} n_{H}\right)^{-1}$, where $e$ is the charge of the electron. The decrease in mobility with increasing Mn-doping, shown in Fig.4.9d, is due to the increased electron scattering from defects. It is important to note that the decrease in the number of carriers with increasing Mn concentration is modest (a factor of three) and much lower than expected if all of the Mn sites acted as acceptors. However, the structural data indicate that incorporation of Mn also adds structural defects, possibly grain boundaries and/or Se vacancies, for example. These structural defects could have the opposite effect, adding carriers to the conduction band at the same time that the Mn sites act as acceptors. Alternatively, increasing Mn concentration can change the width of charge depletion layer in the bulk and may result in a non-linear decrease in carrier density. The detailed discussion for the changes observed in the carrier density in pure and Mn doped samples will be presented in the next chapter.

\subsection{Conclusions}

$\mathrm{Bi}_{2-x} \mathrm{Mn}_{x} \mathrm{Se}_{3}$ thin films were grown using molecular beam epitaxy and their structure was systematically investigated using x-ray diffraction, x-ray reflectivity, XPS, 
and EXAFS. The structural quality of the samples was maintained for 30 QL thick samples with $x \leq 0.063$. It was verified that the Mn atoms were incorporated substitutionally into the Bi sites with a $\mathrm{Mn}^{2+}$ valence using XPS, EXAFS, and DFT calculations. No evidence of intercalated incorporation of $\mathrm{Mn}$ was found, unlike $\mathrm{Cu}$ doped $\mathrm{Bi}_{2} \mathrm{Se}_{3}$, where both intercalation and substitutional incorporations have been identified. The behavior of the electrical resistivity and mobility as a function of temperature was consistent with Mn impurities acting as acceptors for Mn substitution in Bi sites. Structural defects created by Mn incorporation result in additional donors that mitigate the compensation effects due to $\mathrm{Mn}^{2+}$ acceptors. 


\section{Chapter 5}

\section{Single vs. Three Channel HLN}

\section{Model for $\mathrm{Bi}_{2-x} \mathrm{Mn}_{x} \mathrm{Se}_{3}$ Thin Films}

\subsection{Introduction}

The electrons residing on the surface of a TI theoretically should have nearly dissipation-less transport despite the presence of non magnetic impurities. Due to the metallic surface states, TIs are accepted as potential candidates for room temperature spintronics and quantum computing applications. Therefore, it is crucial to study the electronic behavior and the transport properties in topological insulators. The interpretation of the magnetotransport measurements on TIs is rather challenging due to the intrinsic defects that form during the growth of the material. These defects have donor characteristics and results in conducting bulk states. The main challenge in experiments is to separate the conductivity of the bulk and surface channels. There are several different approaches to this problem; doping with an acceptor to compensate the donor type defects $[68,95]$, mixing the different layers of $\mathrm{Sb}$ and $\mathrm{Te}$ in $\left(\mathrm{Bi}_{x} \mathrm{Sb}_{1-x}\right)_{2} \mathrm{Te}_{3}[96]$ and $\left(\mathrm{Bi}_{1-x} \mathrm{Sb}_{x}\right)_{2} \mathrm{Te}_{3}$ [97], and decreasing the film thickness to 
maximize the surface to volume ratio. MBE growth yields a high crystalline quality and fine control of quintuple layer thickness in the case of TI thin films and nano structures.

Here the electrical transport properties for a series of pure $\mathrm{Bi}_{2} \mathrm{Se}_{3}$ and Mn doped $\mathrm{Bi}_{2-x} \mathrm{Mn}_{x} \mathrm{Se}_{3}$ thin films grown by MBE are discussed. The $\mathrm{Bi}_{2} \mathrm{Se}_{3}$ film thickness was kept in 6 - 50 QL range and the $\mathrm{Bi}_{2-x} \mathrm{Mn}_{x} \mathrm{Se}_{3}$ layer thickness was fixed to $12 \mathrm{QL}$ with $\mathrm{Mn}$ doping ratio, $x$ was varied in range $0 \leq x \leq 0.2$. Hall effect measurements indicated a decrease in carrier density and an increase in mobility as a function of increasing film thickness for pure samples. Hall effect measurements of the Mn doped samples indicated an increase in the carrier density with Mn doping and a large decrease in mobility due to the scattering from magnetic impurities. It is important to note that the change in the carrier density in Mn doped samples depended on the relative competition between $\mathrm{Mn}^{2+}$ acceptors and the changes in the density of donor defects such as Se vacancies (double donor), Se antisites $\left(\mathrm{Se}_{B i}\right)$ and $\mathrm{Bi}$ antisites $\left(\mathrm{Bi}_{S e}\right)$ forming during the $\mathrm{Mn}$ addition [98]. The low temperature behavior of resistance indicated that the logarithmic increase is due to enhanced electron electron interaction (EEI) in the pure samples and a combination of EEI and Kondo anomaly in Mn doped samples. 2D magnetoconductance was studied in perpendicular magnetic field by using simplified HLN model with two fitting parameters; $\alpha$ and phase coherence length $\left(\mathrm{L}_{\phi}\right)$. The fitting parameter $\alpha \approx-0.5$ interpreted as a single channel in all of the pure and lowly-doped samples. In samples with high Mn concentration $\alpha \rightarrow 0$, as expected when magnetic scattering is strong. The generally accepted version of this simplified HLN model does not distinguish the surface from bulk channels independently. The fitting parameter $\alpha$ is used to estimate the number of independent channels in the samples. On the other hand, the concept of phase 

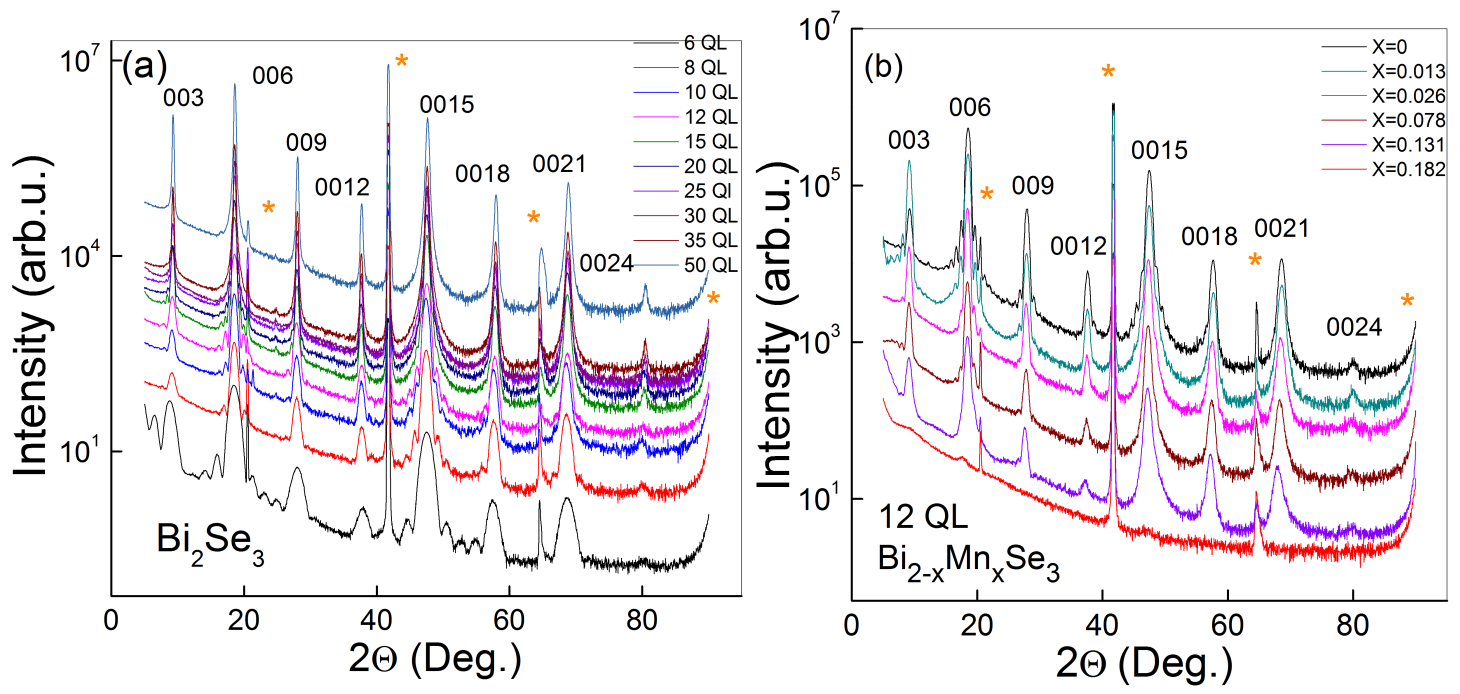

Figure 5.1: (a) X-ray diffraction data for the pure samples in range 6-50 QL. The (003) family of peaks is indicated in the figure. Asterisks represent the reflections from the $\mathrm{Al}_{2} \mathrm{O}_{3}$ substrate. (b) X-ray diffraction spectra for $12 \mathrm{QL} \mathrm{Bi}_{2-x} \mathrm{Mn}_{x} \mathrm{Se}_{3}$ samples. All the films are oriented in c-axis, out of plane with absence of secondary phases. The absence of Bragg peaks in the sample with $x=0.182$ indicates the amorphous nature of this film.

coherence length is more ambiguous than the parameter $\alpha$. It does not give any information on relative phase coherence lengths for the surface and bulk channels, even for the case where two channel dominates transport $(\alpha=1)$. In order to address all of these concerns, we will discuss the HLN model with a single vs. three channel formulation of perpendicular magnetoconductance.

\subsection{Growth and Structural Characterization}

Thin films of $\mathrm{Bi}_{2} \mathrm{Se}_{3}$ with a thickness of $6-50 \mathrm{QL}$ and $\mathrm{Bi}_{2-x} \mathrm{Mn}_{x} \mathrm{Se}_{3}$ with a thickness of 12 QL with $x=0,0.013,0.026,0.078,0.131,0.182$ were grown via MBE as described in Chapter 4. The surface of the films was monitored in-situ with RHEED. The sample thickness and the Mn concentration were calibrated by a quartz crys- 

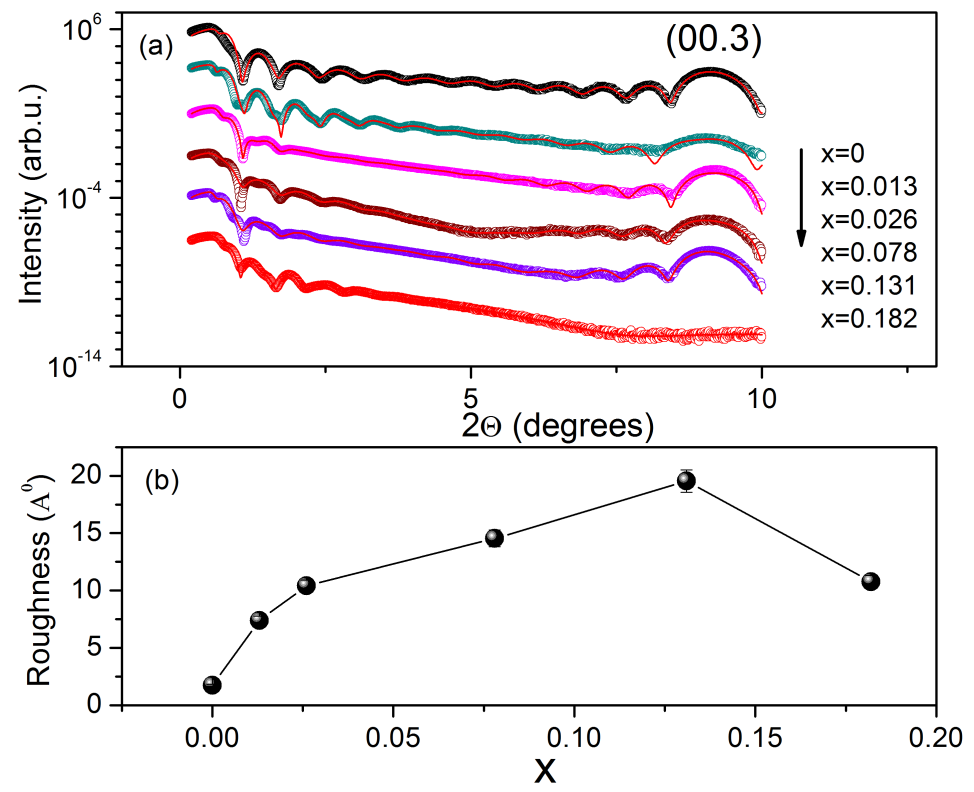

Figure 5.2: (a) X-ray reflectivity data for the $12 \mathrm{QL} \mathrm{Bi}_{2-x} \mathrm{Mn}_{x} \mathrm{Se}_{3}$ thin films. The solid red lines are the fits to the data. (b) Roughness as a function of $\mathrm{x}$ in $\mathrm{Bi}_{2-x} \mathrm{Mn}_{x} \mathrm{Se}_{3}$ thin films.

tal microbalance (QCM). X-ray diffraction (XRD) measurements indicated that all of the films were single phase and oriented out of plane along the [001] hexagonal direction as shown in Fig. 5.1.

X-ray reflectivity (XRR) data and the corresponding fits were used to confirm the thicknesses with an uncertainty of $5 \%$. The roughness values obtained via fitting XRR data indicated a roughness of less than 1 QL in pure samples. Figure 5.2 represents the XRR data and the corresponding fits for the 12 QL Mn doped samples as a function of Mn doping concentration. In order to detect the first Bragg peak (003), the scan range was restricted to $0.2^{\circ} \leq 2 \theta \leq 10^{\circ}$. The increasing disorder resulted in an increase in the RMS roughness values as shown in Fig. 5.2b. The highly doped sample with $x=0.182$ did not show the (003) Bragg peak similar to the high angle XRD measurements and is therefore probably amorphous (Fig. 5.1b). 

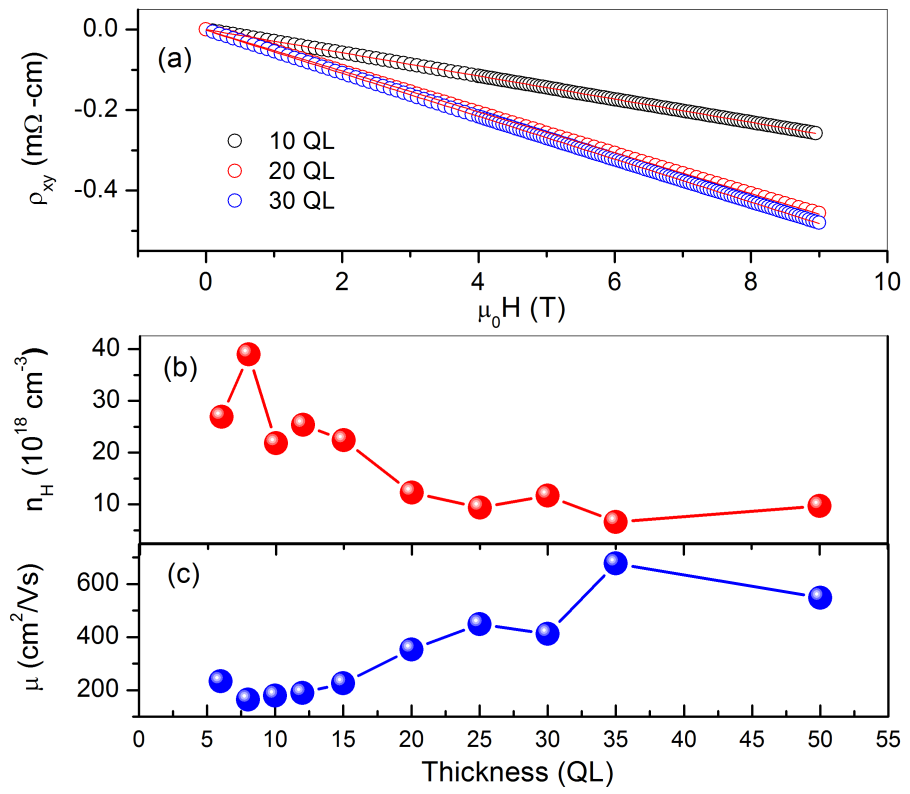

Figure 5.3: (a) Hall resistivity vs. magnetic field for three representative samples with a thickness of 10, 20 and 30 QLs. Red lines represent the linear fits to the data. (b) Volume carrier density, $\mathrm{n}_{H}$ vs. thickness in QL. (c) Mobility, $\mu$ vs. $\mathrm{Bi}_{2} \mathrm{Se}_{3}$ slab thickness.

\subsection{Carrier Density and Hall Mobility}

The Hall effect measurements were performed at $T=2 \mathrm{~K}$ in a magnetic field of 9 Tesla, which was applied perpendicular to the film surface. Bulk carrier densities were extracted from the linear slopes of Hall resistivity data. Figure 5.3a shows a typical Hall measurement data for three representative samples with thickness of 10, 20 and 30 QLs. Satisfactory linear fits to the Hall resistivity were obtained which indicated that major charge carriers were electrons. The same analysis was done for all of the other samples which yielded linear Hall resistivity similar to the data shown in Fig. 5.3a. The mobility were calculated using the equation $\mu=\left(\mathrm{e} \rho_{x x} n_{H}\right)^{-1}$. The carrier density (Fig. 5.3b) and mobility (Fig. 5.3c) as a function of pure $\mathrm{Bi}_{2} \mathrm{Se}_{3}$ slab thickness were calculated from these measurements. In an ideal topological insulator the bulk is insulating, but in experiments this is extremely difficult to achieve due 
to native defects in $\mathrm{Bi}_{2} \mathrm{Se}_{3}$. If the bulk of the film is insulating, then there should be no change in the mobility and the carrier density as a function of thickness since the transport would be entirely due to the surface states. This situation would be different for thin films with a thickness $\mathrm{t} \leq 6 \mathrm{QL}$ where quantum tunneling between top and bottom TI surface states destroys the topological protection. For most of our $\mathrm{Bi}_{2} \mathrm{Se}_{3}$ films, this was not a concern since the thickness was larger than 6 QL. The increase in mobility and a decrease in carrier density as a function of thickness implies that the transport was increasingly dominated by the bulk states with increasing thickness [99]. Bulk carrier density in $\mathrm{Bi}_{2} \mathrm{Se}_{3}$ mainly originates from the donor type defects such as selenium vacancies $\left(\mathrm{V}_{S e}\right)$, Se antisite $\left(\mathrm{Se}_{B i}\right)$, and $\mathrm{Bi}$ antisite $\left(\mathrm{Bi}_{S e}\right)$ defects. Se vacancies are thermodynamically the most stable defects in $\mathrm{Bi}_{2} \mathrm{Se}_{3}$ crystal structure and the number of Se vacancies may increase with the decreasing film thickness [99]. Also, the diffusion of these donors over time may change the value of free carrier density in the material. The decreasing carrier density with increasing film thickness may indicate that the donor type defects are more in the thinner films [99]. However, our RHEED, XRD and XRR data shows that the number of defects and disorder level decreases in thinner films for our samples [100], which is contradictory to the increasing carrier density in thinner films, if defects were considered as the only mechanism for this behavior. Therefore, the increase in the carrier density with decreasing $\mathrm{Bi}_{2} \mathrm{Se}_{3}$ film thickness can not be explained by Se vacancies or donor defects (Fig. 5.3b). The increase in carrier density in the thinnest films results from the fact that the transport is more surface sensitive as film thickness is reduced and the high density of surface states becomes more important.

The 12 QL Mn doped $\mathrm{Bi}_{2-x} \mathrm{Mn}_{x} \mathrm{Se}_{3}$ films showed a nonmonotonic behavior in carrier density with increasing $x$ and extremely low mobilities in highly doped samples 
(Fig. 5.4c and Fig. 5.4d). The temperature dependence of resistivity was measured in the range of $2 \mathrm{~K} \leq T \leq 300 \mathrm{~K}$ and revealed metallic behavior at high temperatures as shown in Fig. 5.4a. Similar high temperature behavior in resistivity was observed in all of our thin films, including pure $\mathrm{Bi}_{2} \mathrm{Se}_{3}$, which can be attributed to the large number of native defects in $\mathrm{Bi}_{2} \mathrm{Se}_{3}$. The absolute value of resistivity increased with increasing Mn concentration due to increased scattering from magnetic impurities and enhanced disorder.
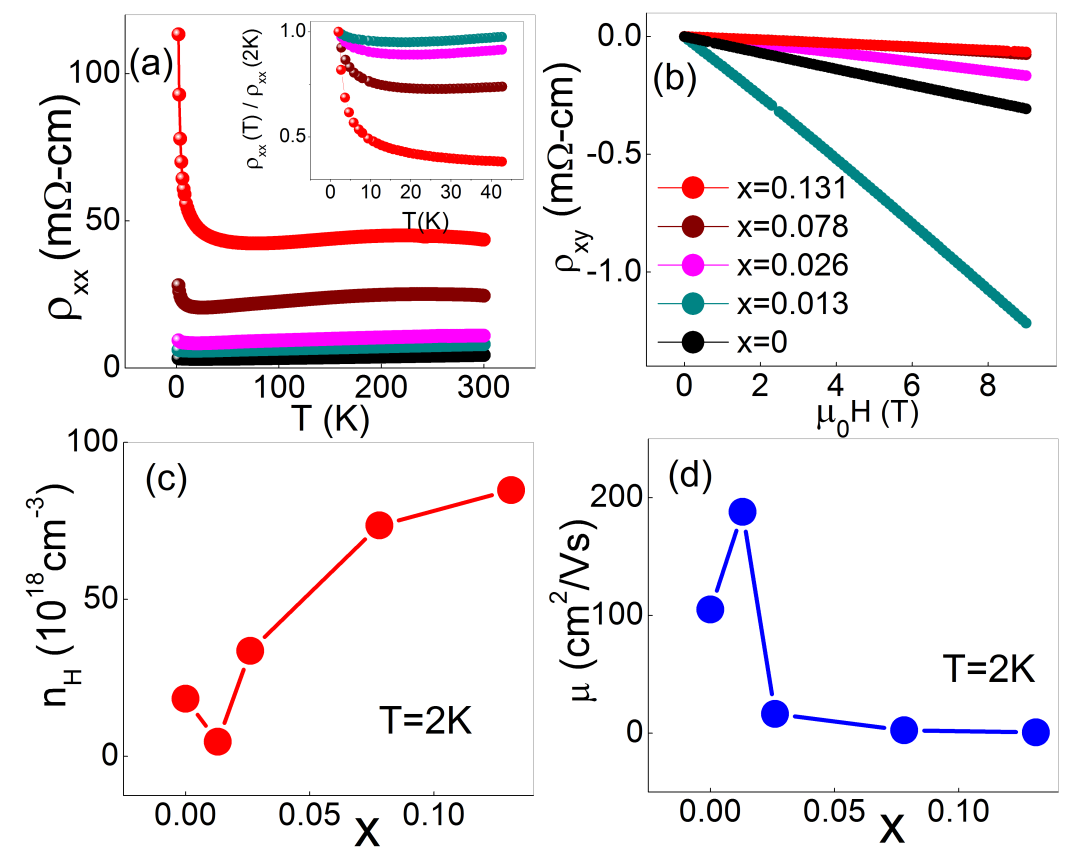

Figure 5.4: a) Resistivity vs. Temperature for different $x$ in $\mathrm{Bi}_{2-x} \mathrm{Mn}_{x} \mathrm{Se}_{3}$ (b) Hall resistivity as a function of magnetic field at $T=2 \mathrm{~K}$ (c) 3D-bulk charge carrier density, $n_{H}$ vs. $x$ (d) Mobility $\mu$ as a function of $x$ in $\mathrm{Bi}_{2-x} \mathrm{Mn}_{x} \mathrm{Se}_{3}$.

Another important finding from the resistivity measurements is a partial Kondo contribution with increasing Mn concentration. The temperature at which the resistivity reaches a minimum shifts to higher temperatures with Mn impurity level (Inset to Fig. 5.4a). This type of behavior in resistivity minimum is expected from 
the Kondo anomaly. As a result of the Kondo effect, at sufficiently low temperatures, conduction electrons are screened by magnetic impurities and results in a logarithmic contribution to the resistivity [101].

The Hall effect was measured in the same way as reported for the pure films (Fig. 5.3a). The Hall effect data for Mn doped $12 \mathrm{QL} \mathrm{Bi}_{2-x} \mathrm{Mn}_{x} \mathrm{Se}_{3}$ is shown in Fig. 5.4b. N-type conductivity with the electron carriers dominated the transport (Fig. 5.4b). A nonmonotonic behavior as a function of $x$ was observed in carrier density (Fig. 5.4c) and mobility (Fig. 5.4d) as shown above. The nonmonotonic feature in carrier density and mobility can be understood by the formation of depletion layer which occurs as a result of the changes in charge redistribution with film thickness. As the film thickness is reduced, the band structure is distorted due to space-charge accumulation and a charge depletion layer is formed with a finite width, $z_{d}[102]$. The width of this depletion layer can be estimated via Poisson equation, $z_{d}^{2}=\kappa \epsilon_{0} \Delta V / e n_{H}$, where $\kappa$ is the dielectric constant of the material, $\Delta V$ is the energy difference between surface and bulk states, and $n_{H}$ is the carrier density. It is clear that the depletion width, $z_{d}$, is inversely proportional to the carrier density, $n_{H}$. The critical thickness where the sum of two depletion widths (from top and bottom surfaces) become comparable to the film thickness occurs around $12 \mathrm{QL}$ with a typical carrier density of $2.5 \times 10^{19} \mathrm{~cm}^{-3}$ [103]. Quantitatively, for a $12 \mathrm{QL} \mathrm{Bi}_{2} \mathrm{Se}_{3}$ film, depletion width is $6.5 \mathrm{~nm}$ for each surface, making a total of $13 \mathrm{~nm}$ for the whole film. On the other hand, Mn doping causes a partial carrier compensation in $\mathrm{Bi}_{2-x} \mathrm{Mn}_{x} \mathrm{Se}_{3}$ thin films, indicating that the depletion width increases in doped samples. Increasing Mn concentration causes an enhancement in the depletion width and redistributes charges towards the surfaces. As a result of charge redistribution towards surfaces due to Coulomb repulsion, carrier density increases in $12 \mathrm{QL}$ Mn doped films. On the 
other hand, for $30 \mathrm{QL}$ thick $\mathrm{Bi}_{2-x} \mathrm{Mn}_{x} \mathrm{Se}_{3}$ thin films, the carrier density decreases as a function of $x$ (see Fig. 4.9c in chapter 4). It must be recalled that $\mathrm{Mn}^{2+}$ is acceptor and causes an increase in the depletion width regardless of the film thickness. Therefore, for $30 \mathrm{QL} \mathrm{Bi}_{2-x} \mathrm{Mn}_{x} \mathrm{Se}_{3}$ thin films, the depletion width is also enhanced by Mn but the total depletion width is probably smaller than the film thickness and the contribution from surface states is not pronounced. This results in an overall decrease in carrier density for thicker films which saturates when Mn concentration reaches, $x=0.064$ in $30 \mathrm{QL} \mathrm{Bi}_{2-x} \mathrm{Mn}_{x} \mathrm{Se}_{3}$. This picture is consistent with the general interpretation of Hall carrier density, $n_{H}$, where $n_{H}=n_{b}(1-c x)+n_{s}(1+b x) / d$ is the total carrier density measured in a Hall experiment. In above formula, $d$ is the film thickness, $x$ is the Mn concentration, $n_{b}$, and $n_{s}$ are the volume and sheet carrier densities, and $b$ and $c$ are arbitrary constants. The charge migration towards surfaces decreases the value of the bulk carrier density, while it increases the sheet carrier density. It must be mentioned that the second term, $n_{s}(1+b x) / d$, in above formula becomes dominant as the film thickness is reduced. Therefore, the effect of Mn on carrier density depends on the charge migration towards the surfaces and the depletion width which determines the value of bulk carrier density $\left(n_{b}\right)$ and sheet carrier density $\left(n_{s}\right)$ in $\mathrm{Bi}_{2-x} \mathrm{Mn}_{x} \mathrm{Se}_{3}$ samples. Moreover, the mobility decreases due to increased scattering from defects and because of an increase in the number of magnetic impurities in the depletion layer or near the surface, which tends to kill the weak antilocalization. In order to have a complete understanding of carrier dynamics in Mn doped samples, more measurements need to be done in the future. 


\subsection{Single Channel HLN Fit Results for 12 QL $\mathbf{B i}_{2-x} \mathbf{M n}_{x} \mathbf{S e}_{3}$ Thin Films}

\subsection{1 $\alpha$ and Number of Channels}

Low field magnetoconductance was studied at low fields with two fitting parameters, $\alpha$ and $L_{\phi}$. In the low field regime, the correction to the magnetoconductance $\Delta \sigma(B)=\sigma(B)-\sigma(0)$ was fitted with the Hikami-Larkin-Nagaoka (HLN) formula $\Delta \sigma_{\perp}(B)=\alpha \frac{e^{2}}{2 \pi^{2} \hbar}\left[\psi\left(\frac{\hbar}{4 e L_{\phi}^{2} B}+\frac{1}{2}\right)-\ln \left(\frac{\hbar}{4 e L_{\phi}^{2} B}\right)\right]$, where $\alpha$ is a preconstant, $B_{\phi}$ is the dephasing field related to phase coherence length with $L_{\phi}$, via $B_{\phi}=\hbar /\left(4 e L_{\phi}^{2}\right)$, and finally $\psi$ is the digamma function. Since the 2D surface of a $3 \mathrm{D}$ TI belongs to a symplectic class, the expected values for $\alpha$ are; $\alpha=-0.5$ for one and $\alpha=-1$ for two decoupled channels. However, surface and the bulk states can be regarded as independent conducting channels as long as electrons keep their coherence before scattering in and out of another channel. In this case, WAL will be dominated by the time reversed loops of all channels independently and each channel will bring a -0.5 contribution to the prefactor $\alpha$. Therefore, the additive nature of preconstant $\alpha$ directly depends on the competition between the surface-to-bulk coupling $\left(\tau_{s b}\right)$ and the phase coherence time $\left(\tau_{\phi}\right)$. If $\tau_{s b}>\tau_{\phi}$, then each channel will carry a Berry phase of $\pi$ and contribute to WAL with a prefactor of $\alpha=-0.5$. On the other hand, if $\tau_{\phi}>\tau_{s b}$ charge carriers scatter between surface and bulk states and form an effective single channel.

There are several different interpretations for $\alpha=-0.5$. The first possibility is that the contribution is mainly coming from the top surface of TI, and not from the bulk state or from the bottom surface. A second possibility is that contribution from the surface states is negligible and the bulk acts as a $2 \mathrm{D}$ metallic channel. A 
third possibility is that the surface states contribute and couple to the metallic bulk states, and therefore the entire sample acts as a single $2 \mathrm{D}$ conducting channel. The first scenario assumes an insulating bulk and negligible conductivity from the bottom surface. A negligible bottom surface contribution could be explained by the lattice mismatch between the film and substrate which may cause $L_{\phi}$ significantly reduced at the bottom surface [104]. However, this scenario is not consistent with the robustness of TI surface states. Also, the assumption of a completely insulating bulk states which is not realistic, considering the fact that the TIs have high level of intrinsic defects that causes the Fermi level positioning well above the bulk conduction band minimum. In the second possibility, the bulk is treated as completely metallic with no contribution from the surfaces. In this case, the mobility and phase coherence length must scale linearly with increasing thickness if the growth parameters for all samples are the same. Our data do not support this hypothesis. The last and most accepted interpretation is that there is a coherently coupled surface to bulk single effective channel responsible for the WAL behavior of magnetoconductance. In this case, due to the presence of extended bulk states at or near the Fermi level, the surface electrons scatter in and out of bulk states. As a result, all conducting channels are intertwined and act as a single channel. However, this widely accepted interpretation fails to explain the deviations in the values of $\alpha$, which have been reported in the range of $0.38<-\alpha<1$, and it does not give any information on the nature of phase coherence length. In the next section we discuss how three independent channels can explain the data. 


\subsubsection{Phase Coherence Length and $\alpha$ Coefficient}

We carried out detailed analysis of the transport properties of 12 QL Mn doped $\mathrm{Bi}_{2} \mathrm{Se}_{3}$ thin films. Figure 5.5 demonstrates the HLN formula fits to the low field magnetoconductance with the corresponding fit parameters. The correction to the magnetoconductance is plotted in Fig. 5.5a. In the Mn doped samples, the shape of magnetoconductance is different from what is observed for the pure $\mathrm{Bi}_{2} \mathrm{Se}_{3}$ film (Fig. 5.5a). Changes in the shape of magnetoconductance in Mn doped samples result from the scattering of magnetic impurities. As $x$ increases, there is an extra phase contribution to the phase of the electron wave functions and the Berry phase is not equal to $\pi$ anymore. It is very interesting to mention that our lowly-doped sample also brings a prefactor $\alpha \approx-0.62$ as demonstrated in the Fig. 5.5(b). This value is almost identical to the undoped sample. Our observation naturally brings the question of whether the robustness of Dirac surface states might still be realized in the doped samples with very few magnetic impurities. In this respect, the evolution of $\alpha$ with $x$ suggests three separate regions to analyze. The pure sample showed a partial coupling to a second channel by $\alpha=-0.63$, which was also observed in lowly-doped sample with $\alpha=-0.62$. This is the region where the strong spin orbit scattering dominates the transport (Fig. 5.5b). The second region is the intermediate doping regime where $x=0.026$ and $\alpha=-0.84$, indicating a strong decoupling of two surfaces. This is consistent with another magnetic impurity doped system, Cu-doped $\mathrm{Bi}_{2} \mathrm{Se}_{3}$, where a very small $\mathrm{Cu}$ doping yields the same result with the undoped sample. Similar to our data, decoupling of two surfaces was also observed in the intermediate doping region for the case of $\mathrm{Cu}$-doped $\mathrm{Bi}_{2} \mathrm{Se}_{3}$ films [91]. The last region is where the magnetic scattering dominated over the spin orbit scattering and resulted in a change in the shape of magnetoconductance (Fig. 5.5a). In this region, HLN formula fits 

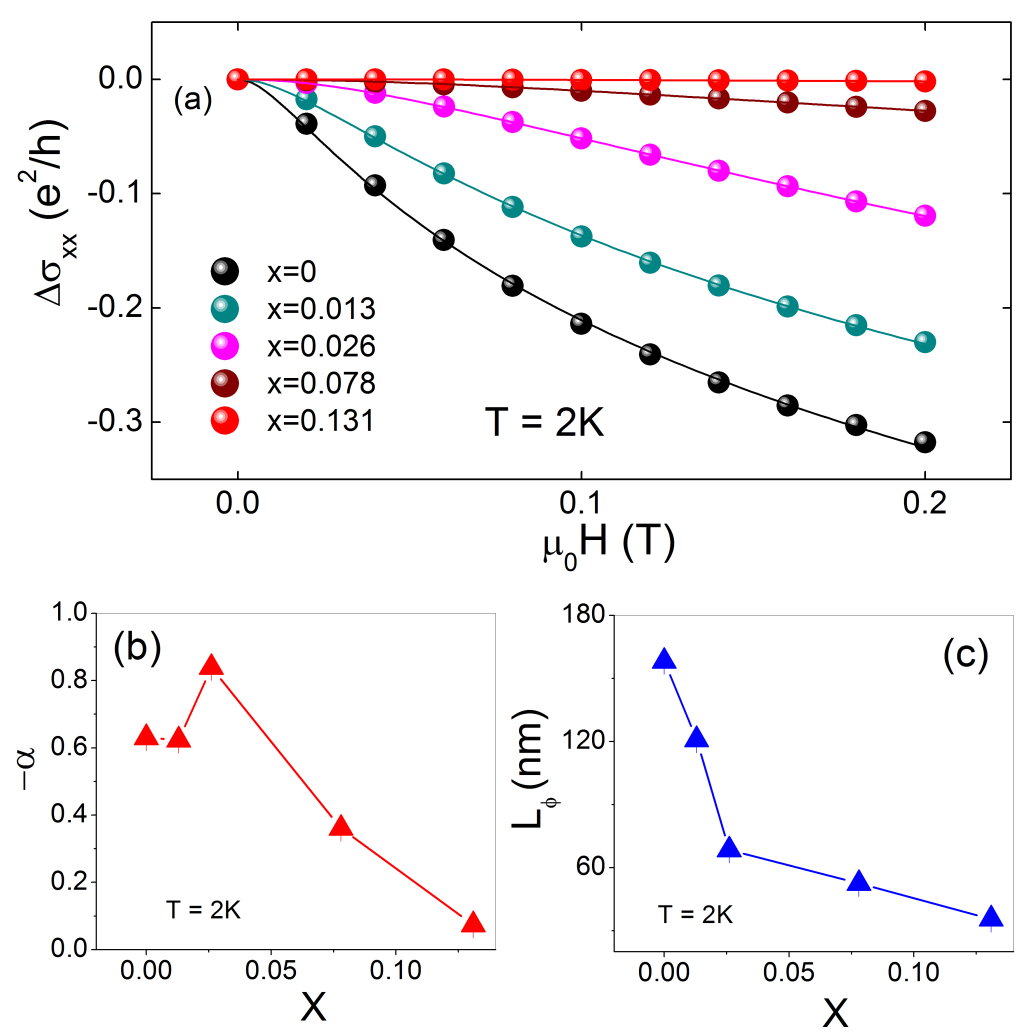

Figure 5.5: (a) HLN formula fits to magnetoconductance at low fields for Mn doped $\mathrm{Bi}_{2-x} \mathrm{Mn}_{x} \mathrm{Se}_{3}$ films with different $x$ values. Solid lines represent the fits to the 2D magnetoconductance at low fields. (b) The coefficient $\alpha$ vs. $x$. Three different regimes in the data are: single channel WAL with $\alpha \approx-0.5$ in pure and low-doped sample, a slow crossover to two decoupled channels in the intermediate doping region and finally a region where magnetic scattering dominates as $x$ increases significantly which results in $\alpha \approx 0.0$ (c) Phase coherence length $L_{\phi}$ vs. $x$, shows a large decrease in $L_{\phi}$ with increasing Mn concentration. The values are extracted from HLN formula fits to the magnetoconductance at $T=2 \mathrm{~K}$.

gave $\alpha \approx 0$ (Fig. 5.5b). In addition, our magnetoconductance measurements did not show a clear transition between WAL - WL characterized in the sign of $\alpha$. In such a case, a change in the shape of magnetoconductance is observed in low magnetic fields and preconstant $\alpha$ flips sign. Despite a breakdown of WAL was observed in highly-doped samples, there was no transition to WL, possibly due to the high quality of our films. The crossover between WAL - WL effect is characterized by 
the the competition of two time scales; short range inter-valley scattering $\left(\tau_{i v}\right)$ and phase coherence times $\left(\tau_{\phi}\right)$ in graphene [105]. The same effect is attributed to the competition between phase coherence $\left(\tau_{\phi}\right)$ and spin flip scattering times $\left(\tau_{s o}\right)$ in ultra thin films of $\mathrm{Bi}_{2} \mathrm{Se}_{3}$ [106]. In magnetically doped TIs, the transition WAL - WL, and negative magnetoresistance can be attributed to the relative ratio of TRS breaking gap $(\Delta)$ to the Fermi energy $\left(E_{f}\right)[23]$. The absence of WAL - WL transition in our samples suggests a small value for the ratio, $\Delta / E_{f}$ for $\mathrm{Mn}$ doped $\mathrm{Bi}_{2-x} \mathrm{Mn}_{x} \mathrm{Se}_{3}$ samples.

\subsubsection{Temperature Dependence of $\alpha$ and Channel Separa- tion}

The temperature dependence of magnetoconductance includes valuable information about the scattering mechanism in topological insulators. The shape of the curves is defined by the scattering mechanism which is related to the coherence length and to the number of conducting channels in the system. For each surface band, where momentum is locked to the spin degree of freedom, all time reversed paths around a closed trajectory acquire a $\pi$-Berry phase and brings a $\alpha=-0.5$ prefactor. In addition to the surface states, the bulk also shows WAL as it is a weakly disordered quasi-2D channel. As a result, both surface and the bulk states can contribute to the prefactor $\alpha$. The values of prefactor $\alpha$ in the range of $-0.39<\alpha<-1.1$ were reported in literature. These values suggest that one or two channels contribute to the WAL behavior even though some bulk carrier channels may co-exist with the surface channels. By fitting the temperature dependence of magnetoconductance curves with HLN formula, we systematically investigated the relation between WAL effect and the scattering mechanism in TIs. The values for $\alpha$ at $T=2 K$ were dis- 


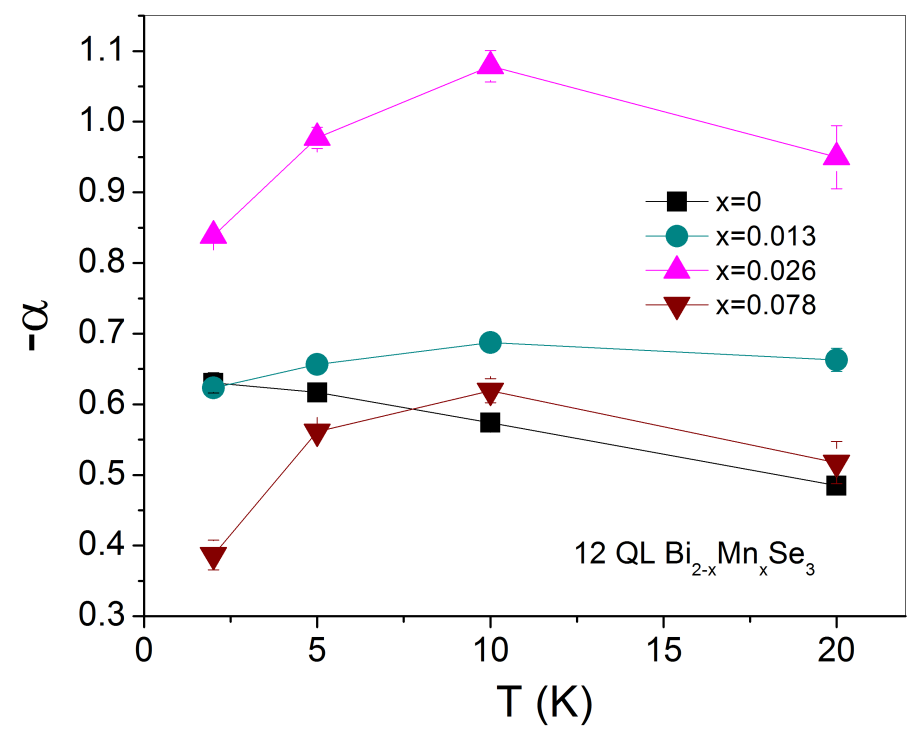

Figure 5.6: Temperature evolution of $\alpha$ for different $x$ in $\mathrm{Bi}_{2-x} \mathrm{Mn}_{x} \mathrm{Se}_{3}$ films. The channels are partially separated from each other with increasing Mn impurity level. Note that highly-doped sample, $x=0.131$ does not show any WAL at high temperatures and therefore is not included in this figure.

cussed previously (Fig. 5.5b) in three separate regions for different $x$ values. The temperature dependence of $\alpha$ were extracted for Mn doped samples which are shown in Fig. 5.6. The data clearly shows an increasing trend in the channel separation with increasing $\mathrm{Mn}$ concentration in the temperature range of $T \leq 10 \mathrm{~K}$ as shown in Fig. 5.6. The presence of Mn impurities lowers the scattering rates of charge carriers from surface to bulk channel and increases the ratio $\tau_{s b} / \tau_{\phi}$ (Fig. 5.6). As a result, an overall increase in $\alpha$ was observed for all of the Mn doped samples in the temperature range of $T \leq 10 K$. At $T>10 \mathrm{~K}$, electron-phonon scattering caused a channel mixing and decreased the value of $\alpha$ for Mn doped films. For pure film, increasing electron electron interaction (EEI) with increasing temperature caused a rapid channel mixing in the temperature range of $T \leq 20 \mathrm{~K}$. 


\subsubsection{Temperature Dependence of $L_{\phi}$ and Decay Mechanism}

The phase coherence length is another fitting parameter in the HLN formulation of magnetoconductance and it usually decreases with increasing temperature. The decrease in the phase coherence length is due to electron- electron scattering below $20 \mathrm{~K}$. In order to explain the phase relaxation mechanism in WAL, we systematically investigated the dependence of phase coherence length for our pure and Mn doped samples. For electron-phonon (e-ph) scattering, the relation between phase coherence length and temperature is given as, $L_{\phi} \approx T^{-1}$ for electron-transverse phonon, and similarly $L_{\phi} \approx T^{-1.5}$ for electron-longitudinal phonon interactions [107]. Another mechanism consists of electron electron (e-e) collisions with small energy transfers [25]. For the e-e dominated scattering, the temperature dependence must yield the relations $L_{\phi} \approx T^{-0.25}, L_{\phi} \approx T^{-0.5}$ and $L_{\phi} \approx T^{-0.75}$ in $1 \mathrm{D}, 2 \mathrm{D}$, and $3 \mathrm{D}$ respectively. Our first observation for the Mn doped samples yields a lower phase coherence length than the pure sample. This is due to the increase in disorder/crystal defects and imperfections formed with Mn doping. Despite the satisfactory power law fits, dephasing in Mn doped samples can not be explained by a simple e-e or e-ph scattering (Fig. 5.7). As Mn doping increases, the phase coherence length decays at a slower rate and the power law decreases. In the $x=0.078$ sample, the power law coefficient coincides with the prediction of 1D e-e scattering theory, with $L_{\phi} \approx T^{-0.25}$. However, this is incorrect because the dimensions of our devices rule out this possibility since $L>>L_{\phi}$ and $W>>L_{\phi}$ with $L / W=2.74$. Temperature dependence of phase coherence length (Fig. 5.7) suggests that the dephasing can not be explained by only e-e scattering in the case of Mn doped thin films. It is not surprising to see that the low temperature region where e-e scattering dominates coincides with the region where one expects to see a correction to resistance in the 

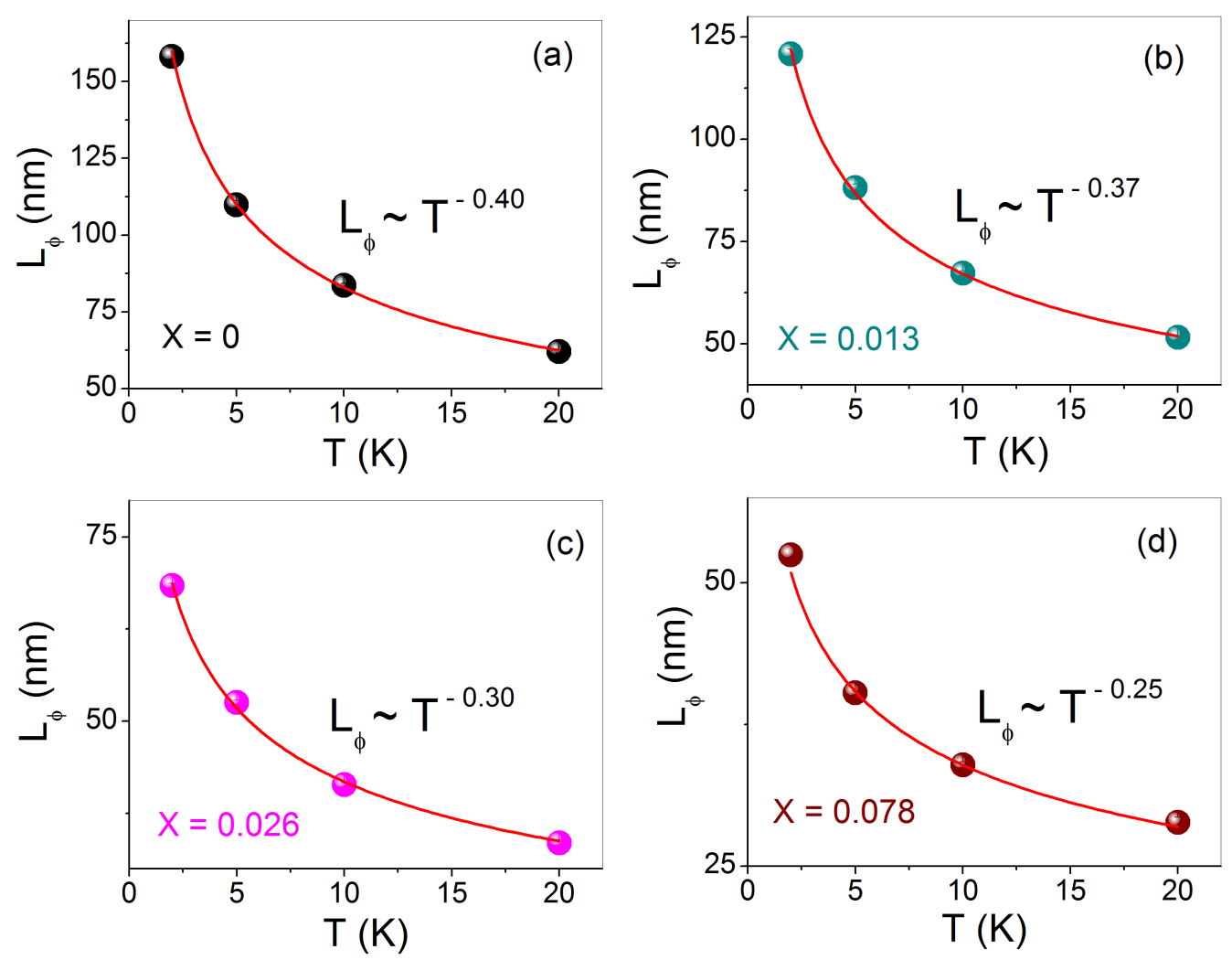

Figure 5.7: Temperature evolution of $L_{\phi}$ for $\mathrm{Bi}_{2-x} \mathrm{Mn}_{x} \mathrm{Se}_{3}$ films. The coefficient "p" in $L_{\phi}$ vs $T^{-p}$ decreases rapidly with increasing $x$, Mn concentration. Red lines represents power law fits to the phase coherence lengths. Power law relations are labeled in each graph (a)-(d), for $x=0,0.013,0.026,0.078$ respectively. Note that highly-doped sample, $x=0.131$ does not show any WAL at high temperatures and therefore is not included in this figure.

form of Kondo effect. Therefore, the Kondo effect as a possible contribution related to the scattering from magnetic impurities can not be ruled out. In fact, the power law coefficient decreases with increasing Mn concentration which clearly indicates scattering by randomly distributed local moments. We also detected a breakdown of WAL effect with increasing Mn as spin scattering also suppresses the quantum interference. In conclusion, temperature dependence of phase coherence length in Mn doped samples suggests that the dominant dephasing mechanism can not be 
explained by e-e or e-ph interaction and it mainly stems from scattering by local moments.

\subsection{Three Channel HLN Fit Results for 12 QL $\mathrm{Bi}_{2-x} \mathbf{M n}_{x} \mathrm{Se}_{3}$ Thin Films}

After analyzing the transport with single channel HLN model, we will analyze the same data for $12 \mathrm{QL} \mathrm{Bi}_{2-x} \mathrm{Mn}_{x} \mathrm{Se}_{3}$ thin films with a three channel-modified HLN formula at $T=2 \mathrm{~K}$. The three channels are: top surface, bulk channel, and bottom surface and all of them are two dimensional at low temperatures. The modified HLN formula can be written as

$$
\Delta \sigma_{\perp}(B)=\sum_{i=1}^{3} \alpha_{i} \frac{e^{2}}{2 \pi^{2} \hbar}\left[\psi\left(\frac{\hbar}{4 e L_{\phi_{i}^{2} B}^{2}}+\frac{1}{2}\right)-\ln \left(\frac{\hbar}{4 e L_{\phi_{i}}^{2} B}\right)\right]
$$

where $\alpha_{i}$ is a constant equal to -0.5 for each $2 \mathrm{D}$ channel, $\psi$ is the digamma function and $L_{\phi i}$ is the phase coherence length. Two surface states were fixed to have similar conductance contributions to total conductance with a prefactor $\alpha_{1}$ (top surface) $=\alpha_{3}$ (bottom surface $)=-0.5$ each, for a total of $\alpha_{s}=-1.0$. The bulk channel has a prefactor of $\alpha_{2}=-0.5$. There are only two fitting parameters in Eq.(5.1): the bulk phase coherence length $\left(L_{\phi b}\right)$ and the total surface phase coherence length $\left(L_{\phi s}\right)$. The magnetoconductance data at $T=2 \mathrm{~K}$ are shown in Fig. 5.8. The shape of the WAL feature smeared out for the Mn doping range of $x \geq 0.026$, thus it puts a limit for the surface contribution to the transport. Interestingly, from the fit results to the three-channel HLN formula, we obtained $L_{\phi s} \approx 0$ for the samples with $x=0.078$ and $x=0.131$ confirming the validity of our model (Fig. 5.8b). The pure and low-doped samples yielded almost a constant surface phase coherence length 

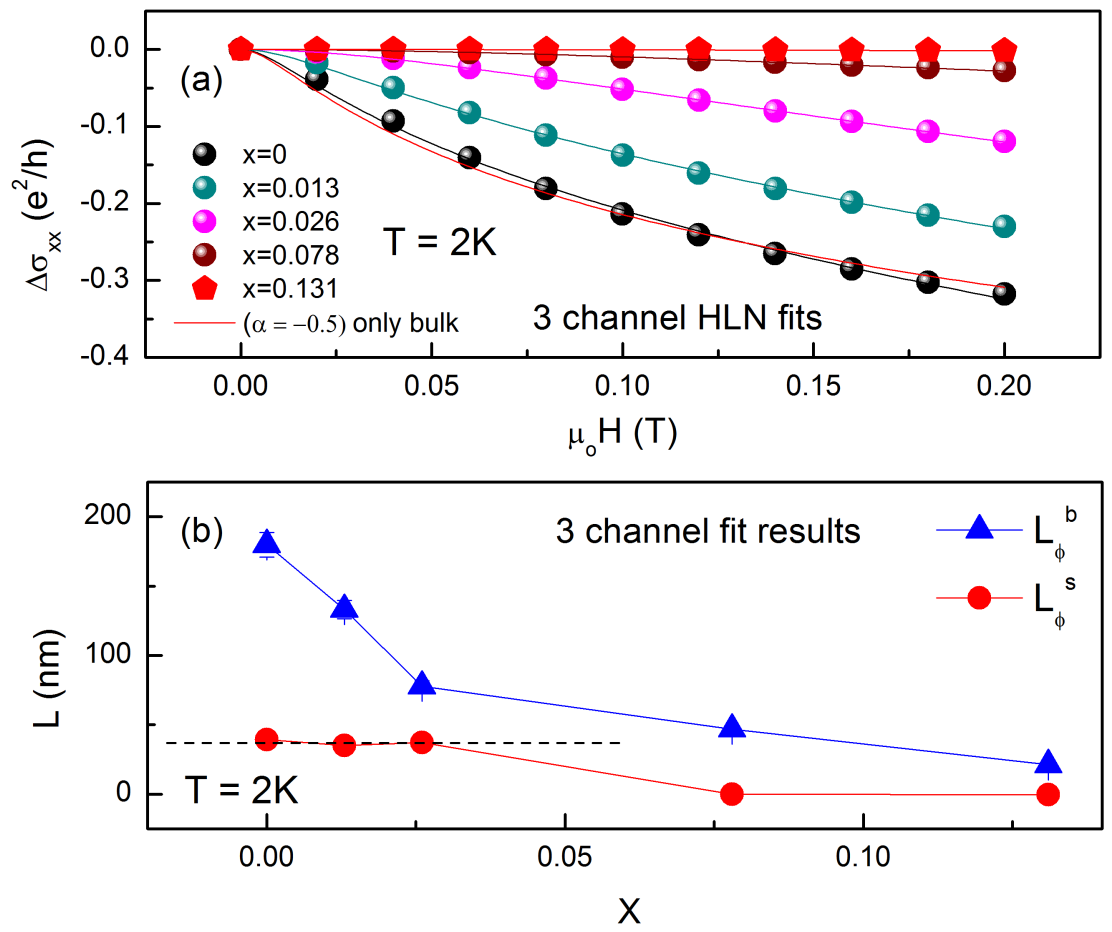

Figure 5.8: (a) Magnetoconductance (MC) data and HLN formula fits for 12 QL $\mathrm{Bi}_{2-x} \mathrm{Mn}_{x} \mathrm{Se}_{3}$ films at $T=2 \mathrm{~K}$. Solid lines represent the fits to the three channel HLN equation. Red line is a fit performed on the pure sample's MC data with only bulk channel, which is not satisfactory. Note: The shape of the MC curvature starts to change at $x=0.026$ sample indicating the bulk starts to dominate the transport completely above this concentration. (b) Coherence lengths $L_{\phi b}$ and $L_{\phi s}$ vs. $x$. Green line indicates a constant value of $L_{\phi s} \approx 37 \mathrm{~nm}$ in pure and lowly-doped samples.

indicating that the surface states are robust against small doping concentrations. On average, $L_{\phi s} \approx 37 \mathrm{~nm}$ consistent with our other samples. Bulk phase coherence length $\left(L_{\phi b}\right)$ decreased with increasing Mn concentration due to increasing scattering from magnetic impurities (Fig. 5.8b).

We also tried to fit the pure sample's MC data with only a bulk contribution by fixing the surface contribution to zero $\left(\alpha_{s}=0.0\right)$. Figure 5.8a (red solid line) shows the corresponding fit, and suggests that there is more than single channel 
in the transport, which is consistent with the existence of independent conducting channels.

\subsection{Conclusions}

A simplified, single channel HLN model was used to compare the contributions of bulk and surface states to the transport. The single channel HLN model revealed that surface and bulk conducting channels were mixed and acted as single entity in pure and minimally doped samples. A channel separation in the intermediate doping regime was observed. Increasing Mn concentration yielded very small phase coherence lengths for highly-doped samples at $T=2 \mathrm{~K}$. Despite the single channel model capturing the correct behavior of a decreasing phase coherence length with increasing Mn doping, it failed to explain the contribution coming from bulk and surface states independently. On the other hand, the contributions coming from the bulk and surface channels to the transport were obtained independently, by using a three-channel HLN model. In the low doping regime, surface phase coherence length $\left(L_{\phi s}\right)$ was not affected, until the Mn concentration reached to $x=0.078$ in $\mathrm{Bi}_{2-x} \mathrm{Mn}_{x} \mathrm{Se}_{3}$. We extracted an average of $L_{\phi s} \approx 37 \mathrm{~nm}$, which was almost constant for pure and lowly-doped samples. Due to the increasing scattering from Mn impurities, bulk phase coherence length $\left(L_{\phi b}\right)$ decreased as a function of Mn concentration. Our modified HLN formula for three independent channels gave satisfactory fits and captured the transport properties of both pure and Mn doped samples discussed in this chapter. A more extended discussion on this modified HLN model will be given by combining it with the Altshuler-Aronov formalism of parallel transport and electron electron interaction (EEI) in the next chapter. 


\section{Chapter 6}

\section{Low Temperature Transport}

\section{Properties of $\mathrm{Bi}_{2} \mathrm{Se}_{3}$ Thin Films}

\subsection{Introduction}

The surface and bulk electronic transport properties of topological insulator $\mathrm{Bi}_{2} \mathrm{Se}_{3}$ thin films in parallel and perpendicular magnetic fields. $\mathrm{Bi}_{2} \mathrm{Se}_{3}$ thin films with nominal thickness values of 12, 16, 20 and 25 quintuple layers (QLs) were grown by molecular beam epitaxy (MBE). Structural data indicated a slight increase in the disorder with increasing film thickness, but overall the samples were highly crystalline. Weak antilocalization (WAL) was observed in magnetoconductance measurements with the magnetic field applied parallel to the samples' surface and at temperatures between $2 \mathrm{~K}$ and $20 \mathrm{~K}$, which indicated a significant contribution to the transport from the bulk states. Large phase coherence lengths with the field applied both perpendicular and parallel to the surface confirmed the diffusive nature of the transport. Quantitatively, the phase coherence lengths $\left(L_{\phi}\right)$ obtained from the Altshuler-Aronov and Hikami-Larkin-Nagaoka mechanisms were an order of magnitude larger than the 
film thickness $(d)$ for all of the samples $\left(L_{\phi}>>d\right)$. The dephasing mechanism was determined from the temperature evolution of phase coherence length and indicated electron-electron scattering as the main contribution to the dephasing mechanism below 20K. Parallel magnetoconductance and the corresponding bulk phase coherence lengths were used to estimate the contribution of bulk and surface states in the perpendicular transport. Temperature dependence of conductivity was dominated by electron electron interaction (EEI) at low temperatures. We observed an increase

in Coulomb screening parameter, $\widetilde{F}$ in the bulk channel with increasing film thickness which indicated that the effective EEI is strongest in the thinnest films.

\subsection{Sample Preparation and Experimental Meth- ods}

$\mathrm{Bi}_{2} \mathrm{Se}_{3}$ thin films with a nominal thickness of $12,16,20$, and 25 QLs were grown on $\mathrm{Al}_{2} \mathrm{O}_{3}$ (0001) single crystal substrates via molecular beam epitaxy (MBE) using high purity (\% 99.999) Bi, and Se. The films were grown on double-sided polished $\mathrm{Al}_{2} \mathrm{O}_{3}$ (0001) pieces which yielded a better film quality than the ones grown on single-sided polished $\mathrm{Al}_{2} \mathrm{O}_{3}$ (0001). Prior to growth, substrates were annealed in air for 2 hours at $1200{ }^{\circ} \mathrm{C}$ to achieve an atomically smooth surface. Two step growth was implemented [54] with a substrate temperature of $T_{s}=140{ }^{\circ} \mathrm{C}$ for the first three quintuple layers and, $T_{s}=275{ }^{\circ} \mathrm{C}$ for the remaining layers of the film. The growth of $\mathrm{Bi}_{2} \mathrm{Se}_{3}$ is typically done in Se rich conditions to compensate the desorption of $\mathrm{Se}$ atoms during the ablation process. A molecular beam ratio of $\mathrm{Se} / \mathrm{Bi} \approx 15$ was used in growing our films. Growth rate was determined by Bi flux with a rate of 0.6 QL/min. The film thickness were chosen as $d \geq 12$ QL to avoid band hybridization 

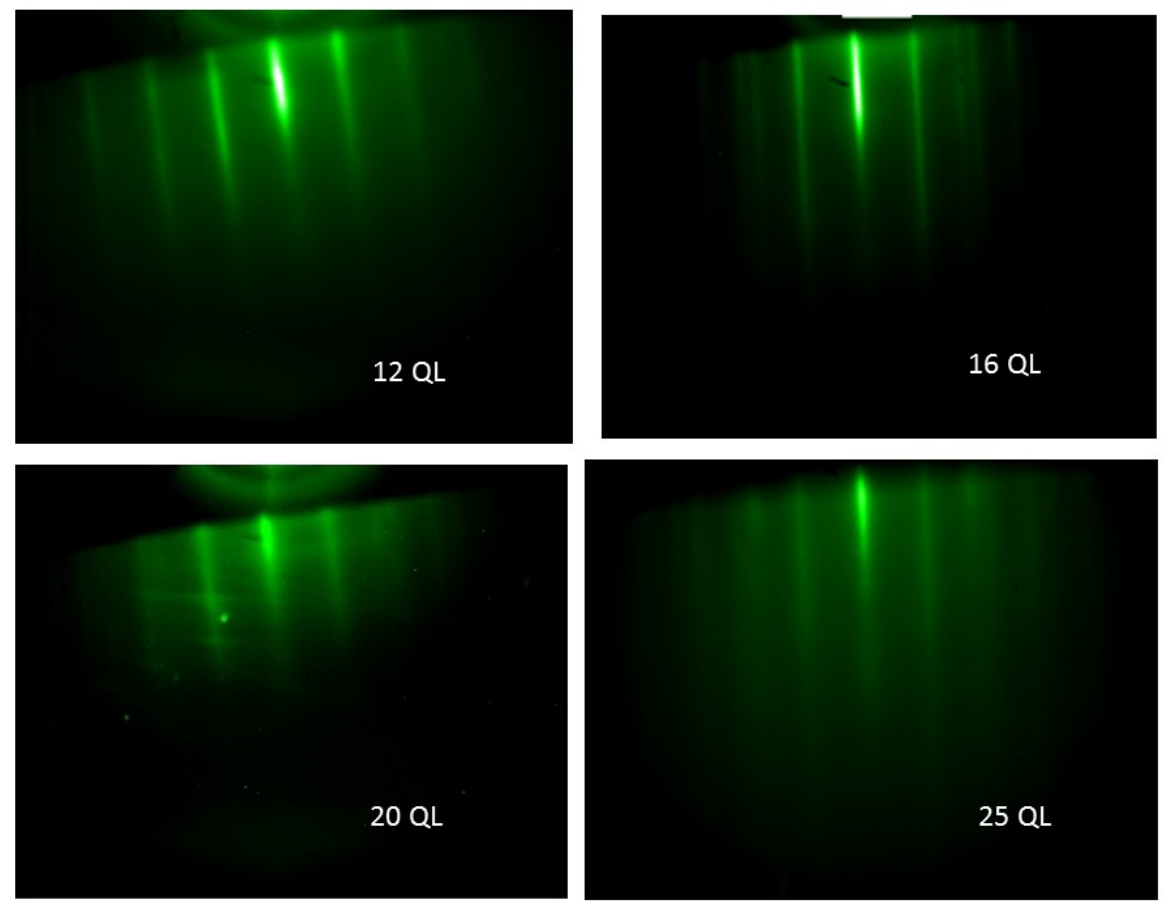

Figure 6.1: Streaky RHEED images showing a highly epitaxial $\mathrm{Bi}_{2} \mathrm{Se}_{3}$ films with thickness of 12, 16, 20 and 25 QLs.

(direct coupling) between top and bottom surfaces which is typically seen in ultrathin films with a thickness less than 6 QL [108]. During the growth, surface quality of the films was monitored by reflection high energy electron diffraction (RHEED). Figure 6.1 shows RHEED images for each sample which were taken at the end of the growth. Streaky RHEED pattern indicated the formation of epitaxial and smooth films. Also, RHEED oscillations for each sample is shown in Fig. 6.2. The oscillations corresponding the low temperature growth of first $3 \mathrm{QL}$ was not captured due the amorphous nature of the films. RHEED oscillations corresponding to a completion of a QL were also detected for all of the other films. The 25 QL sample which is the thickest among the others, showed RHEED oscillations until 8 QL, which indicated an increasing roughness for this film.

X-ray diffraction (XRD) and x-ray reflectivity (XRR) measurements were per- 

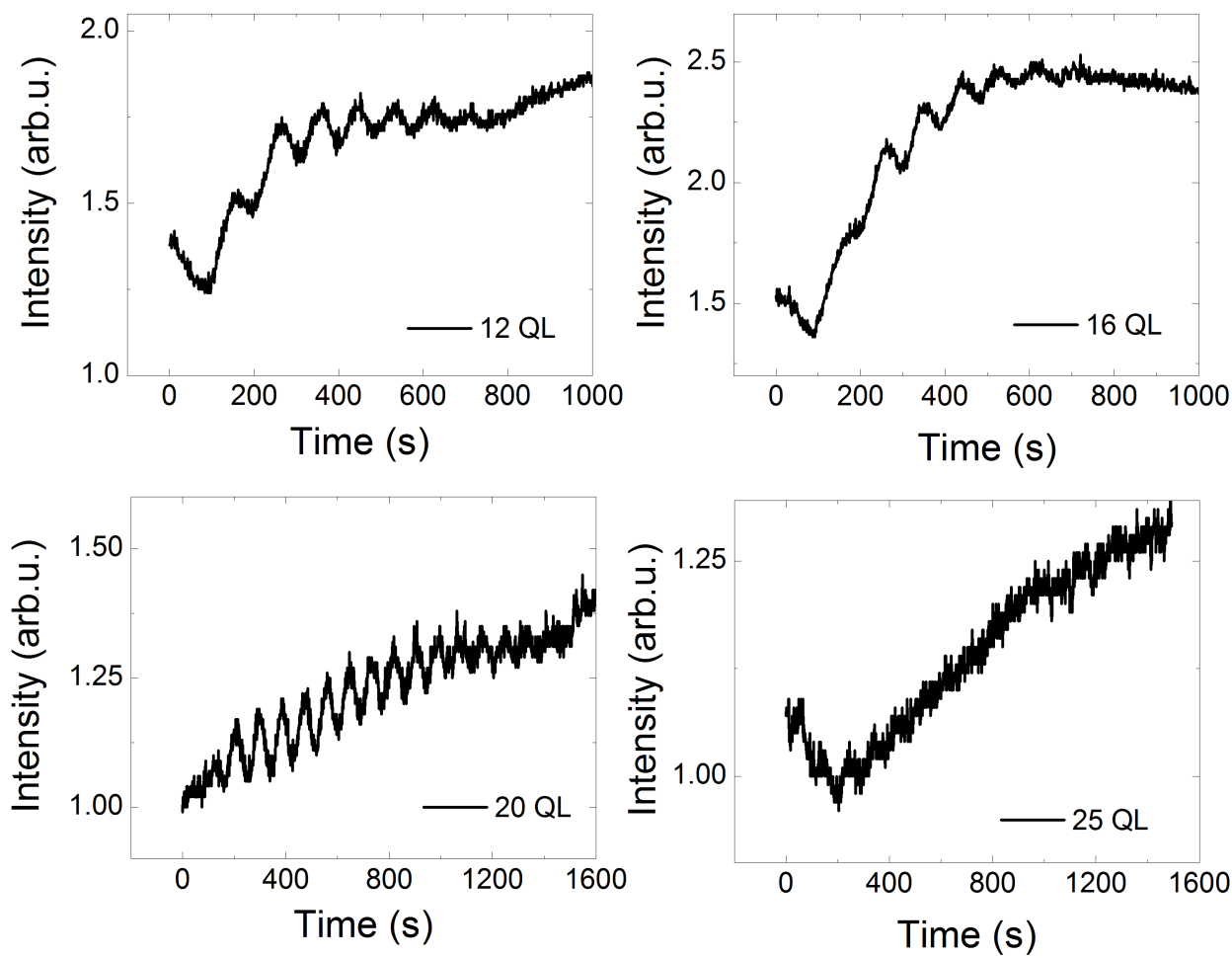

Figure 6.2: RHEED oscillations for 12-25 $\mathrm{QLs} \mathrm{Bi}_{2} \mathrm{Se}_{3}$ films. Each peak corresponds to a completion of a quintuple layer $(\mathrm{QL})$. The oscillations for the first $3 \mathrm{QL}$ were not recorded at the low temperature step of the growth.

formed using $\mathrm{CuK} \alpha$ radiation with a wavelength of $0.15418 \mathrm{~nm}$. The layered rhombohedral crystal structure of $\mathrm{Bi}_{2} \mathrm{Se}_{3}$ was confirmed with the observation of $(00 l)$ reflections (Fig. 6.3a). In order to investigate the disorder and defect density, rocking curve scans of $\mathrm{Bi}_{2} \mathrm{Se}_{3}$ (0015) reflections were recorded which revealed an increase in the disorder level with increasing film thickness (Fig. 6.3b and inset). Figure 6.3c shows the XRR data and the corresponding fits for all of the films. The agreement between the data and the fits was excellent and nearly all finite size Kiessig fringes were captured until the first Bragg peak. XRR data was fitted with GenX [59] with the parameters shown in Table 6.1. The thicknesses of the films were confirmed to be within $\% 5$ to the in-situ values on average. The surface roughness on the top 
of $\mathrm{Bi}_{2} \mathrm{Se}_{3}$ layer was extracted from these XRR fits and analysis. Roughness values were $0.37,0.47,0.61$ and $1.67 \mathrm{~nm}$ for 12, 16, 20 and 25 QL samples, respectively. The increase in roughness with film thickness is consistent with the increase in the width of the rocking curve scans of $\mathrm{Bi}_{2} \mathrm{Se}_{3}$ (0015) reflection (inset to Fig. 6.3a). A detailed discussion on film thickness and roughness determination can be found elsewhere $[60,100]$. Transport measurements were performed in a physical property measurement system (PPMS) with six-probe Hall bar configuration. The magnetic field was applied perpendicular and parallel to film surface. In both configurations, the magnetic field was kept perpendicular to current direction.

\subsection{HLN Formalism of Perpendicular Magneto- conductance}

Weak antilocalization of topological insulators results from the strong spin orbit coupling and protected by time reversal symmetry in the absence of magnetic perturbation. The absence of backscattering of time reversed electron paths causes the magnetoconductance to reach a maximum. The application of magnetic field introduces an arbitrary Aharonov-Bohm phase into the system and increases backscattering which results in a sharp decrease in the magnetoconductance. For a diffusive system with spin orbit interaction, 2D magnetoconductance was studied by Hikami, Larkin and Nagaoka (HLN) formalism. The quantum correction to the Drude conductivity is given by HLN equation [24];

$$
\Delta \sigma_{\perp}(B)=\sum_{i=1}^{3} \alpha_{i} \frac{e^{2}}{2 \pi^{2} \hbar}\left[\psi\left(\frac{\hbar}{4 e L_{\phi_{i}}^{2} B}+\frac{1}{2}\right)-\ln \left(\frac{\hbar}{4 e L_{\phi_{i}}^{2} B}\right)\right]
$$



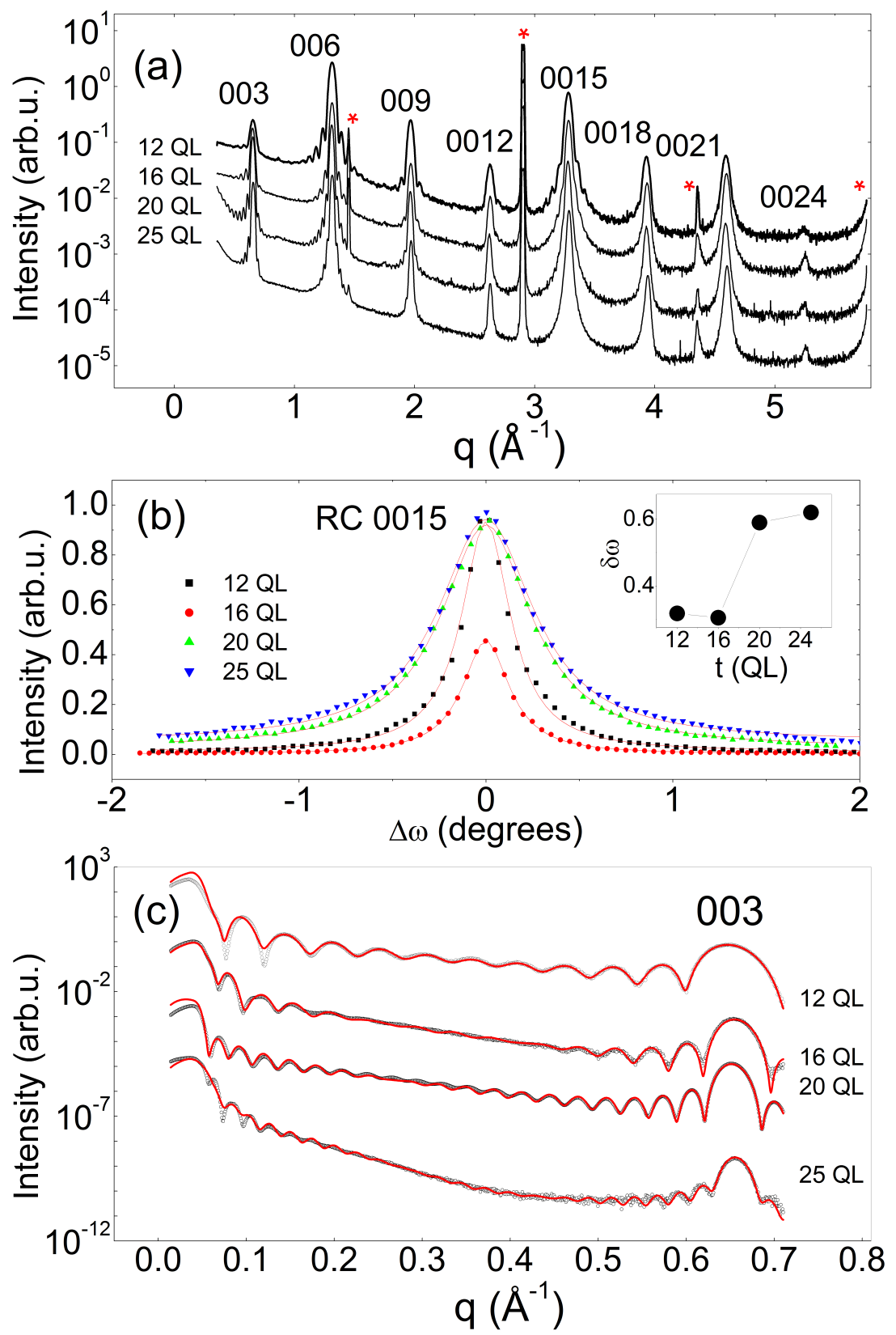

Figure 6.3: (a) X-ray diffraction (XRD) data for $\mathrm{Bi}_{2} \mathrm{Se}_{3}$ films revealing (003) family of planes, and confirming the growth is along the c-axis. Asterisks represent the peaks from the substrate. (b) Rocking curve scan for the $\mathrm{Bi}_{2} \mathrm{Se}_{3}$ (0015) reflection. Inset: FWHM of rocking curve indicating an increase in disorder with the film thickness. (c) X-ray reflectivity (XRR) data (circles) for all the films used in this study. Red lines represents the fits to the XRR data. The first Bragg peak of $\mathrm{Bi}_{2} \mathrm{Se}_{3}$ (003) is labeled in figure at $q=0.65 \AA^{-1}$. 

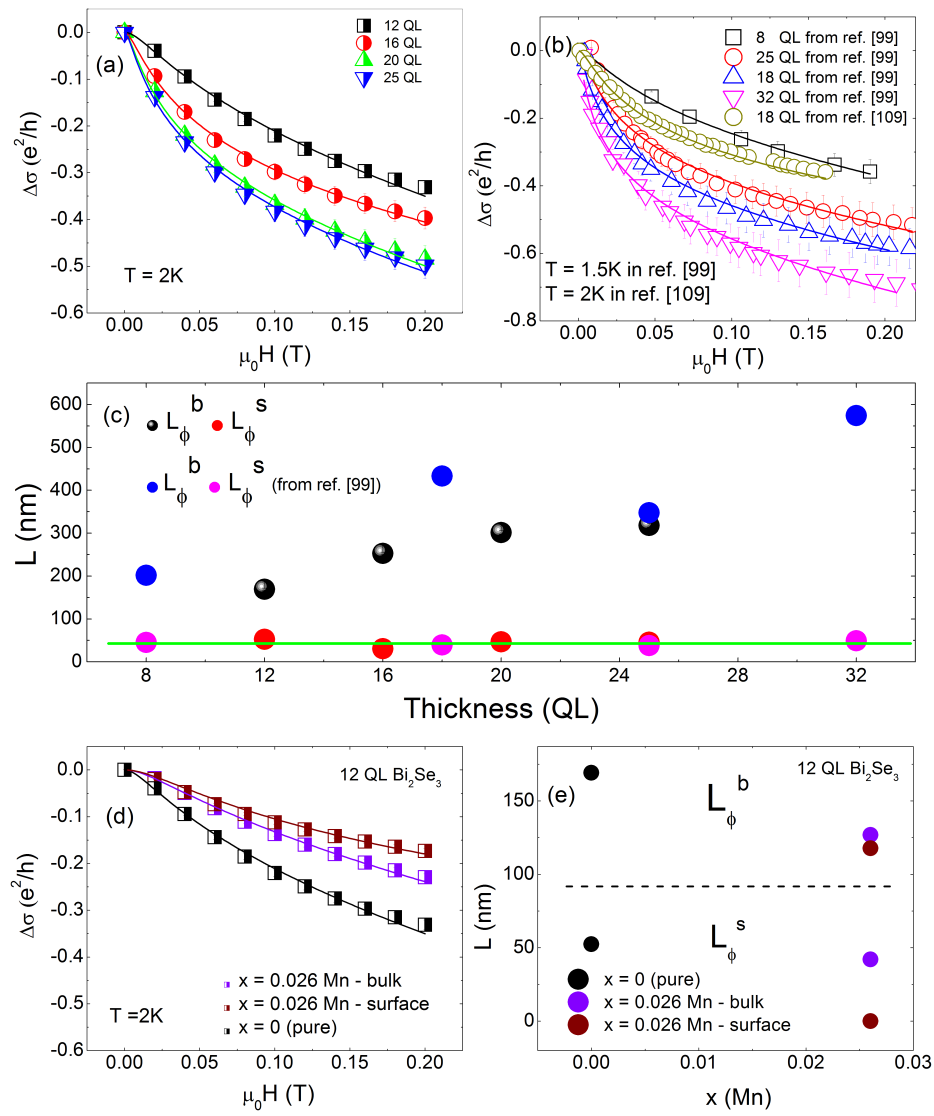

Figure 6.4: (a) HLN formula fits to the magnetoconductance at low fields for $\mathrm{Bi}_{2} \mathrm{Se}_{3}$ films with different thickness. Solid red lines represent the fits (b) Digitized magnetoconductance data from references [99] and [109]. $10 \%$ error bar included in the data from ref. [99] as reported by authors. Lines represents the HLN formula fits (c) The phase coherence lengths for the bulk $\left(L_{\phi b}\right)$ and surface $\left(L_{\phi s}\right)$ vs. film thickness. Black and red circles represents the phase coherence lengths for bulk and surface, respectively for our samples. Similarly, blue and magenta circles represents the bulk $\left(L_{\phi b}\right)$ and surface $\left(L_{\phi s}\right)$ phase coherence lengths obtained from HLN fits for the samples in ref. [99]. Green line indicates and average of $L_{\phi s} \approx 43 \mathrm{~nm}$ in a wide thickness range including our samples and the samples from reference [99]. Note : The data in reference [109] gives satisfactory fits with only bulk phase coherence lengths, indicating that the measurements were dominated by bulk states. (d) Magnetoconductance data for $12 \mathrm{QL} \mathrm{Bi}_{2} \mathrm{Se}_{3}$ samples with pure and with $x=0.026$. Lines represent the fits to three channel HLN formula (e) Phase coherence lengths for Mn doped samples. The sample with $x=0.026, \mathrm{Mn}$ (surface doping) shows negligibly small value for the $L_{\phi s}$ (brown circles). Dashed line is a guide to the eye in order to separate the regions for $L_{\phi b}$ and $L_{\phi s}$. 


\begin{tabular}{|c|c|c|c|}
\hline Sample & Layer & Thickness $(\mathrm{nm})$ & Roughness $(\mathrm{nm})$ \\
\hline $12 \mathrm{QL}$ & $\mathrm{MgF}_{2}$ & 7.21 & 1.73 \\
& Top interface & 0.58 & 0.31 \\
& $\mathrm{Bi}_{2} \mathrm{Se}_{3}$ & 11.4 & 0.37 \\
& Bottom interface & 0.50 & 0.17 \\
& $\mathrm{Al}_{2} \mathrm{O}_{3}$ & & 0.11 \\
\hline $16 \mathrm{QL}$ & $\mathrm{MgF}_{2}$ & 9.61 & 1.49 \\
& Top interface & 1.16 & 0.37 \\
& $\mathrm{Bi}_{2} \mathrm{Se}_{3}$ & 15.9 & 0.47 \\
& Bottom interface & 0.87 & 0.43 \\
& $\mathrm{Al}_{2} \mathrm{O}_{3}$ & & 0.09 \\
\hline $20 \mathrm{QL}$ & $\mathrm{MgF}_{2}$ & 7.05 & 2.21 \\
& Top interface & 0.25 & 0.12 \\
& $\mathrm{Bi}_{2} \mathrm{Se}_{3}$ & 21.4 & 0.61 \\
& Bottom interface & 1.28 & 0.11 \\
& $\mathrm{Al}_{2} \mathrm{O}_{3}$ & & 0.03 \\
\hline $25 \mathrm{QL}$ & $\mathrm{MgF}_{2}$ & 13.3 & 1.94 \\
& Top interface & 1.17 & 0.31 \\
& $\mathrm{Bi}_{2} \mathrm{Se}_{3}$ & 25.2 & 1.67 \\
& Bottom interface & 1.03 & 0.26 \\
& $\mathrm{Al}_{2} \mathrm{O}_{3}$ & & 0.11 \\
\hline
\end{tabular}

Table 6.1: Thickness and roughness parameters from fitting the XRR data with GenX [59]. The thickness of $\mathrm{Bi}_{2} \mathrm{Se}_{3}$ layers agrees well with the in-situ values to within $5 \%$. The surface roughness of $\mathrm{Bi}_{2} \mathrm{Se}_{3}$ layer increases with increasing film thickness

where $\alpha_{i}$ is a constant equal to -0.5 for each $2 \mathrm{D}$ channel, $\psi$ is the digamma function and $L_{\phi i}$ is the phase coherence length. It must be noted that HLN formalism of perpendicular magnetoconductance and Altshuler-Aronov formalism of parallel transport are both studied in the low field regimes. Since the Zeeman g-factor of $\mathrm{Bi}_{2} \mathrm{Se}_{3}$ is quiet large, (23 when $H$ is in plane, and 32 when $H$ is out of plane) [110], Zeeman splitting of spin states becomes important in larger fields. Therefore, magnetic field must lie in the range of $H \leq k T / g \mu_{B}$. We assumed the presence of three parallel conduction channels in the perpendicular transport since bulk states also 
possess WAL. Two surface states were assumed to have similar conductance contributions to total conductance with a prefactor $\alpha_{1}$ (top surface) $=\alpha_{3}$ (bottom surface) $=-0.5$ each, with a total of $\alpha_{s}=-1.0$. The bulk channel was assumed to have prefactor of $\alpha_{2}=-0.5$. There are only two fitting parameters in Eq.(6.1): the bulk phase coherence length $\left(L_{\phi b}\right)$ and the total surface phase coherence length $\left(L_{\phi s}\right)$.

The results of the measurements and the corresponding fits at $T=2 \mathrm{~K}$ are shown in Fig. 6.4. By fitting the magnetoconductance shown in Fig. 6.4a, we extracted $L_{\phi b}$ and $L_{\phi s}$. For truly 2D surface states, the surface phase coherence length $\left(L_{\phi s}\right)$ should be independent of thickness. The phase coherence lengths obtained by this procedure are plotted in Fig. 6.4c. In the thickness range of 12-25 QLs, $L_{\phi b}$ increased with increasing thickness due to the quasi 2D nature of bulk states. The increase in $L_{\phi b}$ clearly indicates that there is a finite thickness effect for the electron trajectories that resides in the bulk of $\mathrm{Bi}_{2} \mathrm{Se}_{3}$ films. Surface phase coherence lengths are also plotted in Fig. 6.4c, which shows nearly a constant value over the same thickness range. The constant value over a wide range of thickness values confirms that our modified HLN model captures the physical nature of transport in $\mathrm{Bi}_{2} \mathrm{Se}_{3}$ films. The average value of $L_{\phi s}$ for our samples was approximately $43 \mathrm{~nm}$. Another interesting observation from the fit results was identifying the relative strengths of bulk and surface contributions to the transport. Because of the finite thin film thickness, the bulk states act as a quasi-2D system which can also have WAL (this is assumed in the modified HLN model). Our findings are consistent with the bulk dominated transport with a higher values of $L_{\phi b}$ than $L_{\phi s}\left(L_{\phi b}>L_{\phi s}\right)$. To demonstrate the validity of the model, we digitized the data in Refs. [99] and [109] and fitted the corresponding magnetoconductance as shown in Fig. 6.4b. The fit results for the digitized data from these references are plotted in Fig. 6.4c together with the results for our samples. 

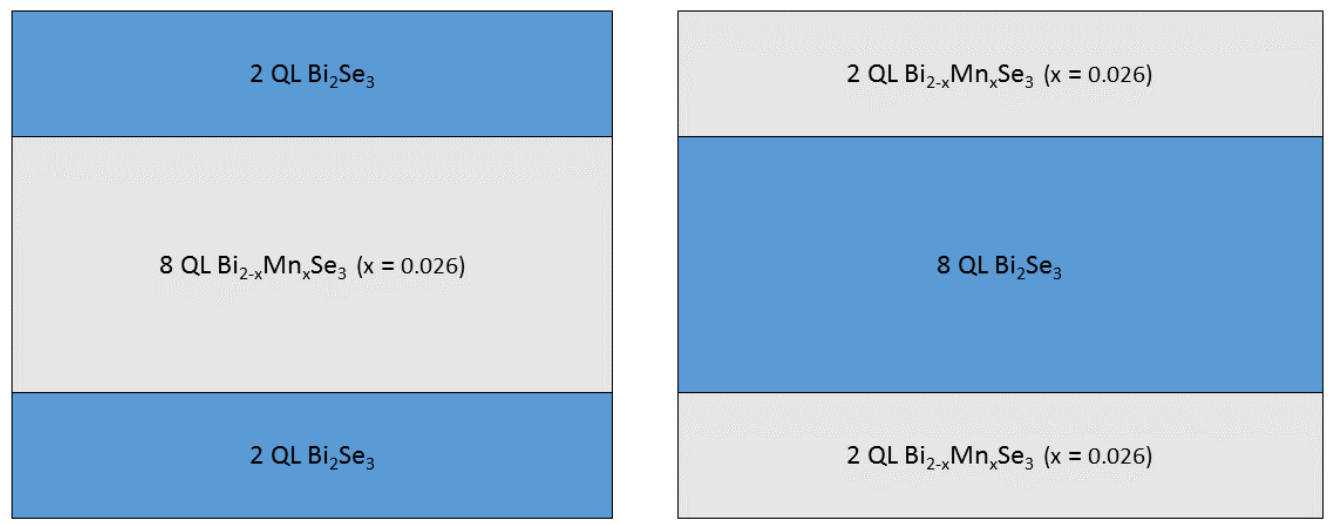

Figure 6.5: Left: Bulk doping of $\mathrm{Mn}$ in the $8 \mathrm{QL}$ of a $12 \mathrm{QL} \mathrm{Bi}_{2-x} \mathrm{Mn}_{x} \mathrm{Se}_{3}$ film with $x=0.026$. Blue and gray regions indicate pure $\mathrm{Bi}_{2} \mathrm{Se}_{3}$ and $\mathrm{Mn}$ doped $\mathrm{Bi}_{2-x} \mathrm{Mn}_{x} \mathrm{Se}_{3}$ (with $x=0.026$ ) layers, respectively. Right: Surface doping of Mn in the top+bottom 2 QLs of $\mathrm{Bi}_{2-x} \mathrm{Mn}_{x} \mathrm{Se}_{3}$ film. 8 QL thick bulk is a pure $\mathrm{Bi}_{2} \mathrm{Se}_{3}$ film.

It is obvious that the $L_{\phi s}$ shows a constant value over the thickness range for 8-32 QLs thin films studied in reference [99]. The average surface phase coherence length is, $L_{\phi s} \approx 42 \mathrm{~nm}$, very close to the value that we have for our samples. On the other hand, $L_{\phi b}$ increases with the thickness, again indicating the thickness dependence of bulk phase coherence length. However, the data from Ref. [109] were easily fitted with only $L_{\phi b}$, that is, the bulk states dominate the transport. As a representative sample, 18 QL data was digitized from ref. [109] which is shown in Fig. 6.4b. We obtained $L_{\phi b} \approx 264 \mathrm{~nm}$ and $L_{\phi s} \approx 0$ which confirms that the measurements is only dominated by the bulk channel.

To further test the validity of our modified HLN model, two 12 QL thick $\mathrm{Bi}_{2-x} \mathrm{Mn}_{x} \mathrm{Se}_{3}$ films with Mn concentration of $x=0.026$, were grown in the same conditions as the pure samples. The structure of these two samples are illustrated in Fig. 6.5. The first one of these samples had Mn everywhere except the top+bottom 2 QLs (Fig. 6.5 , left), while the second one had $\mathrm{Mn}$ in the top+bottom 2 QLs of $12 \mathrm{QL} \mathrm{Bi}_{2} \mathrm{Se}_{3}$ 
film (Fig. 6.5, right). The modified HLN model would predict negligible contribution to the transport from the surface state with the Mn doped only in the surface layers. Figures $6.4 \mathrm{~d}$ and $6.4 \mathrm{e}$ show the magnetoconductance data and the fit results obtained at $T=2 \mathrm{~K}$ for these Mn doped samples. The sample which had Mn impurities on the surface layers yielded negligibly small value for surface phase coherence length $\left(L_{\phi s} \approx 0\right)$ (Fig. 6.4e), as expected from the model. The magnetic impurities on the surface states break time reversal symmetry and quench the electronic phase coherence, which results in a bulk dominated transport. However, in the sample which had Mn impurities in the bulk, surface channel contribution to transport survived and the fit yielded a surface phase coherence length of $L_{\phi s} \approx 42 \mathrm{~nm}$, which agrees with the values we have in our undoped samples. Moreover, bulk phase coherence length of Mn doped sample (bulk doped) was smaller than our pure samples' bulk phase coherence length, again confirming that the modified HLN model can distinguish the surface from the bulk transport contributions.

We also studied the temperature dependence of bulk phase coherence length and observed a decrease with increasing temperatures as shown in Fig. 6.6. The decrease in the phase coherence length is likely due to the electron electron scattering below $20 \mathrm{~K}$. As the temperature is increased, the Fermi distribution is broadened around $E_{F}$ and leads to an increase in the phase space. The more accessible states in the phase space leads larger number of electrons with energy higher than $E_{F}$. As a result, electron electron scattering increases. At higher temperatures $(T \geq 20 K)$ other types of scattering, such as thermally activated electron-phonon interaction must be considered.

In order to understand the phase relaxation mechanism in WAL, the temperature dependence of phase coherence length was investigated. It has been known 


\begin{tabular}{|ccccccccc|}
\hline Sample & $\begin{array}{c}n_{b} \\
10^{19} \mathrm{~cm}^{-3}\end{array}$ & $\begin{array}{c}n_{s} \\
10^{13} \mathrm{~cm}^{-2}\end{array}$ & $\begin{array}{c}k_{f} \\
\AA^{-1}\end{array}$ & $\begin{array}{c}\mu \\
\mathrm{cm}^{2} / \mathrm{Vs}\end{array}$ & $\begin{array}{c}d_{\text {real }} \\
\mathrm{nm}\end{array}$ & $\begin{array}{c}L_{e} \\
\mathrm{~nm}\end{array}$ & $\begin{array}{c}D \\
\mathrm{~cm}^{2} / \mathrm{s}\end{array}$ & $\begin{array}{c}L_{T} \\
\mathrm{~nm}\end{array}$ \\
\hline $12 \mathrm{QL}$ & 1.85 & 2.10 & 0.11 & 105 & 11.4 & 7.9 & 23 & 94 \\
$16 \mathrm{QL}$ & 2.14 & 3.40 & 0.14 & 366 & 15.9 & 35.1 & 132 & 224 \\
$20 \mathrm{QL}$ & 1.05 & 2.26 & 0.12 & 331 & 21.4 & 25.8 & 79 & 174 \\
$25 \mathrm{QL}$ & 1.25 & 3.16 & 0.14 & 287 & 25.2 & 26.6 & 96 & 191 \\
\hline
\end{tabular}

Table 6.2: Results of typical transport parameters at $T=2 \mathrm{~K}$.

that at low temperatures, electron electron (e-e) scattering dominates the transport in weakly disordered systems. For the e-e dominated scattering, the temperature dependence of phase coherence length must yield $L_{\phi} \approx T^{-0.25}, L_{\phi} \approx T^{-0.5}$ and $L_{\phi} \approx T^{-0.75}$ in $1 \mathrm{D}, 2 \mathrm{D}$, and $3 \mathrm{D}$ respectively. Power law fits to for the bulk phase coherence lengths are plotted in Fig. 6.6a-d. From the results of the fits the bulk phase coherence length scaled with a power law close to -0.5 , and therefore the data suggests $2 \mathrm{D}$ e-e scattering as the main source of dephasing. Despite an increase in the power law exponent with increasing thickness, it did not reach to -0.75 which is the case for $3 \mathrm{D}$ e-e scattering. On the other hand, $L_{\phi s}$ does not behave in the same way as the $L_{\phi b}$, and it appears that $L_{\phi s}$ has a weaker temperature dependence (Fig. 6.6e). The 12 QL thick sample showed a huge loss in the surface phase coherence length and possibly as a result of the rapid channel mixing with increasing temperatures for the thinnest film.

The n-type behavior of conductivity was confirmed from the Hall resistivity data, which were similar to the data discussed in Chapter 5 (Fig. 5.3a). From the Hall effect and magnetoresistance measurements at $T=2 \mathrm{~K}$, we estimated the standard transport parameters: carrier density and mobility, Fermi wave vector, mean free path, diffusion coefficient and the thermal lengths for our samples. The results of this analysis were summarized in Table 6.2. Carrier densities are calculated from 


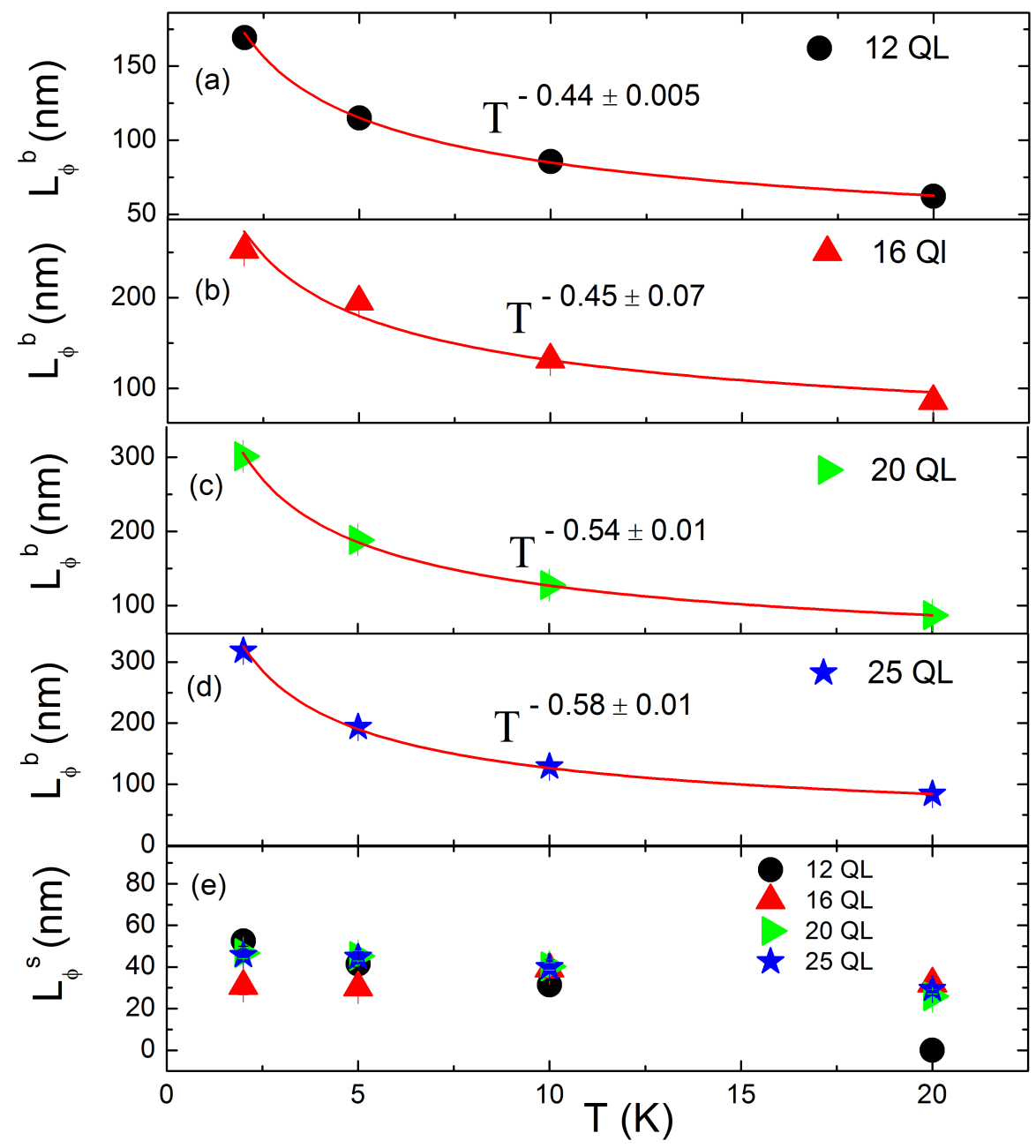

Figure 6.6: Temperature evolution of $L_{\phi b}$ and $L_{\phi s}$ for $\mathrm{Bi}_{2} \mathrm{Se}_{3}$ films. The coefficient 'p' in $L_{\phi b}$ vs. $T^{-p}$ slightly increases with increasing thickness. Red lines represents power law fits to the phase coherence lengths. Power law relations are labeled in each graph (a)-(d), for 12,16, 20, and 25 QL thick films respectively. (e) $L_{\phi s}$ vs. $T$ data. The corresponding changes in $L_{\phi s}$ for 12, 16, $25 \mathrm{QL}$ samples are small. $L_{\phi s}$ for 12 QL sample decreases due to channel coupling with temperature.

the linear slopes of Hall data and corresponding Hall coefficients. Mobilities were calculated from the standard equation, $\mu=1 /\left(\rho_{x x} n_{H} e\right)$ since single type of carrier dominated the transport. The bulk carrier densities and the mobilities were in the range of $1-2 \times 10^{19} \mathrm{~cm}^{-3}$ and $100-400 \mathrm{~cm}^{2} /$ Vs for our films, consistent with the other results reported elsewhere $[54,111]$. The Fermi wavevector was obtained from the 
relation $k_{f}=\sqrt{2 \pi n_{s}}$, where $n_{s}$ is the sheet carrier density. Calculation of Fermi wavevector gave an average of $k_{f} \approx 0.13 \AA^{-1}$ which is in good agreement with the ARPES and transport measurement results [7,54,112]. Mean free paths were obtained from $L_{e}=\hbar k_{f} \mu / e$. The diffusion coefficients obtained from [113] $D=$ $\left(L_{e} * v_{f}\right) / 3$, where the factor $1 / 3$ accounts for three dimensional diffusion and $v_{f}$ is the Fermi velocity calculated from $V_{f}=\hbar k_{f} / m^{*}$. A factor $1 / 3$ was used assuming a three dimensional diffusion since thickness and mean free paths are close in magnitude [113] (see Table 6.2) for the samples investigated here.The effective mass was taken as $m^{*}=0.15 m_{e}$ in these calculations. Finally, thermal lengths were calculated from the diffusion constant via $L_{T}=(\hbar D / k T)^{1 / 2}$ and confirmed that the bulk channel must be treated as a quasi-2D channel due to the large thermal lengths over the film thickness $\left(L_{T}>>d\right)$.

\subsection{Altshuler-Aronov Formalism of Parallel Mag- netoconductance}

The parallel field magnetoconductance (MC) is based on the weak(anti) localization of bulk states and used to probe the bulk conductivity of thin films. Alshuler and Aronov obtained the same result as HLN assuming that electron transport is mostly diffusive in 2D systems with a moderate amount of disorder [25]. The AltshullerAronov model (AA) also predicts that in a strictly 2D film, there must not be any magnetoresistance with the field in the plane. The reason is that in a true $2 \mathrm{D}$ system, scattering can not take place perpendicular to the plane, and therefore the field can not affect the interference of time-reversed paths. However, Altshuler and Aronov predicted that there is a magnetoresistance with $H$ parallel to the surface in thin 
films due to the finite thickness of the film [114]. The WAL in parallel field magnetoconductance is not as pronounced as the perpendicular case since the surface state contribution should be negligible in the parallel field configuration. As the film thickness reduced, a significant decrease in the parallel magnetoconductance is expected due to reduced dimensionality of the bulk states.

We studied MC in parallel fields to understand the bulk properties of $\mathrm{Bi}_{2} \mathrm{Se}_{3}$ thin films. In the AA formalism, the parallel field magnetoconductance of a thin film depends on the thickness $d$ through the formula [113],

$$
\begin{aligned}
\Delta \sigma_{\|}(B)=-\frac{R\left(H_{\|}\right)-R(0)}{R(0) R_{\square}}= & \frac{e^{2}}{2 \pi^{2} \hbar}\left[\frac{3}{2} \ln \left(1+\frac{d^{2} L_{\phi B}^{* 2}}{12 L_{H \|}^{4}}\right)\right. \\
& \left.-\frac{1}{2} \ln \left(1+\frac{d^{2} L_{\phi B}^{2}}{12 L_{H \|}^{4}}\right)+g(T) \ln \left(1+\frac{d^{2} L_{T}^{2}}{12 L_{H \|}^{4}}\right)\right],
\end{aligned}
$$

where the three length scales $L_{\phi b}{ }^{*}, L_{\phi b}$ and $L_{T}$ are spin-orbit, phase coherence and thermal diffusion lengths, respectively. These length scales are related to the diffusion constant of the material by $L_{\phi b}^{*}=\left(D \tau_{\phi b}^{*}\right)^{1 / 2}, L_{\phi b}=\left(D \tau_{\phi b}\right)^{1 / 2}$, and $L_{T}=(\hbar D / k T)^{1 / 2}$, where $\tau$ is the scattering time. Finally, $L_{H}$ is the magnetic length of a particle with charge $2 e$ and related to the magnetic field via $L_{H}=\left(\Phi_{0} / 2 \pi H\right)^{1 / 2}$ where $\Phi_{0}$ is the magnetic flux quantum. It must be noted that for Eq.(6.2) to work, the samples must be in the quasi-two dimensional limit where the thickness satisfies $\lambda \ll d \ll L_{\phi b}, L_{T}$, where $\lambda$ is the electron wavelength. This relation is satisfied for our samples at low temperatures $(T \leq 20 K)$. The first term in Eq.(6.2) is negligible because WAL is dominated by $L_{\phi b}$. Our two-parameter fits on parallel magnetoresistance points to a value of $g(T) \approx 0$ which results in a simplified version of the above formula,

$$
\Delta \sigma_{\|}(B)=\frac{e^{2}}{2 \pi^{2} \hbar}\left[-\frac{1}{2} \ln \left(1+\frac{d^{2} L_{\phi b}^{2}}{12 L_{H \|}^{4}}\right)\right]
$$


where the relations $H \lesssim \Phi_{0} / 2 \pi d^{2}$ and $\Delta \sigma_{\|} / \Delta \sigma_{\perp}=2 d^{2} / L_{T}^{2} \ll 1$ must hold at low fields and temperatures, respectively. Figure 6.7a shows the parallel magnetoconductance data at $T=2 \mathrm{~K}$ for our samples. There is an increase in the quantum correction to the bulk conductivity which confirms the sensitivity of bulk transport to the film thickness. The data were fit to Eq.(6.3) with only one fitting parameter: bulk phase coherence length, $L_{\phi b}$. Bulk phase coherence lengths obtained from parallel and perpendicular transport must agree with each other since bulk channel dominates both measurements. $L_{\phi b}$ as a function of film thickness is shown in Fig. 6.7b. The values for $L_{\phi b}$ obtained for the parallel and perpendicular field configurations agree to within $10 \%$ for the 16, 20 and 25 QLs samples. However, the 12 QL sample yielded $260 \mathrm{~nm}$, which was larger than the value we extracted from the perpendicular measurements. The disagreement for the bulk phase coherence lengths for the thinnest sample may result from another mechanism in the perpendicular transport measurements. In a previous study, it was discussed that indirect coupling between top surface/bulk/bottom surface channels may occur with a thickness up to $10-12$ QLs $[103,115-117]$. The most reasonable explanation for low $L_{\phi b}$ in perpendicular transport is that there is partial indirect coupling between top surface/bulk/bottom surface channels which decreases the electronic phase coherence length due to electrostatic interaction. The possibility of an indirect coupling in the perpendicular transport for $12 \mathrm{QL}$ is consistent with the temperature dependence of $L_{\phi s}$, which indicated a rapid channel mixing (indirect coupling) with increasing temperatures (Fig. 6.6e). The indirect coupling at low temperatures can not be a significant effect for thicker films as the bulk phase coherence lengths in both field configurations were almost the same (Fig. 6.7b) and the changes in $L_{\phi s}$ were very small (Fig. 6.6e). 
In order to compare our results to the perpendicular transport, temperature dependence of bulk phase coherence length was also studied in parallel fields (Fig. 6.7b). The dephasing mechanism was obtained from the temperature dependence of $L_{\phi b}$ assuming a power-law dependence $L_{\phi b} \propto T^{p}$. The data indicated similar power law coefficients to the perpendicular transport for the 16,20, and 25 QL samples, which confirmed 2D electron electron scattering as the main source of coherence loss (Fig. 6.7c-f). A value of $p=0.64 \pm 0.03$ was obtained for the 12 QL sample (Fig. 6.7c), showing a large deviation from the perpendicular transport value suggested an indirect electrostatic interaction between channels with increasing temperature.

\subsection{Temperature Dependence of Conductivity and Electron-Electron Interaction}

The temperature dependence of resistivity measurements in TIs demonstrates an enhancement in the resistivity as the temperature is lowered. This is surprising because the WAL in TIs should cause a decrease in the resistivity at low temperatures. Therefore, it is important to understand the physics behind this resistance anomaly as well as the energy/spin relaxation mechanism at low temperatures. Several studies have been reported to explain low temperature resistance anomaly in 2D conductors in zero and non-zero magnetic fields $[113,118,119]$ with the main physical mechanism attributed to the electron electron interaction (EEI) according to the AA theory. An electron's Coulomb interaction with nearby electrons causes an enhanced scattering and increases the resistance [26] because changes in the charge distribution in a disordered metal can not be screened immediately. Since the electrons are in diffusive regime, they need time to screen the charge distribution. Slow diffusion process leads 

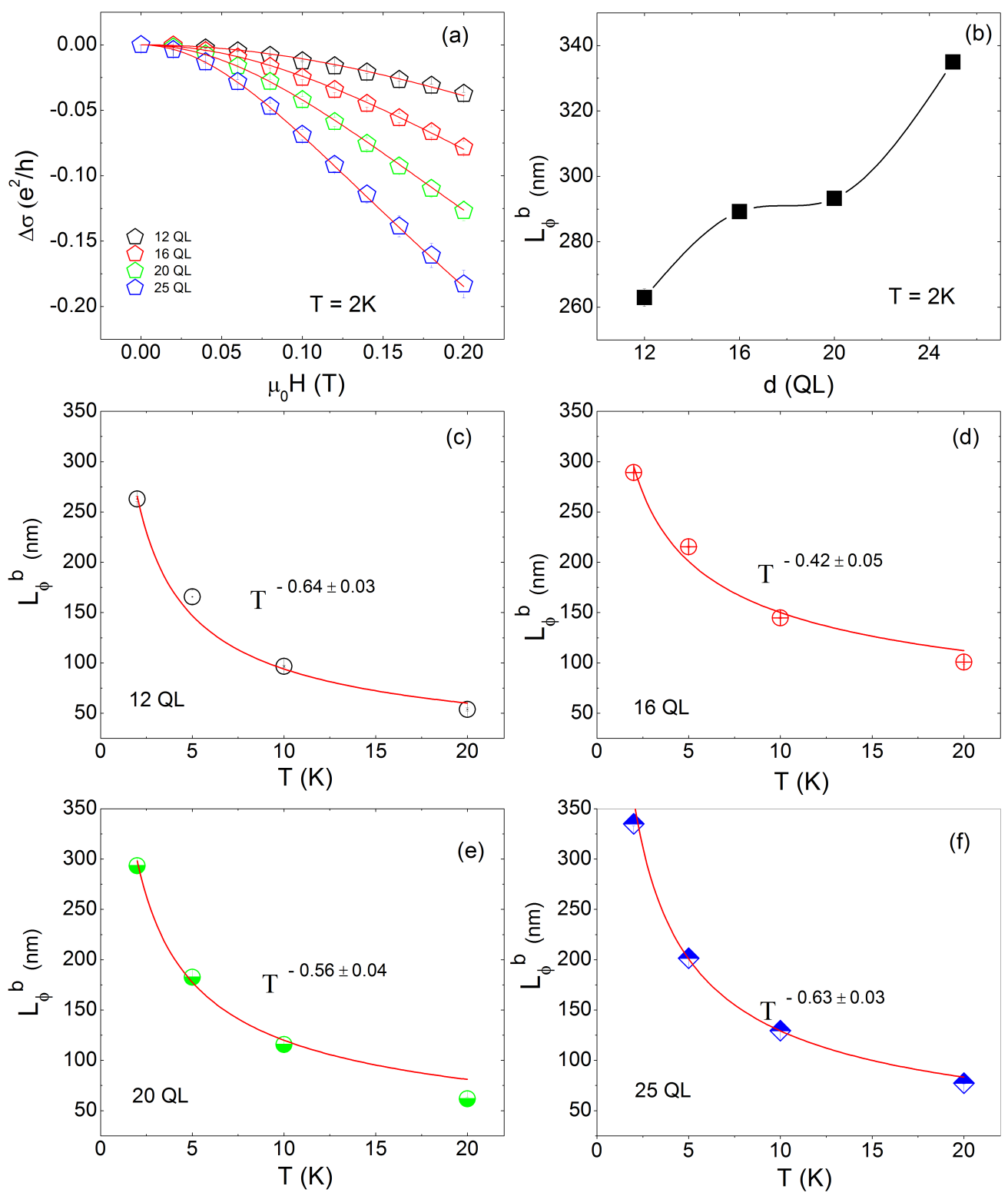

Figure 6.7: (a) Parallel field MC at low fields and at $T=2 \mathrm{~K}$, in units of $e^{2} / h$ for different film thickness. Solid red lines represents the fits to equation (6.3) (b) Bulk phase coherence lengths, $L_{\phi b}$ vs. film thickness. Solid line is a guide to the eye. (c)-(e) $L_{\phi b}$ vs. temperature for 12, 16, 20 and 25 QLs films respectively. Red lines represents the power law fits to the data. Power law coefficients are indicated in each graph. 
a small screening effect for a small electron momentum $q$. As a result, an effective electron electron interaction (EEI) builds up due to the exchange interaction between electron pairs [27]. This brings a correction to the resistance of the system at low temperatures. The correction to the resistance at low temperatures is given by [113]

$$
\frac{R(T)-R_{\min }}{R_{\min }}=|A| \frac{e^{2} R_{\square}}{2 \pi^{2} \hbar} \ln \left(T / T_{0}\right)
$$

where $\mathrm{R}_{\min }$ is the resistance minimum, $\mathrm{R}_{\square}$ is the sheet resistance, and $A=1-$ $\widetilde{F}-p / 2$. The constant 1 is due to the disorder-enhanced EEI and $p=1$ related to the dephasing of phase coherence time and depends on the collision mechanism $\left(L_{\phi} \approx T^{-0.5}\right.$ yields $\tau_{\phi} \approx T^{-1}$ since $\left.L_{\phi}=\left(D \tau_{\phi}\right)^{1 / 2}\right) . \quad \widetilde{F}$ is a parameter reflecting the strength of dynamically screened Coulomb interaction. $T_{0}$ is a characteristic extracted from a linear fit to $\Delta R / R_{\min }$ vs. $\ln \left(T / T_{\circ}\right.$ ) (in units of $e^{2} R_{\square} / 2 \pi^{2} \hbar$ ) which determines the temperature cutoff below which EEI is remarkably starts to dominate the temperature dependence of conductivity/resistivity. For all our sample, $T_{0} \approx$ $13 K$ in good agreement with the temperature in which we found that the main contribution to dephasing is the electron electron scattering. Similarly, magnetic field cutoff of WAL effect can be determined from the coherence lengths we extracted in perpendicular transport in the previous section. In order to investigate EEI in the temperature range of $T \leq 13 \mathrm{~K}$, magnetic field cutoff of WAL must be determined at a similar temperature. Therefore, the average values for $L_{\phi b}$ and $L_{\phi s}$ at $T=20$ $\mathrm{K}$ were calculated from the data seen in Fig. 6.6a-e. The approximate values were 80 and $22 \mathrm{~nm}$, for $L_{\phi b}$ and $L_{\phi s}$, respectively. So, the magnetic field cutoffs which are related to phase coherence lengths via $B_{\phi}=\hbar /\left(4 e L_{\phi}^{2}\right)$ were calculated as, $B_{\phi b} \approx 0.03$ $\mathrm{T}$ and $B_{\phi s} \approx 0.34 \mathrm{~T}$ for the bulk and surface channels, respectively. Our investigation of the low temperature behavior of conductivity covered in the larger field range of 
$B \leq 1 \mathrm{~T}$. When the field cutoff is exceeded, the WAL is suppressed and EEI is the only mechanism affecting high field slope values, $|A|_{\text {high }}$. The main mechanism behind this is the fact that magnetic field should not effect the electron-electron Coulomb interaction in the diffusive regime [113]. On the other hand, there are two contributions for $|A|_{\text {low }}$ at zero fields. The first contribution is due to WAL with $\alpha=-0.5$ (per channel), and the second contribution is due to interaction (or EEI) with $\alpha=1$ (per channel). The interaction part with a slope $|A|_{\text {high }} \approx 1$ is the singlet contribution in the diffusion channel. It must be noted that this interaction part also has a triplet contribution in the diffusion channel which is proportional to $\widetilde{F}$.

Figure 6.8 summarizes temperature dependence of conductance with the corresponding fits to the equation (6.4). Despite the original theory estimates a slope $|A|_{\text {low }} \approx 0.5$ (per channel) in zero field, our data indicated a higher value $|A|^{\text {low }} \approx 1.15$ for 12 and 16 QL films confirming the validity of our multiple channel model (Fig. 6.8a-d). The three channel dominated transport must yield $|A|_{\text {low }}=3(-0.5+1)=1.5$ for the slopes at zero fields for our films. However, we observed a slope of 1.15 for 12 , and 16 QL and similarly a slope of 0.65 for 20 , and 25 QL films, which clearly indicated a contribution of two/three channels for the thinnest (Fig. 6.8a-d) and a single channel in thicker films (Fig. 6.8e-h). As the thickness increases, the EEI from surface channel becomes less pronounced and the bulk channel becomes increasingly dominant. With increasing field the slopes must reach 3, at least for the thinnest films which must be the case for 3 independent channels. In fact, for the 12 QL film, slope $|A|_{\text {high }}$ completely saturated 1.72 at a magnetic field of $\mu_{0} H=5 \mathrm{~T}$, indicating a closer to the two channel prediction rather than three channels. The discrepancy between our three channel model WAL and two channel EEI in the thinnest films may result from asymmetric contribution from top and bottom surface states. 

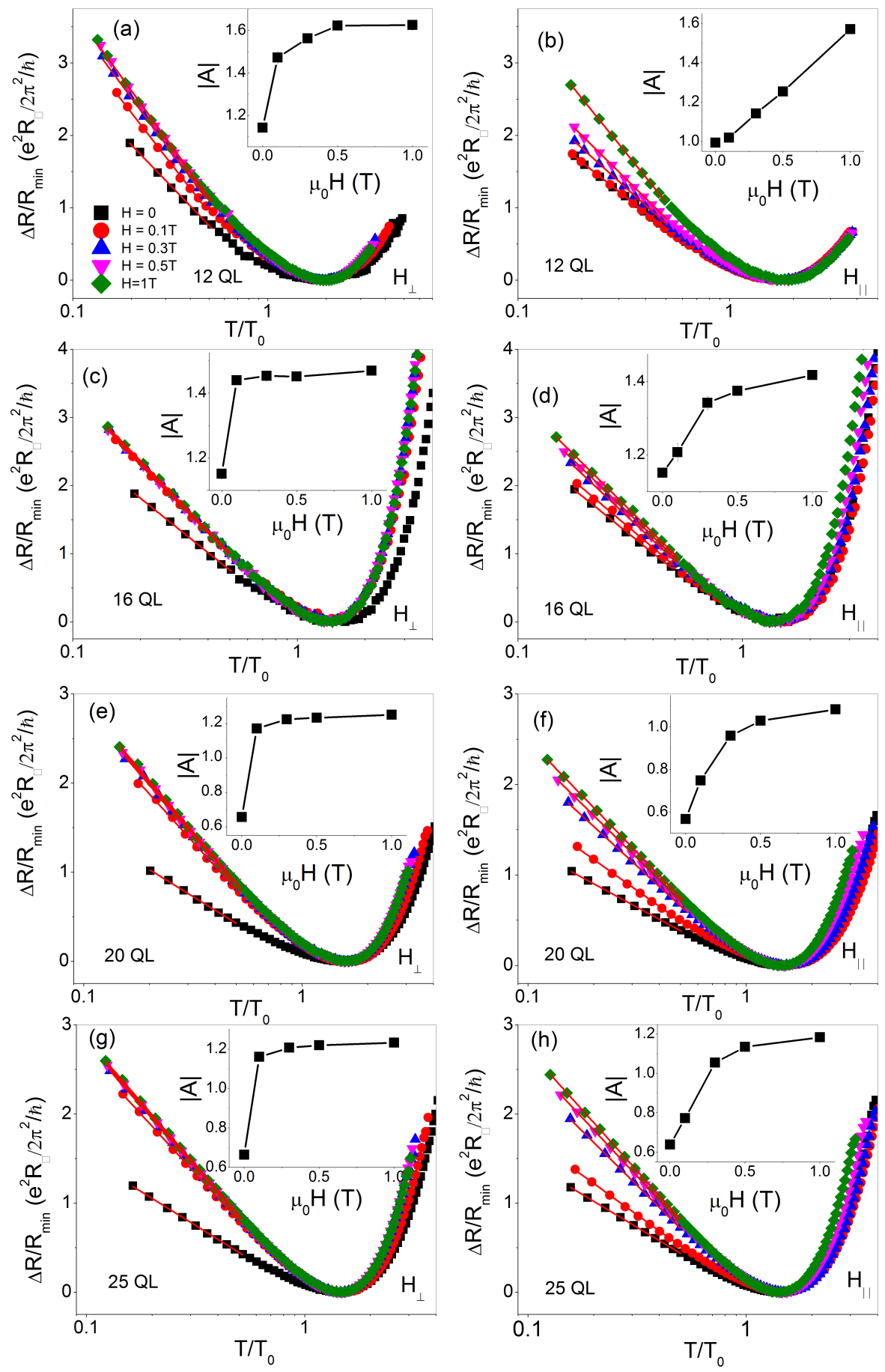

Figure 6.8: Temperature dependence of resistivity in units of $e^{2} R_{\square} / 2 \pi^{2} \hbar$ for different film thickness. Solid red lines represents the fits to equation (6.4) Insets show the slopes $|A|$ determined from $\ln (\mathrm{T})$ dependence. Both perpendicular (left) and parallel (right) field measurements are shown for 12 (a,b), 16 (c,d) 20 (e,f), 25 (g,h) QLs samples, respectively. 
The bulk dominant EEI with increasing thickness is consistent with the thickness dependence of bulk transport and the temperature dependence of bulk phase coherence length. We observed an increase in bulk phase coherence length due to the decrease in EEI in the thicker films. This effect, yielded a slope of 0.65 , and 0.66 for 20 and 25 QLs samples which saturated around 1.25 and 1.23, respectively (insets to Fig. 6.8e-h). These values agree very well with the prediction in the original theory for which the change in slope, $|\Delta A|=|A|_{\text {high }}-|A|_{\text {low }} \approx 0.5$ indicates a single channel. In order to analyze the observed two channel EEI, we must note that the observed behavior can not be a response from two surfaces as the bulk states is also quasi-2D in nature, below $T \leq 13 \mathrm{~K}$. Therefore a bulk contribution to EEI from the bulk channel must exist similarly to the field dependence of magnetoconductance. In order to understand the effects on EEI in bulk and surface states, we systematically investigated the EEI effect in the parallel fields as shown in Fig. 6.8(right). Although the high field slopes must be the same in both field orientations, the data demonstrate that the parallel field slopes do not saturate rapidly in the low field regime. The $12 \mathrm{QL}$ sample shows that the EEI between electrons in the surfaces are very strong and saturates at higher field, $\mu_{0} H \approx 5 \mathrm{~T}$.

I now discuss the behavior of the the screening parameter $\widetilde{F}$. Typically, the slope is expressed by $|A|=(1-\widetilde{F}-p / 2)$ [120], as mentioned earlier. When the quantum interference part (WAL) is quenched, $(p=0)$ then the expression reduces to $|A|=(1-\widetilde{F})$, which is the correction due to the EEI as a result of the diffusive transport for each channel. Including two surface and the bulk states, the slope can be written as $|A|=2\left(1-\widetilde{F}_{s}\right)+\left(1-\widetilde{F}_{b}\right)$, which simplifies to $|A|=3-2 \widetilde{F}_{s}-\widetilde{F}_{b}$. We expect screening parameter for the surface channels, $\widetilde{F}_{s}$ to be the same for all the samples, since the surface contributions must not change with the thickness. By 
using the $12 \mathrm{QL}$ as a reference, the changes in $\widetilde{F}_{b}, \Delta \widetilde{F}_{b}(d)=\widetilde{F}_{b}(d)-\widetilde{F}_{b}(12 \mathrm{QL})$, with respect to the $\widetilde{F}_{b}$ for $12 \mathrm{QL}$ were calculated. Figure 6.9 represents the results for relative change in bulk screening parameters for perpendicular and parallel field measurements. There is a clear increase of trend in $\Delta \widetilde{F}_{b}$ with increasing $d$, which confirms the decreasing EEI with increasing thickness. In other words, decreasing thickness results in less effective screening and an enhanced EEI between electrons in the thinnest films.

$\widetilde{F}$ is one of the most fundamental parameters in 2D EEI theory which is very challenging to estimate theoretically due to the difficulty in calculating many body interactions between electrons [121]. In the modified EEI theories, $\widetilde{F}$ is usually replaced by $F$, which is dimensionless screened Coulomb potential between electrons averaged over the Fermi surface $[119,122]$. The relation between the two parameters is

given by, $\widetilde{F}=(32 / 3 F)\left[(1+F / 2)^{3 / 2}-1-3 F / 4\right][121-123]$. However we must note that the difference between $\widetilde{F}$ and $F$ is only approximately $10 \%$ and negligible in most of the experiments. Addressing the EEI and its effect on the low temperature resistance anomaly requires a more complete understanding of electron electron-electron and electron-impurity interactions in the diffusion channel.

\subsection{Summary and Conclusions}

The field and temperature dependence of conductivity in four high quality $\mathrm{Bi}_{2} \mathrm{Se}_{3}$ thin films was studied in order to provide a more thorough understanding of the transport properties of TIs. The Hall effect data indicated that all the films possesses ntype conductivity due to the electron-like carriers (Se vacancies and antisite defects) in $\mathrm{Bi}_{2} \mathrm{Se}_{3}$. Using a multi-channel version of the Hikami-Larkin-Nagaoka formalism of perpendicular magnetoconductance was used to determine the dependence of the 


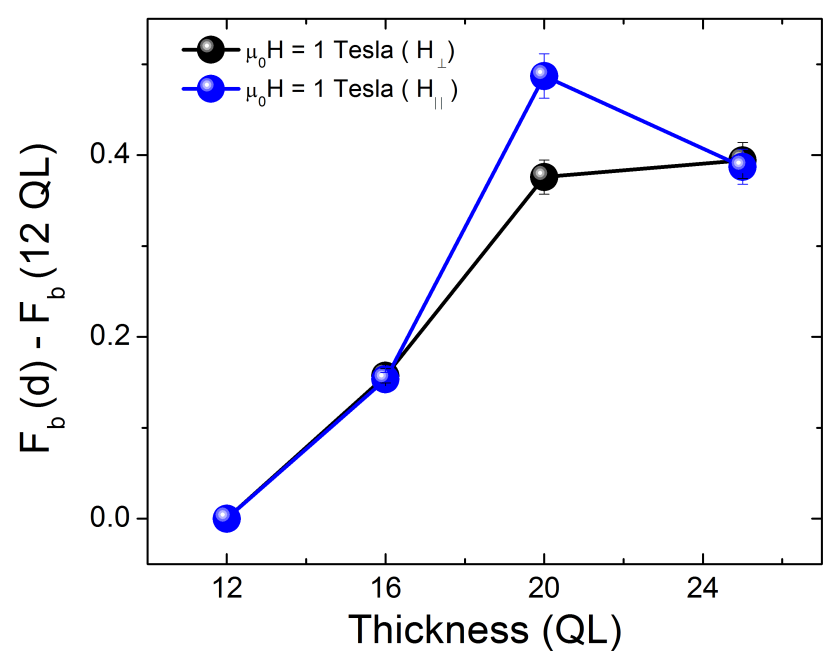

Figure 6.9: The change in dynamically screened Coulomb interaction parameter $\widetilde{F}_{b}$ for the bulk channel as a function of film thickness at $\mu_{o} H=1 \mathrm{~T}$. 12 QL sample was used as a reference to estimate the relative changes in $\widetilde{F}_{b}$ for thicker films.

phase coherence lengths of the bulk and surface electronic states. The coherence lengths of the bulk states was strongly dependent on thickness, while the coherence length of the surface states was independent of thickness as expected. Data from other studies agree with this result. Therefore, this methodology provides a way of disentangling the surface from the bulk conduction, and also shows that at $2 \mathrm{~K}$ the coherence length of the bulk states is approximately $42 \mathrm{~nm}$. Magnetoconductance measurements with the magnetic field applied in the plane of the sample, which are only sensitive to the bulk state conduction, confirmed the results and showed that for the thicker samples, the coherence length was similar in the parallel and perpendicular configurations. For the thinnest (12 QL) sample, there was a disagreement between the two measurements that could be attributed to interactions between the surface states on opposite surfaces of the sample.

The temperature evolution of bulk phase coherence length, indicated that electronelectron scattering was the main source of dephasing. The thickness dependence of 
the dynamically screened Coulomb interaction parameter for the bulk channels was also deduced from the data. The three channel transport model discussed here captures the physics of both field and the temperature dependence of conductivity, if EEI is affected by the electron-impurity interactions [124]. This is also consistent with the structural data which indicated an increase in disorder/impurity level with increasing film thickness. 


\section{Chapter 7}

\section{Summary}

Molecular beam epitaxy was used to grow high quality $\mathrm{Bi}_{2} \mathrm{Se}_{3}$ and $\mathrm{Bi}_{2-x} \mathrm{Mn}_{x} \mathrm{Se}_{3}$ thin films on $\mathrm{Al}_{2} \mathrm{O}_{3}$ single crystal substrates. The growth was implemented in two steps with a substrate temperature of $T_{s}=140{ }^{\circ} \mathrm{C}$ for the first three quintuple layers and, $T_{s}=275{ }^{\circ} \mathrm{C}$ for the remaining layers of the film. Surface quality of the films was monitored in-situ with RHEED which confirmed the epitaxial growth for the

films. Structural quality was investigated using XRD scans. The film thickness and roughness were confirmed from the XRR data and the corresponding fits. Structural data indicated that the films were oriented along the hexagonal c-axis. Rocking curve scans and XRR data analysis confirmed that the roughness/disorder increases in thicker films, for pure $\mathrm{Bi}_{2} \mathrm{Se}_{3}$ thin films. Similar scans and measurements confirmed that the disorder increases with the increasing $\mathrm{Mn}$ doping for $\mathrm{Bi}_{2-x} \mathrm{Mn}_{x} \mathrm{Se}_{3}$ thin films. Electrical contacts were made using silver paste cured at room temperature. For the transport measurements, Hall bar devices with six contacts were patterned with conventional photolithography techniques. The dimensions of Hall bar was $l \times w=1.37 \times 0.50 \mathrm{~mm}$.

EXAFS confirmed that the Mn atoms predominantly occupy the Bi sites in the 
crystal lattice of $\mathrm{Bi}_{2} \mathrm{Se}_{3}$. A valency of $2+$ was obtained for Mn from the XPS analysis. The acceptor nature of $\mathrm{Mn}^{2+}$ was confirmed with the transport measurements.

Unfortunately, the electrical conductivity was dominated by the bulk states in both perpendicular and parallel field measurements. Using a modified model for HLN equation and by considering three independent conducting channels, we extracted the contributions to the magnetoconductance coming from the surface and bulk states independently. Parallel field transport was studied by Altshuler-Aronov formalism which confirmed significant bulk contribution in the transport. The low temperature behavior of conductivity was dominated by the electron-electron interactions.

Future work will focus on improving the $\mathrm{MBE}$ growth of $\mathrm{Bi}_{2} \mathrm{Se}_{3}$ and finding experimental procedures to achieve an insulating bulk for the films. Using the gating techniques might be helpful to deplete the carries in the bulk and allow probing the surface states in transport measurements. Also, smaller sizes of Hall bars need to be patterned to see whether the absence of Shubnikov de Haas oscillations is related to size effects, or to scattering from the edge/boundary of the large Hall bar. 


\section{Bibliography}

[1] Fu, L. and Kane, C.L., Topological insulators with inversion symmetry, Physical Review B, 76(4) (2007).

[2] Hasan, M.Z. and Kane, C.L., Colloquium: Topological insulators, Reviews of Modern Physics, 82(4):3045-3067 (2010).

[3] Moore, J.E., The birth of topological insulators, Nature, 464(7286):194-198 (2010).

[4] Bernevig, B.A., Hughes, T.L., and Zhang, S.C., Quantum spin Hall effect and topological phase transition in HgTe quantum wells, Science, 314(5806):17571761 (2006).

[5] Koenig, M., Wiedmann, S., Bruene, C., Roth, A., Buhmann, H., Molenkamp, L.W., Qi, X.L., and Zhang, S.C., Quantum spin hall insulator state in HgTe quantum wells, Science, 318(5851):766-770 (2007).

[6] Hsieh, D., Qian, D., Wray, L., Xia, Y., Hor, Y.S., Cava, R.J., and Hasan, M.Z., A topological Dirac insulator in a quantum spin Hall phase, Nature, 452(7190):970-U5 (2008).

[7] Xia, Y., Qian, D., Hsieh, D., Wray, L., Pal, A., Lin, H., Bansil, A., Grauer, D., Hor, Y.S., Cava, R.J., and Hasan, M.Z., Observation of a large-gap topological-insulator class with a single Dirac cone on the surface, Nature Physics, 5(6):398-402 (2009).

[8] Qi, X.L. and Zhang, S.C., The quantum spin Hall effect and topological insulators, Physics Today, 63(1):33-38 (2010).

[9] Yu, R., Zhang, W., Zhang, H.J., Zhang, S.C., Dai, X., and Fang, Z., Quantized anomalous Hall effect in magnetic topological insulators, Science, 329(5987):61-64 (2010).

[10] Fu, L. and Kane, C.L., Probing neutral Majorana fermion edge modes with charge transport, Physical Review Letters, 102(21) (2009). 
[11] Tse, W.K. and MacDonald, A.H., Giant Magneto-optical Kerr effect and universal Faraday effect in thin-film topological insulators, Physical Review Letters, 105(5) (2010).

[12] http://jqi.umd.edu/glossary/quantum-hall-effect-and-topological insulators.

[13] Vonklitzing, K., Dorda, G., and Pepper, M., New method for high-accuracy determination of the fine-structure constant based on quantized Hall resistance, Physical Review Letters, 45(6):494-497 (1980).

[14] Kane, C. and Mele, E., Z(2) topological order and the quantum spin Hall effect, Physical Review Letters, 95(14) (2005).

[15] Kane, C. and Mele, E., Quantum spin Hall effect in graphene, Physical Review Letters, 95(22) (2005).

[16] Bernevig, B. and Zhang, S., Quantum spin hall effect, Physical Review Letters, 96(10) (2006).

[17] Zahid, F. and Lake, R., Thermoelectric properties of $\mathrm{Bi}_{2} \mathrm{Te}_{3}$ atomic quintuple thin films, Applied Physics Letters, 97(21) (2010).

[18] Sun, Y., Cheng, H., Gao, S., Liu, Q., Sun, Z., Xiao, C., Wu, C., Wei, S., and Xie, Y., Atomically Thick Bismuth Selenide Freestanding Single Layers Achieving Enhanced Thermoelectric Energy Harvesting, Journal of the American Chemical Society, 134(50):20,294-20,297 (2012).

[19] Hsieh, D., Xia, Y., Qian, D., Wray, L., Dil, J.H., Meier, F., Osterwalder, J., Patthey, L., Checkelsky, J.G., Ong, N.P., Fedorov, A.V., Lin, H., Bansil, A., Grauer, D., Hor, Y.S., Cava, R.J., and Hasan, M.Z., A tunable topological insulator in the spin helical Dirac transport regime, NATURE, 460(7259):1101U59 (2009).

[20] Chen, Y.L., Analytis, J.G., Chu, J.H., Liu, Z.K., Mo, S.K., Qi, X.L., Zhang, H.J., Lu, D.H., Dai, X., Fang, Z., Zhang, S.C., Fisher, I.R., Hussain, Z., and Shen, Z.X., Experimental realization of a three-dimensional topological insulator, $\mathrm{Bi}_{2} \mathrm{Te}_{3}$, Science, 325(5937):178-181 (2009).

[21] Nakahara, M., Geometry, Topology and Physics, Institute of Physics Publishing (1990).

[22] Shen, S.Q., Topological Insulators, Springer (2012).

[23] Lu, H.Z., Shi, J., and Shen, S.Q., Competition between weak localization and antilocalization in topological surface states, Physical Review Letters, 107(7) (2011). 
[24] Hikami, S., Larkin, A., and Nagaoka, Y., Spin-orbit interaction and magnetoresistance in the 2 dimensional random system, Progress of Theoretical Physics, 63(2):707-710 (1980).

[25] Altshuler, B., Aronov, A., and Khmelnitsky, D., Effects of electron electron collisions with small energy transfers on quantum localization, Journal of Physics C-Solid State Physics, 15(36):7367-7386 (1982).

[26] Gershenson, M., Low-temperature dephasing in disordered conductors: experimental aspects, Annalen Der Physik, 8(7-9):559-568 (1999).

[27] Bergmann, G., Weak localization in thin films - A time-of-flight experiment with conduction electrons, Physics Reports-Review Section of Physics Letters, 107(1):1-58 (1984).

[28] Lee, P. and Ramakrishnan, T., Disordered electronic systems, Reviews of Modern Physics, 57(2):287-337 (1985).

[29] Eaglesham, D., Semiconductor molecular-beam epitaxy at low-temperatures, Journal of Applied Physics, 77(8):3597-3617 (1995).

[30] Harbison, J., Sands, T., Tabatabaie, N., Chan, W., Florez, L., and Keramidas, V., Molecular-beam epitaxial-growth of ultrathin buried metal layers - (Al,Ga)As/NiAl/(Al,Ga)As heterostructures, Applied Physics Letters, 53(18):1717-1719 (1988).

[31] Ploog, K., Fischer, A., Trommer, R., and Hirose, M., MBE-grown insulating oxide-films on GaAs, Journal of Vacuum Science \& Technology, 16(2):290-294 (1979).

[32] Herman, M., Molecular beam epitaxy : fundamentals and current status, Springer-Verlag, Berlin New York (1989).

[33] Yamamoto, Y., Reflection high-energy electron-diffraction study of superstructures induced on a $\mathrm{Si}(110)$ surface by adsorption, Japanese Journal of Applied Physics Part 2-Letters, 31(1A-B):L53-L56 (1992).

[34] Svensson, S., Nilsson, P., and Andersson, T., Interpretation of low-energy Auger-spectra from AlAs and $\mathrm{GaAs}(001)$ - applications to surface preparation, Physical Review B, 31(8):5272-5279 (1985).

[35] Maracas, G., Edwards, J., Shiralagi, K., Choi, K., Droopad, R., Johs, B., and Woolam, J., Insitu spectroscopic ellipsometry in molecular-beam-epitaxy, Journal of Vacuum Science \&3 Technology A-Vacuum Surfaces and Films, 10(4, 2):1832-1839 (1992). 
[36] Sitter, H., Glanner, G., and Herman, M., Exact determination of the real substrate-temperature and film thickness in vacuum epitaxial-growth systems by visible laser interferometry, Vacuum, 46(1):69-76 (1995).

[37] http://www.lps.umd.edu/MBEGroup/MBEWhatIs.htm. (2014).

[38] http://www.ece.gatech.edu/research/labs/vc/theory/photolith.html (2014).

[39] Dobson, P., Joyce, B., Neave, J., and Zhang, J., Current understanding and applications of the RHEED Intensity oscillation technique, Journal of Crystal Growth, 81(1-4):1-8 (1987).

[40] Neave, J., JoyceE, B., Dobson, P., and Norton, N., Dynamics of film growth of GaAs by MBE From RHEED Observations, Applied Physics A-Materials Science $\mathscr{E}$ Processing, 31(1):1-8 (1983).

[41] Zhang, G., Qin, H., Teng, J., Guo, J., Guo, Q., Dai, X., Fang, Z., and Wu, K., Quintuple-layer epitaxy of thin films of topological insulator $\mathrm{Bi}_{2} \mathrm{Se}_{3}$, Applied Physics Letters, 95(5) (2009).

[42] Warren, B.E., X-ray diffraction, Dover Publications, New York (1990).

[43] Myers, H.P., Introductory solid state physics, Taylor \& Francis, London (1997).

[44] Giacovazzo, C., Fundamentals of crystallography, Oxford University Press, Oxford New York (2011).

[45] Jensen, J., Oelkers, A., Toivola, R., Johnson, D., Elam, J., and George, S., Xray reflectivity characterization of $\mathrm{ZnO} / \mathrm{Al}_{2} \mathrm{O}_{3}$ multilayers prepared by atomic layer deposition, Chemistry of Materials, 14(5):2276-2282 (2002).

[46] Fullerton, E., Schuller, I., Vanderstraeten, H., and Bruynseraede, Y., Structural refinement of superlattices from x-ray-diffraction, Physical Review B, 45(16):9292-9310 (1992).

[47] Gibaud, A. and Hazra, S., X-ray reflectivity and diffuse scattering, Current Science, 78(12):1467-1477 (2000).

[48] Parratt, L., Surface studies of solids by total reflection of x-rays, Physical Review, 95(2):359-369 (1954).

[49] Nevot, L. and Croce, P., Characterization of surfaces by grazing x-ray reflection - Application to study of polishing of some silicate-glasses, Revue de Physique Appliquee, 15(3):761-779 (1980).

[50] J., T., Scanning probe microscopy training notebook, Veeco (2000). 
[51] Teo, B., EXAFS: Basic principles and data analysis, Springer Berlin Heidelberg, Berlin, Heidelberg (1986).

[52] Prochazka, V., Study of cobaltites and manganites by NMR and EXAFS, Ph.D. thesis, Charles University in Prague (2009).

[53] Ashcroft, N., Solid state physics, Holt, Rinehart and Winston, New York (1976).

[54] Bansal, N., Kim, Y.S., Brahlek, M., Edrey, E., and Oh, S., Thicknessindependent transport channels in topological insulator $\mathrm{Bi}_{2} \mathrm{Se}_{3}$ thin films, Physical Review Letters, 109(11) (2012).

[55] Tabor, P., Keenan, C., Urazdhin, S., and Lederman, D., Molecular beam epitaxy and characterization of thin $\mathrm{Bi}_{2} \mathrm{Se}_{3}$ films on $\mathrm{AL}_{2} \mathrm{O}_{3}$ (110), Applied Physics Letters, 99(1) (2011).

[56] Glinka, Y.D., Babakiray, S., Johnson, T.A., Bristow, A.D., Holcomb, M.B., and Lederman, D., Ultrafast carrier dynamics in thin-films of the topological insulator $\mathrm{Bi}_{2} \mathrm{Se}_{3}$, Applied Physics Letters, 103(15) (2013).

[57] Wang, Z., Lin, T., Wei, P., Liu, X., Dumas, R., Liu, K., and Shi, J., Tuning carrier type and density in $\mathrm{Bi}_{2} \mathrm{Se}_{3}$ by Ca-doping, Applied Physics Letters, 97(4) (2010).

[58] Schreyeck, S., Tarakina, N.V., Karczewski, G., Schumacher, C., Borzenko, T., Bruene, C., Buhmann, H., Gould, C., Brunner, K., and Molenkamp, L.W., Molecular beam epitaxy of high structural quality $\mathrm{Bi}_{2} \mathrm{Se}_{3}$ on lattice matched InP(111) substrates, Applied Physics Letters, 102(4) (2013).

[59] Bjorck, M. and Andersson, G., GenX: an extensible x-ray reflectivity refinement program utilizing differential evolution, Journal of Applied Crystallography, 40(6):1174-1178 (2007).

[60] Munbodh, K., Cheon, M., Lederman, D., Fitzsimmons, M.R., and Dilley, N.R., Interfacial coupling between ferromagnets and random and dilute antiferromagnets, Physical Review B, 84(21) (2011).

[61] Marcus, M., MacDowell, A., Celestre, R., Manceau, A., Miller, T., Padmore, H., and Sublett, R., Beamline 10.3.2 at ALS: a hard X-ray microprobe for environmental and materials sciences, Journal of Synchrotron Radiation, 11(3):239-247 (2004).

[62] Liu, M., Zhang, J., Chang, C.Z., Zhang, Z., Feng, X., Li, K., He, K., Wang, L.L., Chen, X., Dai, X., Fang, Z., Xue, Q.K., Ma, X., and Wang, Y., Crossover 
between Weak Antilocalization and Weak Localization in a Magnetically Doped Topological Insulator, Physical Review Letters, 108(3) (2012).

[63] Cha, J.J., Claassen, M., Kong, D., Hong, S.S., Koski, K.J., Qi, X.L., and Cui, Y., Effects of Magnetic Doping on Weak Antilocalization in Narrow $\mathrm{Bi}_{2} \mathrm{Se}_{3}$ Nanoribbons, Nano Letters, 12(8):4355-4359 (2012).

[64] Haazen, P.P.J., Laloe, J.B., Nummy, T.J., Swagten, H.J.M., Jarillo-Herrero, P., Heiman, D., and Moodera, J.S., Ferromagnetism in thin-film Cr-doped topological insulator $\mathrm{Bi}_{2} \mathrm{Se}_{3}$, Applied Physics Letters, 100(8) (2012).

[65] Zhang, J.M., Zhu, W., Zhang, Y., Xiao, D., and Yao, Y., Tailoring Magnetic Doping in the Topological Insulator $\mathrm{Bi}_{2} \mathrm{Se}_{3}$, Physical Review Letters, 109(26) (2012).

[66] Zhang, D., Richardella, A., Rench, D.W., Xu, S.Y., Kandala, A., Flanagan, T.C., Beidenkopf, H., Yeats, A.L., Buckley, B.B., Klimov, P.V., Awschalom, D.D., Yazdani, A., Schiffer, P., Hasan, M.Z., and Samarth, N., Interplay between ferromagnetism, surface states, and quantum corrections in a magnetically doped topological insulator, Physical Review B, 86(20) (2012).

[67] Choi, Y.H., Jo, N.H., Lee, K.J., Lee, H.W., Jo, Y.H., Kajino, J., Takabatake, T., Ko, K.T., Park, J.H., and Jung, M.H., Simple tuning of carrier type in topological insulator $\mathrm{Bi}_{2} \mathrm{Se}_{3}$ by Mn doping, Applied Physics Letters, 101(15) (2012).

[68] Babakiray, S., Johnson, T.A., Borisov, P., Holcomb, M.B., Lederman, D., Marcus, M.A., and Tarafder, K., Structural properties of $\mathrm{Bi}_{2-x} \mathrm{Mn}_{x} \mathrm{Se}_{3}$ thin films grown via molecular beam epitaxy, Journal of Applied Physics, 118(4):045302 (2015).

[69] Hagmann, J.A., Magnetotransport investigation of bismuth chalcogenide topological insulators,, Ph.D. thesis, University of Notre Dame, Notre Dame,Indiana, USA (2013).

[70] Mandale, A., Badrinarayanan, S., Date, S., and Sinha, A., Photoelectronspectroscopic study of nickel, manganese and cobalt selenides, Journal of Electron Spectroscopy and Related Phenomena, 33(1):61-72 (1984).

[71] Kong, D., Cha, J.J., Lai, K., Peng, H., Analytis, J.G., Meister, S., Chen, Y., Zhang, H.J., Fisher, I.R., Shen, Z.X., and Cui, Y., Rapid surface oxidation as a source of surface degradation factor for $\mathrm{Bi}_{2} \mathrm{Se}_{3}$, Acs Nano, 5(6):4698-4703 (2011). 
[72] Golyashov, V.A., Kokh, K.A., Makarenko, S.V., Romanyuk, K.N., Prosvirin, I.P., Kalinkin, A.V., Tereshchenko, O.E., Kozhukhov, A.S., Sheglov, D.V., Eremeev, S.V., Borisova, S.D., and Chulkov, E.V., Inertness and degradation of (0001) surface of $\mathrm{Bi}_{2} \mathrm{Se}_{3}$ topological insulator, Journal of Applied Physics, 112(11) (2012).

[73] de Groot, F. and Kotani, A., Core level spectroscopy of solids introduction, in Core Level Spectroscopy of Solids, volume 6 of Advances in Condensed Matter Science, pp. 1+ (2008).

[74] Langell, M., Hutchings, C., Carson, G., and Nassir, M., High resolution electron energy loss spectroscopy of $\mathrm{MnO}(100)$ and oxidized $\mathrm{MnO}(100)$, Journal of Vacuum Science 83 Technology A-Vacuum Surfaces and Films, 14(3, 2):16561661 (1996).

[75] Stranick, A., $\mathrm{Mn}_{2} \mathrm{O}_{3}$ by XPS, Surface Science Spectra, 6:39 (1999).

[76] Iwanowski, R., Heinonen, M., and Janik, E., X-ray photoelectron spectra of zinc-blende MnTe, Chemical Physics Letters, 387(1-3):110-115 (2004).

[77] Vanderheide, H., Hemmel, R., and Vanbruggen, C., X-ray photoelectronspectra of 3d transition-metal pyrites, Journal of Solid State Chemistry, 33(1):17-25 (1980).

[78] Figueroa, A.I., van der Laan, G., Collins-McIntyre, L.J., Zhang, S.L., Baker, A.A., Harrison, S.E., Schoenherr, P., Cibin, G., and Hesjedal, T., Magnetic Cr doping of $\mathrm{Bi}_{2} \mathrm{Se}_{3}$ : Evidence for divalent $\mathrm{Cr}$ from x-ray spectroscopy, Physical Review B, 90(13) (2014).

[79] Ravel, B. and Newville, M., Athena, Artemis, Hephaestus: data analysis for Xray absorption spectroscopy using IFEFFIT, Journal of Synchrotron Radiation, 12(4):537-541 (2005).

[80] Nakajima, S., Crystal structure of $\mathrm{Bi}_{2} \mathrm{Te}_{3-x} \mathrm{Se}_{x}$, Journal of Physics and Chemistry of Solids, 24(3):479-\& (1963).

[81] Brode, R., The quantitative study of the collisions of electrons with atoms, Reviews of Modern Physics, 5(4):0257-0279 (1933).

[82] d'Acapito, F., Smolentsev, G., Boscherini, F., Piccin, M., Bais, G., Rubini, S., Martelli, F., and Franciosi, A., Site of Mn in Mn delta-doped GaAs: X-ray absorption spectroscopy, Physical Review B, 73(3) (2006).

[83] Liu, Z., Wei, X., Wang, J., Pan, H., Ji, F., Xi, F., Zhang, J., Hu, T., Zhang, S., Jiang, Z., Wen, W., Huang, Y., Ye, M., Yang, Z., and Qiao, S., Local 
structures around $3 \mathrm{~d}$ metal dopants in topological insulator $\mathrm{Bi}_{2} \mathrm{Se}_{3}$ studied by EXAFS measurements, Physical Review B, 90(9) (2014).

[84] Kresse, G. and Hafner, J., Ab initio molecular dynamics for liquid metals, Physical Review B, 47:558-561 (1993).

[85] Kresse, G. and Furthmüller, J., Efficient iterative schemes for ab initio totalenergy calculations using a plane-wave basis set, Physical Review B, 54:11,169 11,186 (1996).

[86] Momma, K. and Izumi, F., VESTA 3 for three-dimensional visualization of crystal, volumetric and morphology data, Journal of Applied Crystallography, 44(6):1272-1276 (2011).

[87] Abdalla, L.B., Seixas, L., Schmidt, T.M., Miwa, R.H., and Fazzio, A., Topological insulator $\mathrm{Bi}_{2} \mathrm{Se}_{3}(111)$ surface doped with transition metals: An ab initio investigation, Physical Review B, 88:045,312 (2013).

[88] Jacobson, A.J. and Fender, B.E.F., Covalency parameters in MnSe and $\mathrm{MnSe}_{2}$, The Journal of Chemical Physics, 52(9):4563-4566 (1970).

[89] C. P. Gazzara, R. M. Middleton, R.J.W. and Hall, E.O., A refinement of the parameters of $\alpha$ manganese, Acta Cryst., 22:859-862 (1967).

[90] Hor, Y.S., Williams, A.J., Checkelsky, J.G., Roushan, P., Seo, J., Xu, Q., Zandbergen, H.W., Yazdani, A., Ong, N.P., and Cava, R.J., Superconductivity in $\mathrm{Cu}_{x} \mathrm{Bi}_{2} \mathrm{Se}_{3}$ and its implications for pairing in the undoped topological insulator, Physical Review Letters, 104(5) (2010).

[91] Brahlek, M., Koirala, N., Salehi, M., Bansal, N., and Oh, S., Emergence of decoupled surface transport channels in bulk insulating $\mathrm{Bi}_{2} \mathrm{Se}_{3}$ thin films, Physical Review Letters, 113(2) (2014).

[92] Babakiray, S., Johnson, T.A., Borisov, P., Holcomb, M.B., Lederman, D., Marcus, M.A., and Tarafder, K., Structural properties of $\mathrm{Bi}_{2-x} \mathrm{Mn}_{x} \mathrm{Se}_{3}$ thin films grown via molecular beam epitaxy, Journal of Applied Physics, 118(4) (2015).

[93] Liu, M., Chang, C.Z., Zhang, Z., Zhang, Y., Ruan, W., He, K., Wang, L.l., Chen, X., Jia, J.F., Zhang, S.C., Xue, Q.K., Ma, X., and Wang, Y., Electron interaction-driven insulating ground state in $\mathrm{Bi}_{2} \mathrm{Se}_{3}$ topological insulators in the two-dimensional limit, Physical Review B, 83(16) (2011).

[94] Altshuler, B.L., Aronov, A.G., and Lee, P.A., Interaction effects in disordered Fermi systems in two dimensions, Physical Review Letters, 44:1288-1291 (1980). 
[95] Hor, Y.S., Richardella, A., Roushan, P., Xia, Y., Checkelsky, J.G., Yazdani, A., Hasan, M.Z., Ong, N.P., and Cava, R.J., P-type $\mathrm{Bi}_{2} \mathrm{Se}_{3}$ for topological insulator and low-temperature thermoelectric applications, Physical Review B, 79(19) (2009).

[96] Kong, D., Chen, Y., Cha, J.J., Zhang, Q., Analytis, J.G., Lai, K., Liu, Z., Hong, S.S., Koski, K.J., Mo, S.K., Hussain, Z., Fisher, I.R., Shen, Z.X., and Cui, Y., Ambipolar field effect in the ternary topological insulator $\left(\mathrm{Bi}_{x} \mathrm{Sb}_{1-x}\right)_{2} \mathrm{Te}_{3}$ by composition tuning, Nature Nanotechnology, 6(11):705-709 (2011).

[97] Zhang, J., Chang, C.Z., Zhang, Z., Wen, J., Feng, X., Li, K., Liu, M., He, K., Wang, L., Chen, X., Xue, Q.K., Ma, X., and Wang, Y., Band structure engineering in $\left(\mathrm{Bi}_{1-x} \mathrm{Sb}_{x}\right)_{2} \mathrm{Te}_{3}$ ternary topological insulators, Nature Communications, 2 (2011).

[98] Xue, L., Zhou, P., Zhang, C.X., He, C.Y., Hao, G.L., Sun, L.Z., and Zhong, J.X., First-principles study of native point defects in $\mathrm{Bi}_{2} \mathrm{Se}_{3}$, AIP Advances, 3(5) (2013).

[99] Kim, Y.S., Brahlek, M., Bansal, N., Edrey, E., Kapilevich, G.A., Iida, K., Tanimura, M., Horibe, Y., Cheong, S.W., and Oh, S., Thickness-dependent bulk properties and weak antilocalization effect in topological insulator $\mathrm{Bi}_{2} \mathrm{Se}_{3}$ , Physical Review B, 84(7) (2011).

[100] Glinka, Y.D., Babakiray, S., and Lederman, D., Plasmon-enhanced electronphonon coupling in Dirac surface states of the thin-film topological insulator $\mathrm{Bi}_{2} \mathrm{Se}_{3}$, Journal of Applied Physics, 118(13) (2015).

[101] Kondo, J., Resistance minimum in dilute magnetic alloys, Progress of Theoretical Physics, 32(1):37-\& (1964).

[102] Analytis, J.G., Chu, J.H., Chen, Y., Corredor, F., McDonald, R.D., Shen, Z.X., and Fisher, I.R., Bulk Fermi surface coexistence with Dirac surface state in $\mathrm{Bi}_{2} \mathrm{Se}_{3}$ : A comparison of photoemission and Shubnikov-de Haas measurements, Physical Review B, 81(20) (2010).

[103] Glinka, Y.D., Babakiray, S., Johnson, T.A., Holcomb, M.B., and Lederman, D., Effect of carrier recombination on ultrafast carrier dynamics in thin films of the topological insulator $\mathrm{Bi}_{2} \mathrm{Se}_{3}$, Applied Physics Letters, 105(17) (2014).

[104] He, H.T., Wang, G., Zhang, T., Sou, I.K., Wong, G.K.L., Wang, J.N., Lu, H.Z., Shen, S.Q., and Zhang, F.C., Impurity Effect on Weak Antilocalization in the Topological Insulator $\mathrm{Bi}_{2} \mathrm{Te}_{3}$, Physical Review Letters, 106(16) (2011). 
[105] Wu, X., Li, X., Song, Z., Berger, C., and de Heer, W.A., Weak antilocalization in epitaxial graphene: Evidence for chiral electrons, Physical Review Letters, 98(13) (2007).

[106] Wang, H., Liu, H., Chang, C.Z., Zuo, H., Zhao, Y., Sun, Y., Xia, Z., He, K., Ma, X., Xie, X.C., Xue, Q.K., and Wang, J., Crossover between Weak Antilocalization and Weak Localization of Bulk States in Ultrathin $\mathrm{Bi}_{2} \mathrm{Se}_{3}$ Films, Scientific Reports, 4 (2014).

[107] Li, Z., Chen, T., Pan, H., Song, F., Wang, B., Han, J., Qin, Y., Wang, X., Zhang, R., Wan, J., Xing, D., and Wang, G., Two-dimensional universal conductance fluctuations and the electron-phonon interaction of surface states in $\mathrm{Bi}_{2} \mathrm{Te}_{2}$ Se microflakes, Scientific Reports, 2 (2012).

[108] Zhang, Y., He, K., Chang, C.Z., Song, C.L., Wang, L.L., Chen, X., Jia, J.F., Fang, Z., Dai, X., Shan, W.Y., Shen, S.Q., Niu, Q., Qi, X.L., Zhang, S.C., Ma, X.C., and Xue, Q.K., Crossover of the three-dimensional topological insulator Bi2Se3 to the two-dimensional limit (vol 6, pg 584, 2010), Nature Physics, 6(9) (2010).

[109] Lin, C.J., He, X.Y., Liao, J., Wang, X.X., Sacksteder, V., Yang, W.M., Guan, T., Zhang, Q.M., Gu, L., Zhang, G.Y., Zeng, C.G., Dai, X., Wu, K.H., and Li, Y.Q., Parallel field magnetoresistance in topological insulator thin films, Physical Review B, 88(4) (2013).

[110] Kohler, E.W.H., The g factor of the conduction electrons in $\mathrm{Bi}_{2} \mathrm{Se}_{3}$, Phys. Stat. Sol. (b) , 67(665) (1975).

[111] Glinka, Y.D., Babakiray, S., Johnson, T.A., and Lederman, D., Thickness tunable quantum interference between surface phonon and Dirac plasmon states in thin films of the topological insulator $\mathrm{Bi}_{2} \mathrm{Se}_{3}$, Journal of Physics-Condensed Matter, 27(5) (2015).

[112] Cao, H., Xu, S., Miotkowski, I., Tian, J., Pandey, D., Hasan, M.Z., and Chen, Y.P., Structural and electronic properties of highly doped topological insulator $\mathrm{Bi}_{2} \mathrm{Se}_{3}$ crystals, Physica Status Solidi-Rapid Research Letters, 7(1-2, SI):133135 (2013).

[113] M.E. Gershenzon, B.N. Gubankov, Y.E.Z., Effects of "weak" localization and of electron-electron interaction in thin copper and silver films, Sov. Phys. Jetp, 56(6) (1982).

[114] Altshuler, B. and Aronov, A., Magnetoresistance of thin-films and of wires in a longitudinal magnetic-field, Jetp Letters, 33(10):499-501 (1981). 
[115] Glinka, Y.D., Babakiray, S., Johnson, T.A., Holcomb, M.B., and Lederman, D., Resonance-type thickness dependence of optical second-harmonic generation in thin films of the topological insulator $\mathrm{Bi}_{2} \mathrm{Se}_{3}$, Physical Review B, 91(19) (2015).

[116] Glinka, Y.D., Babakiray, S., Johnson, T.A., Holcomb, M.B., and Lederman, D., Acoustic phonon dynamics in thin-films of the topological insulator $\mathrm{Bi}_{2} \mathrm{Se}_{3}$, Journal of Applied Physics, 117(16) (2015).

[117] Bas, D.A., Vargas-Velez, K., Babakiray, S., Johnson, T.A., Borisov, P., Stanescu, T.D., Lederman, D., and Bristow, A.D., Coherent control of injection currents in high-quality films of $\mathrm{Bi}_{2} \mathrm{Se}_{3}$, Applied Physics Letters, 106(4) (2015).

[118] Altshuler, B., Aronov, A., and Lee, P., Interaction Effects in Disordered Fermi Systems in 2 Dimensions, Physical Review Letters, 44(19):1288-1291 (1980).

[119] Takagaki, Y., Jenichen, B., Jahn, U., Ramsteiner, M., and Friedland, K.J., Weak antilocalization and electron-electron interaction effects in $\mathrm{Cu}$-doped $\mathrm{Bi}_{2} \mathrm{Se}_{3}$ films, Physical Review B, 85(11) (2012).

[120] Gershenzon, M.E., G.V. and Zhuravlev, Y., Weak Localization and ElectronScattering in Silver Thin Films, Jetp Letters, 35(11):576-580 (1982).

[121] Lin, J., Hsu, S., Lue, J., and Sheng, P., Spin-orbit scattering effect on electronelectron interactions in disordered metals, Journal of Physics and Chemistry of Solids, 62(9-10):1813-1818 (2001).

[122] Chiu, S.P. and Lin, J.J., Weak antilocalization in topological insulator $\mathrm{Bi}_{2} \mathrm{Te}_{3}$ microflakes, Physical Review B, 87(3) (2013).

[123] Lee, P.A. and Ramakrishnan, T.V., Disordered Electronic Systems, Rev. Mod. Phys., 57:287 (1985).

[124] Reizer, M., Electron-electron interaction effect on the conductivity and the Hall conductivity of weakly disordered electron systems, Physical Review B, 57(19):12,338-12,344 (1998). 